UDC 582.594(597)

\title{
New and rare orchids (Orchidaceae) in the flora of Cambodia and Laos
}

\author{
L. V. Averyanov ${ }^{1}$, Khang Sinh Nguyen ${ }^{2}$, T. V. Maisak ${ }^{1}$, E. L. Konstantinov ${ }^{3}$, \\ Tien Hiep Nguyen ${ }^{4}$, Somchanh Bounphanmy ${ }^{5}$ \\ ${ }^{1}$ Russian Academy of Sciences, Komarov Botanical Institute, Prof. Popov, 2; St. Petersburg, RF-197376, Russia. \\ E-mail:av_leonid@mail.ru; av_leonid@yahoo.com \\ ${ }^{2}$ Institute of Ecology and Biological Resources, Vietnam Academy of Science and Technology, 18 Hoang Quoc Viet, Cau Giay, \\ Ha Noi, Vietnam.E-mail: khangnguyensinh@yahoo.com \\ ${ }^{3}$ Kaluga State University, Kaluga, Stepan Razin str., 26, RF-0248023, Russia. E-mail: nepentes@list.ru \\ ${ }^{4}$ Center for Plant Conservation, No 25/32, lane 191, Lac Long Quan, Nghia Do, Cau Giay District, Ha Noi, Vietnam \\ E-mail: hiep.nguyen@cpcvn.org \\ ${ }^{5}$ National University of Laos, Faculty of Science, Dept of Biology, Dong Dok, PO Box 7322, Vientian, LAO PDR \\ E-mail: sbounphanmy@nuol.edu.la; somchanh@gmail.com
}

Key words: Cambodia, Laos, flora, Orchidaceae, plant diversity.

Summary. Herbarium material collected in 2013-2015 in Cambodia and Laos provides 298 new localities for 167 orchid species (from 57 genera). Almost all reported locations are new on the territory of studied countries. These data provide substantial basis for understanding of present day distribution of rare species needed protection. Among reported species, 8 species are new for the flora of Cambodia (Bulbophyllum macranthum, B. orientale, B. physocoryphum, B. retusiusculum, Dendrobium pachyphyllum, Eria tenuiflora, Pholidota recurva, Thelasis pygmaea) and 43 species are new for the flora of Laos (Bulbophyllum ambrosia, B. andersonii, B. bicolor, B. bisetoides, B. delitescens, B. depressum, B. forrestii, B. lilacinum, B. longibrachiatum, B. psyhoon, Calanthe alismifolia, C. sylvatica, Cheirostylis pusilla, Cleisostoma melanorachis, C. subulatum, C. williamsonii, Coelogyne eberhardtii, C. leucantha, C. ovalis, Dendrobium eriiflorum, D. falconeri, D. trantuanii, D. williamsonii, Didymoplexis pallens, Epigeneium fuscescens, Eria apertiflora, E. bambusifolia, E. pachyphylla, Flickingeria fimbriata, Gastrochilus suavis, Liparis mannii, L. sootenzanensis, Luisia ramosii, Oberonia kwangsiensis, O. maxima, Pelatantheria insectifera, Phalaenopsis braceana, Pholidota yunnanensis, Schoenorchis scolopendria, S. tixieri, Tainia cordifolia, Thrixspermum calceolum). Five species (Dendrobium minusculum, Oberonia simplex, Porpax capuccinorum, P. laosensis and Taeniophyllum filiferum) discovered in Cambodia in Laos are described as new for science. One genus (Didymoplexis) was found in Laos at first. Such rare ornamental species as Aerides falcata, A. Alabellata, A. rosea, Arundina caespitosa, Ascolabium pusillum, Bulbophyllum bicolor, Calanthe cardioglossa, Coelogyne assamica, C. calcicola, C. eberhardtii, C. Alaccida, C. trinervis, Dendrobium aphyllum, D. christyanum, D. chryseum, D. chrysotoxum, D. crepidatum, D. dixanthum, D. falconeri, D. fimbriatum, D. findleyanum, D. gratiosissimum, D. jenkinsii, D. loddigesii, D. nobile, D. senile, D. thyrsiflorum, D. trantuanii, D. trigonopus, D. williamsonii, Doritis pulcherrima, Gastrochilus suavis, Holcoglossum kimballianum, H. subulifolium, Hygrochilus parishii, Paphiopedilum concolor, P. dianthum, Phalaenopsis braceana, Schoenorchis scolopendria, S. tixieri, Spathoglottis affinis, Tainia cordifolia, Vanda brunnea, $V$. denisoniana, $V$. flavobrunnea become presently very rare on the territory of studied local floras and badly need protection to prevent their extinction in nature. 


\title{
Новые и редкие виды орхидных во флоре Камбоджи и Лаоса
}

\author{
Л. В. Аверьянов ${ }^{1}$, Кхан Синь Нгуен ${ }^{2}$, Т. В. Майсак ${ }^{1}$, Е. Л. Константинов ${ }^{3}$, \\ Тьен Хьеп Нгуенн ${ }^{4}$ Сомчан Бонфанами
}

\begin{abstract}
${ }^{I}$ Ботанический институт им. В. Л. Комарова РАН, ул. Проф. Попова, 2; Санкт-Петербург, 197376, Россия
${ }^{2}$ Институт экологи и биологических ресурсов, Академии наук и технологии Вьетнама, 18 Хоанг Куок Миуе, Лай Гау, Ханой, Вьетнам

${ }^{3}$ Калужский государственный университет, Калуга, ул. Степана Разина, 26, RF-0248023, Россия

${ }^{4}$ Центр охраны растений, о 25/32, линия 191, Лак Лонг Куан, Ни До, Кай Гау, Ханой, Вьетнам
\end{abstract}

${ }^{5}$ Национальный университет Лаоса, фокультет науки,отдел биологии, Донг Док, РО 7322, Вьентьян, Лаос

Ключевые слова: Камбоджа, Лаос, флора, орхидные, Orchidaceae, разнообразие растений.

\begin{abstract}
Аннотация. Гербарные сборы 2013-2015 годов документируют 298 новых нахождений 167 видов орхидных (из 57 родов) в Камбодже и Лаосе. Почти все приведенные местонахождения являются новыми для территорий изученных стран. Эти данные существенно расширяют современные знания о распространении редких растений, нуждающихся в охране. Среди приводимых видов 8 являются новыми для флоры Камбоджи (Bulbophyllum macranthum, B. orientale, B. physocoryphum, B. retusiusculum, Dendrobium pachyphyllum, Eria tenuiflora, Pholidota recurva, Thelasis pygmaea) и 43 - новые для флоры Лаоса (Bulbophyllum ambrosia, B. andersonii, B. bicolor, B. bisetoides, B. delitescens, B. depressum, B. forrestii, B. lilacinum, B. longibrachiatum, B. psyhoon, Calanthe alismifolia, C. sylvatica, Cheirostylis pusilla, Cleisostoma melanorachis, C. subulatum, C. williamsonii, Coelogyne eberhardtii, C. leucantha, C. ovalis, Dendrobium eriiflorum, D. falconeri, D. trantuanii, D. williamsonii, Didymoplexis pallens, Epigeneium fuscescens, Eria apertiflora, E. bambusifolia, E. pachyphylla, Flickingeria fimbriata, Gastrochilus suavis, Liparis mannii, L. sootenzanensis, Luisia ramosii, Oberonia kwangsiensis, O. maxima, Pelatantheria insectifera, Phalaenopsis braceana, Pholidota yunnanensis, Schoenorchis scolopendria, S. tixieri, Tainia cordifolia, Thrixspermum calceolum). Пять видов (Dendrobium minusculum, Oberonia simplex, Porpax capuccinorum, P. laosensis и Taeniophyllum filiferum) из Камбоджи и Лаоса описаны в качестве новых для науки. Один род (Didymoplexis) приводится для Лаоса впервые. Такие декоративные виды, как Aerides falcata, A. flabellata, A. rosea, Arundina caespitosa, Ascolabium pusillum, Bulbophyllum bicolor, Calanthe cardioglossa, Coelogyne assamica, C. calcicola, C. eberhardtii, C. flaccida, C. trinervis, Dendrobium aphyllum, D. christyanum, D. chryseum, D. chrysotoxum, D. crepidatum, D. dixanthum, D. falconeri, D. fimbriatum, $D$. findleyanum, D. gratiosissimum, D. jenkinsii, D. loddigesii, D. nobile, D. senile, D. thyrsiflorum, D. trantuanii, D. trigonopus, D. williamsonii, Doritis pulcherrima, Gastrochilus suavis, Holcoglossum kimballianum, H. subulifolium, Hygrochilus parishii, Paphiopedilum concolor, P. dianthum, Phalaenopsis braceana, Schoenorchis scolopendria, S. tixieri, Spathoglottis affinis, Tainia cordifolia, Vanda brunnea, V. denisoniana, V. flavobrunnea в настоящее время на территории изученных локальных флор становятся очень редкими и крайне нуждаются в охране для предотвращения их полного вымирания в природе.
\end{abstract}

\section{Introduction}

The orchid flora of Cambodia and Laos still remains one of the less studied orchid floras in Tropical Asia. In comparison to other countries of Indochina Peninsula, such as Thailand or Vietnam each including at least 1100 species (Seidenfaden, 1992; Averyanov, 2003; Pedersen et al., 2011), about 200 orchid species totally were recorded for Cambodia (Seidenfaden, 1992; Averyanov, 2013; Leti et al., 2013; Schuiteman et al., 2015, 2016; Tagane et al., 2015) and about 530 species for Laos (Newman et al., 2007; Schuiteman et al., 2008; Averyanov, 2013). Meanwhile, according to their square and various natural conditions all countries of Indochina should certainly conceal similar orchid richness and diversity. Present paper summarizes some consequent steps in studies of the orchid flora of Cambodia and Laos based on herbarium specimens collected by different collectors in the field mainly during 2013-2015.

The paper provides 298 new localities for 167 species (from 57 genera) among which 8 species are new for the flora of Cambodia and 43 species represent new records for Laos. One genus - Didymoplexis is recorded for the Laotian flora at first. Mentioned new records (see table 1) represent sig- 
Table 1

New records of orchids in the flora of Cambodia and Laos

Names of new species for science and species new for the flora are marked with bold font (additionally, new species for science are marked with asterisk “*”, new species for Cambodia and Laos are marked with superscript letters "C" and "L" respectively)

\begin{tabular}{|c|c|c|c|}
\hline Acampe rigida & Cleisostoma arietinum & ${ }^{\mathrm{L}}$ D. trantuanii & Odontochilus tortus \\
\hline Aerides falcata & C. birmanicum & D. trigonopus & Panisea tricallosa \\
\hline A. flabellata & C. fuerstenbergianum & ${ }^{\mathrm{L}} D$. williamsonii & P. uniflora \\
\hline A. rosea & ${ }^{\mathrm{L}}$ C. melanorachis & ${ }^{\mathrm{L}}$ Didymoplexis pallens & Paphiopedilum concolor \\
\hline Appendicula hexandra & C. simondii & Doritis pulcherrima & P. dianthum \\
\hline Arachnis labrosa & ${ }^{\mathrm{L}}$ C. subulatum & Epigeneium amplum & Pelatantheria ctenoglossum \\
\hline Arundina caespitosa & ${ }^{\mathrm{L}}$ C. williamsonii & ${ }^{\mathrm{L}}$ E. fuscescens & ${ }^{\mathrm{L}} P$. insectifera \\
\hline Ascolabium pusillum & Coelogyne assamica & Eria acervata & Phaius flavus \\
\hline Biermannia calcarata & C. calcicola & ${ }^{\mathrm{L}}$ E. apertiflora & L Phalaenopsis braceana \\
\hline Bulbophyllum affine & ${ }^{\mathrm{L}}$ C. eberhardtii & ${ }^{\mathrm{L}}$ E. bambusifolia & Pholidota articulate \\
\hline${ }^{\mathrm{L}}$ B. ambrosia & C. flaccida & E. lasiopetala & P. imbricate \\
\hline${ }^{\mathrm{L}} B$. andersonii & ${ }^{\mathrm{L}}$ C. leucantha & E. muscicola & P. pallida \\
\hline B. apodum & ${ }^{\mathrm{L}}$ C. ovalis & ${ }^{\mathrm{L}} \mathrm{E}$. pachyphylla & ${ }^{\mathrm{C}} \boldsymbol{P}$. recurva \\
\hline${ }^{\mathrm{L}}$ B. bicolor & C. trinervis & E. paniculata & ${ }^{\mathrm{L}} \boldsymbol{P}$. yunnanensis \\
\hline${ }^{\mathrm{L}}$ B. bisetoides & C. viscosa & E. pannea & Podochilus oxystophylloides \\
\hline B. clandestinum & Corymborkis veratrifolia & ${ }^{\mathrm{C}}$ E. tenuiflora & Polystachya concreta \\
\hline${ }^{\mathrm{L}} B$. delitescens & Cymbidium aloifolium & E. tomentose & Pomatocalpa spicatum \\
\hline${ }^{\mathrm{L}} B$. depressum & Dendrobium aphyllum & Flickingeria angustifolia & *Porpax capuccinorum \\
\hline B. flabellum-veneris & D. christyanum & ${ }^{\mathrm{L}} \mathrm{F}$. fimbriate & P. elwesii \\
\hline${ }^{\mathrm{L}} \mathrm{B}$. forrestii & D. chrysanthum & ${ }^{\mathrm{L}}$ Gastrochilus suavis & *P. laosensis \\
\hline B. helenae & D. chryseum & Goodyera procera & Robiquetia spathulata \\
\hline${ }^{\mathrm{L}}$ B. lilacinum & D. chrysotoxum & Hetaeria affinis & ${ }^{\mathrm{L}}$ Schoenorchis scolopendria \\
\hline${ }^{\mathrm{L}}$ B. longibrachiatum & D. concinnum & Holcoglossum kimballianum & ${ }^{\mathrm{L}} S$. tixieri \\
\hline${ }^{\mathrm{C}}$ B. macranthum & D. crepidatum & H. subulifolium & Smitinandia micrantha \\
\hline${ }^{\mathrm{C}} B$. orientale & D. dixanthum & Hygrochilus parishii & Spathoglottis affinis \\
\hline B. parviflorum & D. ellipsophyllum & Liparis bootanensis & Sunipia scariosa \\
\hline${ }^{\mathrm{C}}$ B. physocoryphum & ${ }^{\mathrm{L}} D$. eriiflorum & L. cespitosa & *Taeniophyllum filiferum \\
\hline${ }^{\mathrm{L}}$ B. psyhoon & ${ }^{\mathrm{L}} D$. falconeri & ${ }^{\mathrm{L}}$ L. mannii & L Tainia cordifolia \\
\hline B. repens & D. fimbriatum & L. pumila & ${ }^{\mathrm{C}}$ Thelasis pygmaea \\
\hline${ }^{\mathrm{C}}$ B. retusiusculum & D. findleyanum & ${ }^{\mathrm{L}}$ L. sootenzanensis & ${ }^{\mathrm{L}}$ Thrixspermum calceolum \\
\hline B. rufilabrum & D. gratiosissimum & L. viridiflora & T. centipeda \\
\hline B. spathulatum & D. jenkinsii & ${ }^{\mathrm{L}}$ Luisia ramosii & Trias disciflora \\
\hline B. tenuifolium & D. lagarum & L. zollingeri & Trichoglottis lorata \\
\hline B. violaceolabellum & D. loddigesii & Malleola dentifera & T. orchidea \\
\hline B. xylophyllum & D. nobile & Nervilia aragoana & Trichotosia dasyphylla \\
\hline${ }^{\mathrm{L}}$ Calanthe alismifolia & ${ }^{*} D$. minusculum & Oberonia cavaleriei & T. pulvinata \\
\hline C. cardioglossa & ${ }^{\mathrm{C}}$ D. pachyphyllum & O. ensiformis & Tropidia angulosa \\
\hline C. lyroglossa & D. parciflorum & O. gammiei & T. curculigoides \\
\hline C. odora & D. porphyrochilum & ${ }^{\mathrm{L}} O$. kwangsiensis & Vanda brunnea \\
\hline${ }^{\mathrm{L}} C$. sylvatica & D. salaccense & ${ }^{\mathrm{L}}$ O. maxima & $V$. denisoniana \\
\hline Callostylis rigida & D. senile & O. mucronate & V. flavobrunnea \\
\hline${ }^{\mathrm{L}}$ Cheirostylis pusilla & D. thyrsiflorum & *O. simplex & \\
\hline
\end{tabular}

nificant addition to our previous knowledge (Gagnepain, Guillaumin, 1932-1934; Vidal, 1959; Seidenfaden, 1966, 1973, 1975; Schuiteman, Vogel, 2000; Callaghan, 2004; Svengsuksa, Lamxay, 2005) summarized in last accounts of Cambodian (Seidenfaden, 1992) and Laotian floras (Newman et al., 2007; Schuiteman et al., 2008). Five species - Den- drobium minusculum Aver., Oberonia simplex Aver., Porpax capuccinorum Aver., P. laosensis Aver. and Taeniophyllum filiferum Aver. discovered in Cambodia and Laos are described as new for science.

The annotated list of all discovered species is presented below. Paragraphs for species are arranged in the list according to species name in 
alphabetic order. The annotation for each species includes following data in separate lines:

- valid name and main synonyms, occurring in regional literature;

- maximally abbreviated and unified text of herbarium labels including geographical locality, collection date, collectors' names, collection number and acronym of Herbarium where mentioned specimen is housed;

- living form, summarized available data on ecology, habitat elevation, phenology and observed rarity;

- general distribution and distribution in studied countries (by listing of the country provinces).

Color and line illustrations as well as taxonomic notes are additionally provided for new and most significant species. The names of orchid species and genera reported for Cambodia and Laos at first are marked in the list with superscript letters "C" and "L" respectively, and species described as a new for science are marked with asterisk "**”.

The following Herbarium acronyms are used in the text:

CPC - Herbarium of the Center for Plant Conservation, Vietnam Union of Science and Technology Associations, Hanoi, Vietnam.

LE - Herbarium of Komarov Botanical Institute, Russian Academy of Sciences, St. Petersburg, Russian Federation.

HNL - Conseil National des Sciences, National Herbarium of Laos, Vientiane, Lao PDR.

FOF - Herbarium of National University of Laos, Vientiane, Lao PDR.

Prefixes " $C P C$ " and " $L A-V N$ " at some collecting numbers represent abbreviations of "The Center for Plant Conservation" $(C P C)$ and "Laos - Vietnamese expedition" $(L A-V N)$. Both collecting series are attributed to herbaria collected in expeditions managed by the Center for Plant Conservation (Vietnam Union of Science and Technology Associations) with main collectors - Leonid V. Averyanov and Nguyen Tien Hiep. Prefixes " $K$ " and " $T M$ " at plant collecting numbers designate herbarium collections of Eugene L. Konstantinov and Tatiana V. Maisak with their co-workers.

Attractive species highly desirable for cultivation are indicated in the list as "ornamental". Almost all these species fast become very rare due to commercial collecting even on protected areas and badly need conservation to prevent their full extinction in nature.

\section{New and rare orchids in the flora of Cambodia and Laos}

Acampe Lindl.

A. rigida (J. E. Smith) P. F. Hunt,

1970, Kew Bull. 24, 1: 98; Newman et al., 2007, Checklist Vasc. P1. Lao PDR: 251; Schuit. et al., 2008, Nord. Journ. Bot. 26: 262; Aver., 2013, Turczaninowia 16, 4: 28.

Northeastern Laos, Houaphan province, Viengxay district, Vieng Xai village, 7 April 2015. N. T. Hiep, L. Averyanov, N. S. Khang et al., LAVN 1144 (HNL, FOF, LE). Northeastern Laos, Houaphan province, Viengxay district, Xieng Luong village, 8 April 2015, N. T. Hiep, L. Averyanov, N. S. Khang et al., LA-VN 1163 (HNL, FOF, LE).

Epiphyte and lithophyte. Primary and secondary broad-leaved evergreen forests on limestone, often on exposed cliffs near hilltops, 200-950 m. Fl. September - November. One of the commonest species in observed limestone areas.

Southern and east Africa, Sri Lanka, India, Myanmar, China, Thailand, Cambodia, Vietnam, Peninsular Malaysia. Laos: Attapu, Bolikhamxai, Champasak, Houaphan, Khammouan, Louangphrabang, Phongsali, Savannakhet, Vientiane, Xaignabouri.

\section{Aerides Lour.}

A. falcata Lindl. et Paxton,

1851, Fl. Gard. 2: 142; Newman et al., 2007, Checklist Vasc. P1. Lao PDR: 252; Schuit. et al., 2008, Nord. Journ. Bot. 26: 263.

Northeastern Laos, Houaphan province, Hem district, Nacoc village, 12 April 2015, N. T. Hiep, $L$. Averyanov, N. S. Khang et al., LA-VN 1433 (HNL, FOF, LE).

Epiphyte. Primary and secondary broad-leaved evergreen open forests on any kind of soil, 170-1200 m. Fl. March - June. Locally common. Ornamental.

NE. India, Myanmar, Thailand, Cambodia, Vietnam. Laos: Bolikhamxai, Champasak, Houaphan, Khammouan, Louangphrabang, Xaisomboun.

\section{A. flabellata Downie,}

1925, Bull. Misc. Inform. Kew 1925(9): 387;

Newman et al., 2007, Checklist Vasc. Pl. Lao PDR: 252; Schuit. et al., 2008, Nord. Journ. Bot. 26: 314.

$\equiv$ Vanda flabellata (Downie) Christenson, 1985, Indian Orchid Journ. 1, 4: 156. 
Northeastern Laos, Houaphan province, Hem district, Nacoc village, 12 April 2015, N. T. Hiep, $L$. Averyanov, N. S. Khang et al., LA-VN 1419 (HNL, FOF, LE).

Epiphyte. Primary and secondary broad-leaved evergreen and deciduous open dry forests on any kind of soil, often in dipterocarp forests or forests on limestone, (500)1000-1200 m. Fl. April - May. Not common. Ornamental.

SW. China, Thailand, Vietnam. Laos: Bokeo, Bolikhamxai, Houaphan, Louang Namtha, Louangphrabang.

\section{A. rosea Paxton,}

1851, Fl. Gard. 2: 109; Schuit. et al., 2008, Nord. Journ. Bot. 26: 263; Aver., 2013, Turczaninowia 16, 4: 28.

Northeastern Laos, Houaphan province, Xon district, Nong Hua Pa Noi village, 11 April 2015, N. T. Hiep, L. Averyanov, N. S. Khang et al., LA-VN 1235 (HNL, FOF, LE). Northeastern Laos, Houaphan province, Hem district, Na Puok village, 11 April 2015, N. T. Hiep, L. Averyanov, N. S. Khang et al., LA-VN 1322 (HNL, FOF, LE).

Epiphyte and occasionally lithophyte. Primary and secondary broad-leaved evergreen forests on rocky limestone, 500-1200 m. Fl. March - May. Locally common. Ornamental.

NE. India, Bhutan, Myanmar, S. China, Thailand, Vietnam. Laos: Houaphan, Louangphrabang, Vientiane.

\section{Appendicula Blume}

A. hexandra (J. Koenig) J. J. Sm.,

1932, Bull. Jard. Bot. Buitenzorg 3, 12: 119; Aver., 2013, Turczaninowia 16, 4: 28.

Southern Cambodia, Kampot province, Phnom Bokor National park, 1 December 2014, E. Konstantinov, $K$-365 (LE).

Epiphyte and occasionally lithophyte. Cloud evergreen forests on mountain tops, usually along rocky streams. 900-100 m. Fl. May - July. Occasional.

Thailand, Vietnam, Sumatra. Cambodia: Kampot.

Arachnis Blume

A. labrosa (Lindl. et Paxton) Rchb. f.,

1886, Bot. Centralbl. 28: 343; Newman et al., 2007, Checklist Vasc. Pl. Lao PDR: 253; Schuit. et al., 2008, Nord. Journ. Bot. 26: 265; Aver., 2013, Turczaninowia 16, 4: 28.

$=$ Renanthera bilinguis Rchb. f., 1854, Xenia Orchid. 1: 7. $\equiv$ Renanthera labrosa (Lindl. et Paxton) Rchb. f., 1855, Xenia Orchid. 1: 88.

$\equiv$ Armodorum labrosum (Lindl. et Paxton) Schltr., 1911, Repert. Spec. Nov. Regni Veg. 10: 197.

Northeastern Laos, Houphan province, Hem district, Nacoc village, 12 April 2015, N. T. Hiep, L. Averyanov, N. S. Khang et al., LA-VN 1463 (HNL, FOF, LE). Northeastern Laos, Houphan province, Viengxay district, Vieng Xai village, 7 April 2015, N. T. Hiep, L. Averyanov, N. S. Khang et al., LA-VN 1107 (HNL, FOF, LE).

Epiphyte. Primary and secondary broad-leaved evergreen and deciduous open dry forests on any kind of soil, often in dipterocarp forests or on forests on limestone, 150-1500 m. Fl. July - August. Locally common.

NE. India, Bhutan, Myanmar, S. China, Ryukyu Is., Thailand, Vietnam. Laos: Attapu, Champasak, Houaphan, Saravan, Vientiane.

\section{Arundina Blume}

A. caespitosa Aver.,

2007, Taiwania 52: 289; Schuit. et al., 2008, Nord. Journ. Bot. 26: 265.

Fig. 1A.

Central Laos, Vientiane province, Phou Khao Khouay National Protected Area to about $40 \mathrm{~km} \mathrm{NE}$ of Vientiane, 9 December 2015, L. Averyanov, T. Maisak, AL 159a (LE - photo).

Lithophyte. Primary and secondary dry evergreen forests along rocky stream, commonly of wet sandstone rocks, rarely on sandy wet soil in deciduous dipterocarp forests, 300-800 m. Fl. August - December. Locally common. Ornamental.

Vietnam. Laos: Bolikhamxai, Khammouan, Vientiane.

This rare species almost extinct in Vietnam but remains still common fairly in some observed areas in central Laos (Phou Khao Khouay National Protected Area).

\section{Ascolabium S. S. Ying}

A. pusillum (Aver.) Aver.,

1994, Identification Guide. Viet. Orch.: 397; Schuit. et al., 2008, Nord. Journ. Bot. 26: 265.

$\equiv$ Ascocentropsis pusilla (Aver.) Senghas et Schildh., 2000, Orchideenfr. 7: 290; Newman et al., 2007, Checklist Vasc. Pl. Lao PDR: 253.

三Ascocentrum pusillum Aver., 1988, Bot. Journ. (Moscow \& Leningrad) 73: 104.

Southwestern Laos, Champasak province, Pakse district, Bolaven plateau, along road Paksong - Ban Houay Kong, 26 November 2015, E. Konstantinov, T. Vongsa, K-389 (LE). 


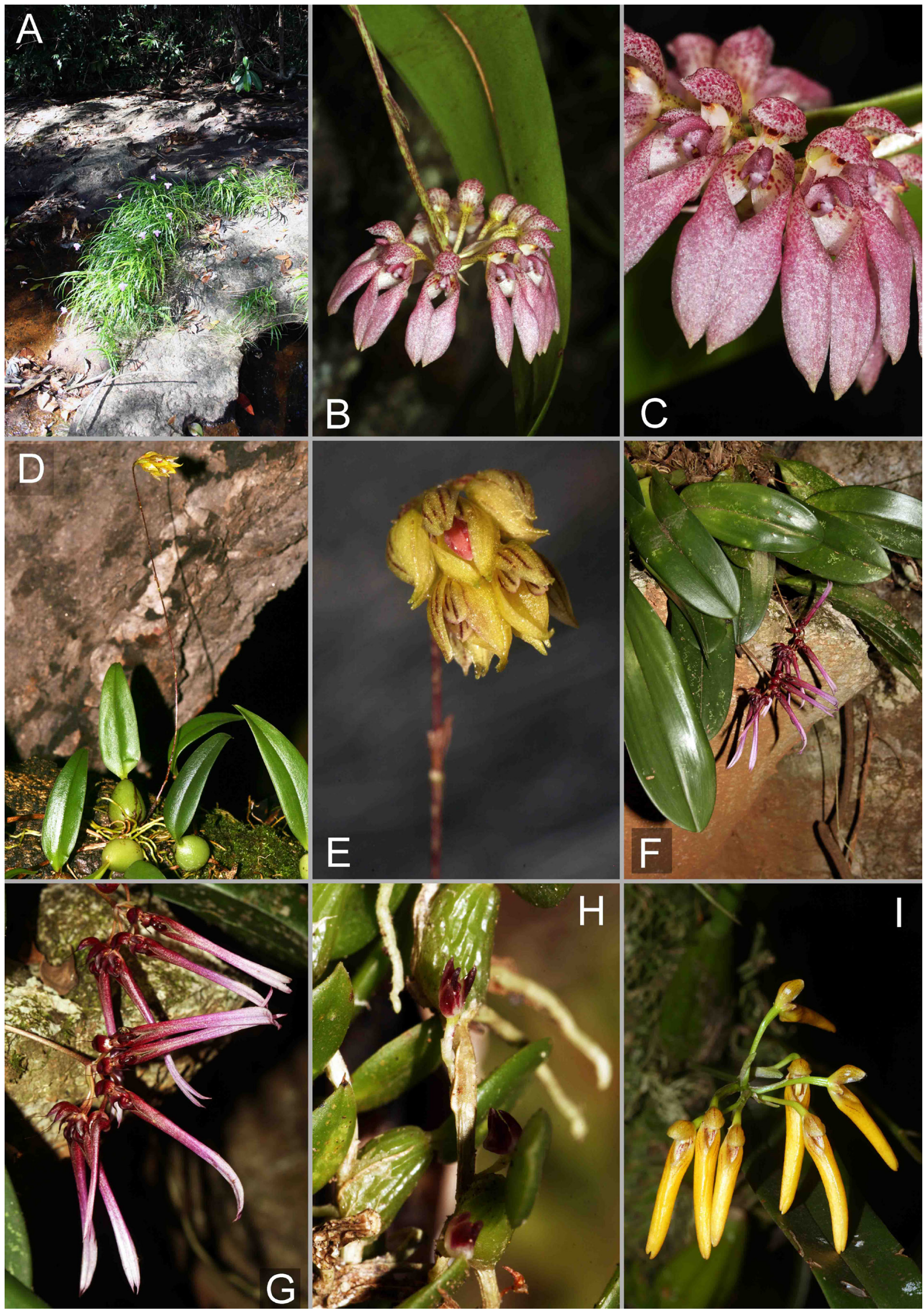

Fig. 1. New and rare orchids in the flora of Cambodia and Laos. A - Arundina caespitosa Aver. (AL 159a). B, C B. andersonii (Hook. f.) J. J. Sm. (LA-VN 1184a). D, E - Bulbophyllum bisetoides Seidenf. (LA-VN 514). F, G B. delitescens Hance (LA-VN 954). H - B. depressum King et Pantl. (LA-VN 1157). I - B. forrestii Seidenf. (LA-VN 516). Photos by L. Averyanov. 
Epiphyte. Primary and old secondary evergreen and deciduous open forests, $800-1200 \mathrm{~m}$. Fl. November - December. Rare. Ornamental.

Thailand, Vietnam. Laos: Bolikhamxai, Champasak.

Rare endemic of eastern Indochina standing in Vietnam on the verge of full extinction.

Biermannia King et Pantl.

B. calcarata Aver.,

1988, Bot. Journ. (Moscow \& Leningrad) 73: 429; Schuit. et al., 2008, Nord. Journ. Bot. 26: 265.

Central Laos, Xiangkhouang province, Peak district, Khoang village, 3 April 2015, N. T. Hiep, L. Averyanov, N. S. Khang et al., LA-VN 972 (HNL, FOF, LE).

Canopy epiphyte. Primary and secondary broadleaved evergreen humid forests on any kind of soil, commonly along stream valleys, 900-1450 m. Fl. May - June. Rare.

Vietnam. Laos: Phongsali, Xiangkhouang.

Plants from Laos may be different from the type originated from northern Vietnam (Schuiteman et al., 2008).

\section{Bulbophyllum Thouars}

B. affine Lindl.,

1830, Gen. Sp. Orch. Pl.: 48; Newman et al., 2007, Checklist Vasc. P1. Lao PDR: 254; Schuit. et al., 2008, Nord. Journ. Bot. 26: 266; Aver., 2013, Turczaninowia 16, 4: 29.

Northeastern Laos, Houaphan province, Viengxay district, Xieng Luong village, 8 April 2015, N. T. Hiep, L. Averyanov, N. S. Khang et al., LA-VN 1184 (HNL, FOF, LE).

Primary and secondary broad-leaved evergreen and deciduous dry forests on any kind of soil, 600 1500 m. Fl. May - August.

NE. India, Nepal, Bhutan, China, Ryukyu Islands, Thailand, Vietnam, Cambodia. Laos: Bolikhamxai, Houaphan, Khammouan, Louang Namtha, Phongsali, Saravan, Vientiane.

It is "one of the most frequent epiphytic orchids in northern Laos" (Schuiteman et al., 2008).

${ }^{\mathrm{L}}$ B. ambrosia Schltr.,

1919, Repert. Spec. Nov. Regni Veg. Beih. 4: 247.

= B. amygdalinum Aver., 1988, Bot. Journ. (Moscow \& Leningrad) 73: 1023.

Northeastern Laos, Houaphan province, Viengxay district, Vieng Xai village, 7 April 2015, N. T. Hiep, L. Averyanov, N. S. Khang et al., LA-VN 1152 (HNL, FOF, LE). Northeastern Laos, Houaphan province, Xon district, Nong Hua Pa Noi village, 11 April 2015, N. T. Hiep, L. Averyanov, N. S. Khang et al., $L A-V N 1240$ (HNL, FOF, LE).

Creeping epiphyte. Primary and secondary broad-leaved evergreen dry forests, often on rocky limestone, 800-1200 m. Fl. December - April. Locally very common.

S. China, Hong Kong, Hainan, Vietnam. Laos: Houaphan.

${ }^{\mathrm{L}}$ B. andersonii (Hook. f.) J. J. Sm.,

1912, Bull. Jard. Bot. (Buitenzorg) 2 ser. 8: 22.

Fig. 1B, C.

Northeastern Laos, Houaphan province, Viengxay district, Xieng Luong village, 8 April 2015, N. T. Hiep, L. Averyanov, N. S. Khang et al., LA-VN $1184 a$ (LE).

Primary and secondary broad-leaved evergreen dry forests on rocky limestone, 600-700 m. Fl. MayAugust. Not common.

NE. India, Bhutan, China, Myanmar. Laos: Houaphan.

B. apodum Hook. f.,

1890, Fl. Brit. India 5: 766; Aver., 2013, Turczaninowia 16, 4: 29.

Creeping lithophyte and epiphyte. Northeastern Laos, Houaphan province, Hem district, Nacoc village, 12 April 2015, N. T. Hiep, L. Averyanov, N. S. Khang et al., LA-VN 1441 (HNL, FOF, LE).

Creeping epiphyte and lithophyte. Primary and secondary broad-leaved evergreen open dry forests on rocky limestone, 500-1200 m. Fl. May - June. Locally very common.

NE. India, Thailand, Vietnam, Malaysia, Sumatra, Java, Kalimantan. Laos: Houaphan, Vientiane.

${ }^{\mathrm{L}}$ B. bicolor Lindl.,

1830, Gen. Sp. Orch. Pl.: 49; Aver., 2012, Taiwania, 57, 2: 127.

Northeastern Laos, Houphan province, Viengxay district, Vieng Xai village, 7 April 2015, N. T. Hiep, L. Averyanov, N. S. Khang et al., LA-VN 1152a / TM 1271 (LE).

Fig. 2. (d-EXSICCATES OF VIETNAMESE FLORA 0252/LA-VN 1152a).

Creeping epiphyte and occasionally lithophyte. Primary and secondary broad-leaved evergreen open forests on rocky limestone, commonly on mossy hill and mountain tops, $800-950 \mathrm{~m}$. Fl. MayJune. Locally common. Ornamental.

SE. China (Hong Kong), NW. Vietnam. Laos: Houphan. 
This species considered earlier as endemic of Hong Kong (Barretto, Cribb, Gale, 2011) has actually broad distribution in limestone areas of northeastern Laos and northwestern Vietnam. It is noteworthy that in Indochina the species was observed exclusively in forests on rocky limestone.

${ }^{\mathrm{L}}$ B. bisetoides Seidenf.,

1970, Bot. Tidsskr. 65: 354.

Fig. 1D, E.

Central Laos, Vientiane province, Vang Vieng district, Na Po village, about $12 \mathrm{~km}$ to the $\mathrm{W}$ of Vang Vieng town, 17 March 2013, N. T. Hiep, L. Averyanov et al., LA-VN 514 (LE).

Epiphyte. Primary and secondary dry broadleaved evergreen and semideciduous forests on rocky limestone, 500-1000 m. Fl. December. Rare.

Thailand, Vietnam. Laos: Vientiane.

\section{B. clandestinum Lindl.,}

1841, Bot. Reg. 27, misc.: 77; Newman et al., 2007, Checklist Vasc. Pl. Lao PDR: 955; Schuit. et al., 2008, Nord. Journ. Bot. 26: 267.

= B. sessile (Kuntze) J. J. Sm., 1905, Orch. Java: 448.

$=$ B. bolovenense Guillaumin, 1958, Bull. Mus. Natl. Hist. Nat. 2I, 29: 500.

Central Laos, Xiangkhouang province, Kham district, Huad village, 4 April 2015, N. T. Hiep, L. Averyanov, N. S. Khang et al., LA-VN 1088 (HNL, FOF, LE).

Lithophyte and epiphyte. Primary and secondary broad-leaved evergreen and deciduous dry forests on any kind of soil, often along streams, 170-1400 m. Fl. June - August. Occasional.

Thailand, Vietnam, Malacca Peninsula, Singapore, Sumatra, Java, Borneo, Sulawesi, Moluccas, New Guinea, Philippines, tropical Pacific islands. Laos: Bolikhamxai, Champasak, Khammouan, Vientiane, Xiangkhouang.

\section{${ }^{\mathrm{L}}$ B. delitescens Hance,}

1876, Journ. Bot. 14: 44.

Fig.1F, G.

Central Laos, Xiangkhouang province, Kham district, Huad village, 4 April 2015, N. T. Hiep, L. Averyanov, N. S. Khang et al., LA-VN 1033 (HNL, FOF, LE). Central Laos, Xiangkhouang province, Peak district, Khoang village, 3 April 2015, N. T. Hiep, L. Averyanov, N. S. Khang et al., LA-VN 954 (HNL, FOF, LE). Northeastern Laos, Houaphan province, Viengxay district, Xieng Luong village, 8 April 2015, N. T. Hiep, L. Averyanov, N. S. Khang et al., $L A-V N 1180$ (HNL, FOF, LE).

Creeping epiphyte and lithophyte. Primary and secondary broad-leaved evergreen and semideciduous dry forests on rocky limestone, commonly on shady cliffs, $600-1450 \mathrm{~m}$. Fl. March May. Locally common. Ornamental.

NE. India, continental SE. China, Hong Kong, Hainan, Vietnam. Laos: Houaphan, Xiangkhouang.

${ }^{\mathrm{L}}$ B. depressum King et Pantl.,

1897, Journ. Asiat. Soc. Bengal, 2, Nat. Hist. 66: 585.

= B. hastatum Tang et F. T. Wang, 1974, Acta Phytotax. Sin. 12: 44.

Fig. $1 \mathrm{H}$.

Northeastern Laos, Houaphan province, Viengxay district, Xieng Luong village, 8 April 2015, $N$. T. Hiep, L. Averyanov, N. S. Khang et al., LA-VN 1157 (HNL, FOF, LE).

Epiphyte. Primary and secondary broad-leaved evergreen dry forests on rocky limestone, commonly on hill and mountain tops, 600-700 m. Fl. April May. Not common.

N. India, S. China, Myanmar, Thailand, Laos, Vietnam, Java, Sumatra, Kalimantan. Laos: Houaphan.

B. flabellum-veneris (J. Koenig) Aver. 2003, Updated Checklist Orchids Vietnam: 73.

= B. lepidum (Blume) J. J. Sm., 1905, Orch. Java: 471; Seidenf., 1992, Opera Bot. 114: 279.

Southern Cambodia, to the N of Sihanoukville town, Koch Rong Island in Siam Gulf, 20 November 2011, T. Maisak, L. Osinovetz, M. Telepova, 729 (LE).

Epiphyte. Secondary dry evergreen broad-leaved lowland forest, 50-600 m. Fl. April - October. Not rare. Ornamental.

Myanmar, Andaman Islands, Thailand, Laos, Vietnam, Malacca Peninsula, Singapore, Sumatra, Java, Borneo, Sulawesi, tropical Pacific islands. Cambodia: Siem Reap, Sihanoukville.

${ }^{\mathrm{L}}$ B. forrestii Seidenf., 1974, Dansk Bot. Ark. 29, 1: 120, fig. 60.

Fig. 1I; 3A.

Central Laos, Vientiane province, Vang Vieng district, Na Po village, about $12 \mathrm{~km}$ to the $\mathrm{W}$ of Vang Vieng town, 17 March 2013, N. T. Hiep, L. Averyanov, K. Chantthavongsa et al., LA-VN 516 (HNL, FOF, LE).

Epiphyte or occasionally lithophyte. Primary and secondary dry broad-leaved evergreen and 


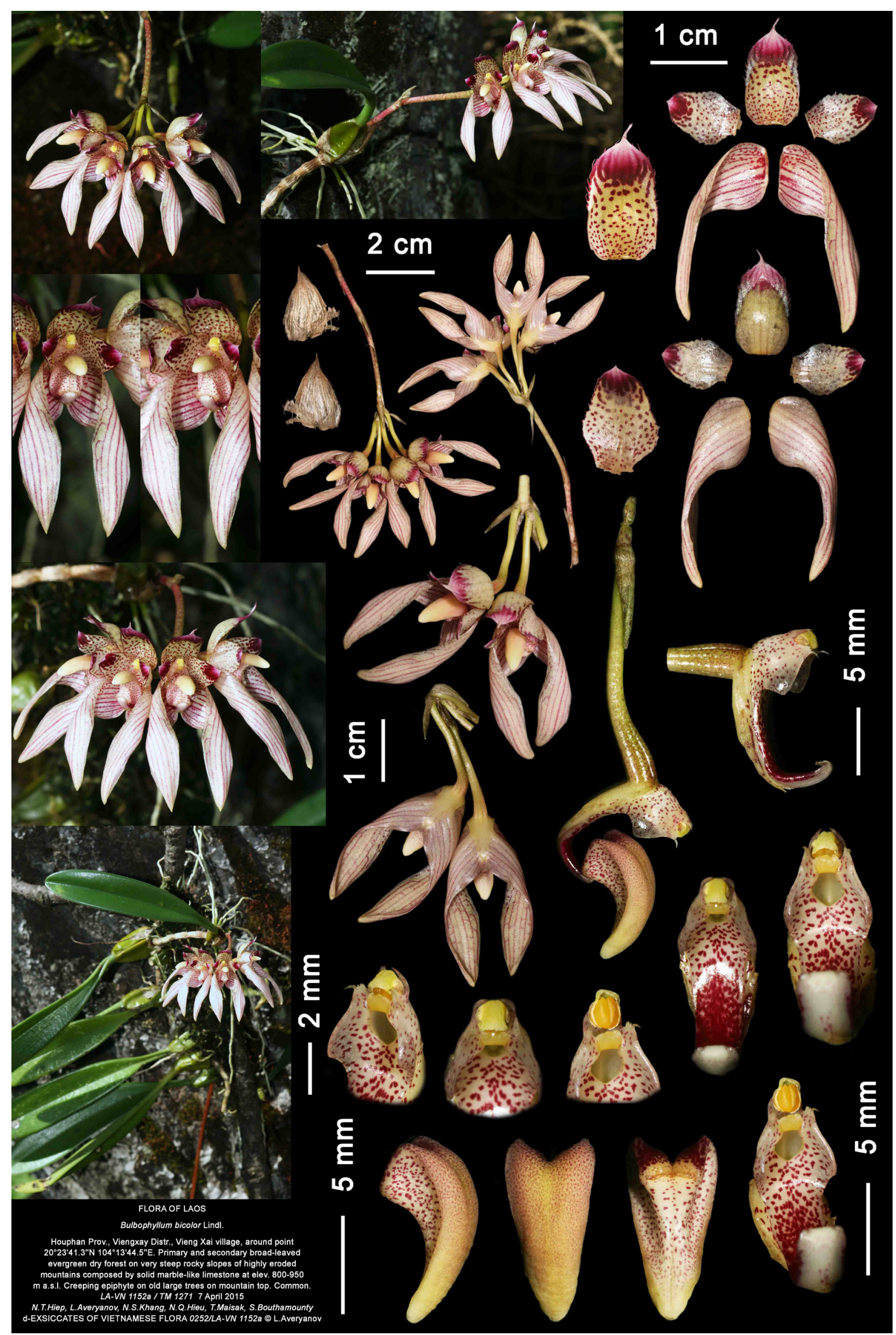

Fig. 2. New and rare orchids in the flora of Cambodia and Laos. Bulbophyllum bicolor Lindl. Digital herbarium specimen - d-EXSICCATES OF LAOTIAN FLORA 0252/LA-VN 1152a / TM 1271. Photos, design and image correction by L. Averyanov and T. Maisak. 

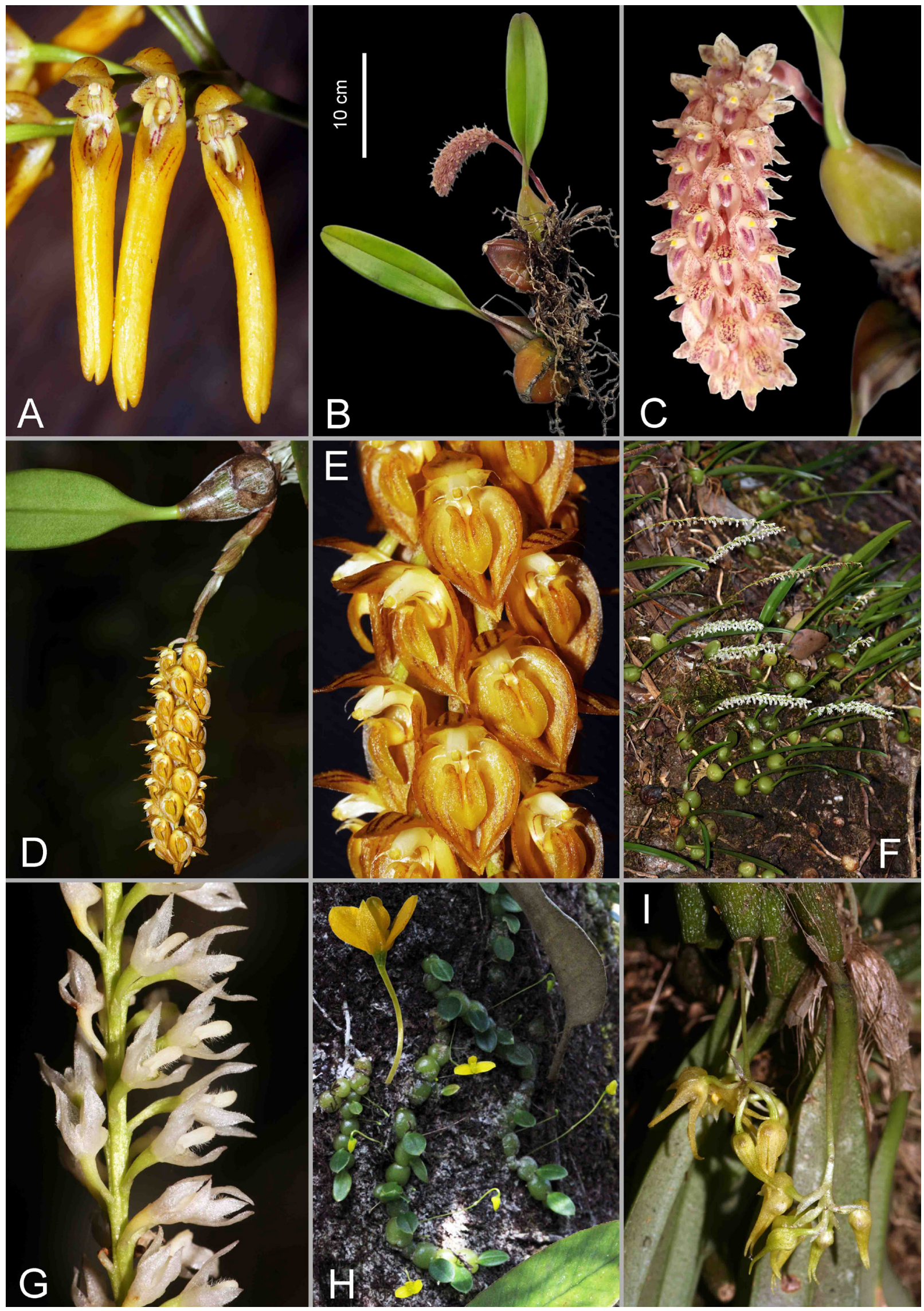

Fig. 3. New and rare orchids in the flora of Cambodia and Laos. A - Bulbophyllum forrestii Seidenf. (LA-VN 516). B, $\mathrm{C}-$ B. lilacinum Ridl. (K-387). D, E - B. orientale Seidenf. (LA-VN 1241a / TM 1287). F, G - B. parviflorum Par. et Rchb. f. (AL 154). H - B. physocoryphum Seidenf. (K-366). I - B. psyhoon Schltr. (LA-VN 613). Photos by L. Averyanov (A, D-G, I) and E. Konstantinov (D, C, H). 
semideciduous forests on rocky limestone, commonly on hill tops, 500-1000 m. Fl. DecemberFebruary. Not common.

Myanmar, SW. China (Yunnan), Thailand, Vietnam. Laos: Vientiane.

Flowers with very strong unpleasant smell.

B. helenae J. J. Sm.,

1912, Bull. Jard. Bot. Buitenzorg 2, 8: 24; Newman et al., 2007, Checklist Vasc. Pl. Lao PDR: 255; Schuit. et al., 2008, Nord. Journ. Bot. 26: 267.

Northeastern Laos, Houaphan province, Xon district, Nong Hua Pa Noi village, 11 April 2015, N. T. Hiep, L. Averyanov, N. S. Khang et al., LA-VN 1241 (HNL, FOF, LE).

Creeping epiphyte. Primary and secondary broad-leaved evergreen forests on any kind of soil, 900-1250 m. Fl. March - May, August - September. Not common.

NE. India, Nepal, Myanmar, Thailand. Laos: Houaphan, Louang Namtha, Phongsali.

${ }^{\mathrm{L}}$ B. lilacinum Ridl., 1897, Journ. Linn. Soc., Bot. 32: 276.

Fig. 3B, C.

Southwestern Laos, Champasak province, Pakse district, Bolaven plateau, road Paksong - Ban Houay Kong, 1170 m, 21 November 2015, E. Konstantinov, T. Vongsa, K-387 (LE).

Creeping epiphyte. Primary and secondary evergreen and deciduous broad-leaved open dry forests, 1100-1200 m. Fl. November. Rare.

Thailand, Malacca Peninsula. Laos: Champasak.

${ }^{\mathrm{L}}$ B. longibrachiatum Z. H. Tsi, 1981, Bull. Bot. Res., Harbin 1: 115.

= B. purpureifolium Aver., 1997, Bot. Journ. (Moscow \& Leningrad) 82: 141.

Central Laos, Xiangkhouang province, Peak district, Khoang village, 3 April 2015, N. T. Hiep, L. Averyanov, N. S. Khang et al., LA-VN 988 (HNL, FOF, LE).

Creeping lithophyte or occasionally epiphyte. Primary and secondary broad-leaved evergreen dry forests on rocky limestone, commonly on hill and mountain tops, 1200-1450 m. Fl. September October. Locally common. Ornamental.

SE. China (SE. Yunnan), N. Vietnam. Laos: Xiangkhouang.

${ }^{\mathrm{C}}$ B. macranthum Lindl.,

1844, Bot. Reg. 30, tab. 13.

Southern Cambodia, to the $\mathrm{N}$ of Sihanoukville town, Koch Rong Island in Siam Gulf, 20 November
2011, T. Maisak, L. Osinovetz, M. Telepova, 843 (LE).

Creeping epiphyte. Primary and secondary dry evergreen broad-leaved lowland forests on low hills, 20-150 m. Fl. March - May. Locally common.

Myanmar, Thailand, Vietnam, Malacca Peninsula, Sumatra, Java, Kalimantan, Bangka, Philippines, New Guinea, tropical Pacific islands. Cambodia: Sihanoukville.

${ }^{\mathrm{C}}$ B. orientale Seidenf.,

1979, Dansk Bot. Ark. 33: 138; Aver. et Averyanova, 2003, Updated Checklist Orch. Viet.: 16; Newman et al., 2007, Checklist Vasc. Pl. Lao PDR: 256; Schuit. et al., 2008, Nord. Journ. Bot. 26: 270.

Fig. 3D, E.

Southern Cambodia, about 16 kilometers to the $\mathrm{N}$ of Sihanoukville town, Khan Prey Nup district, Kbal Chhay Waterfall, 5 December 2012, T. Maisak, L. Osinovetz, M. Telepova, 971 (LE). Northeastern Laos, Houaphan province, Xon district, Nong Hua Pa Noi village, 11 April 2015, N. T. Hiep, L. Averyanov, N. S. Khang et al., LA-VN 124la / TM 1287 (LE).

Creeping epiphyte and lithophyte. Primary and secondary broad-leaved evergreen and semideciduous forests on any kind of soil, (150)6001200 m. Fl. October - November. Occasional.

Thailand, Vietnam. Cambodia: Sihanoukville. Laos: Bolikhamxai, Houaphan.

B. parviflorum Par. et Rchb. f.,

1874, Trans. Linn. Soc. London 30: 152; Schuit. et al., 2008, Nord. Journ. Bot. 26: 270; Aver., 2013, Turczaninowia 16, 4: 30.

Fig. 3F, G.

Central Laos, Vientiane province, Phou Khao Khouay National Protected Area to about $40 \mathrm{~km} \mathrm{NE}$ of Vientiane, 9 December 2015, L. Averyanov, T. Maisak, AL 154 (LE - photo).

Creeping epiphyte and lithophyte. Primary and secondary dry evergreen forests on any kind of soil, commonly on large rocks along streams, 300-350 m. Fl. November - December. Not rare.

NE. India, Bhutan, Burma, Thailand, Vietnam, Cambodia. Laos: Bolikhamxai, Vientiane.

${ }^{\mathrm{C}}$ B. physocoryphum Seidenf., 1979, Dansk Bot. Ark. 33: 51.

Fig. $3 \mathrm{H}$.

Southern Cambodia, Kampot province, Phnom Bokor National park, 16 December 2014, E. Konstantinov, $K-366$ (LE). 
Epiphyte. Cloud evergreen broad-leaved forests near mountain tops, commonly along stream, 9001000 m. Fl. December - January. Rare.

Thailand. Cambodia: Kampot.

${ }^{\mathrm{L}}$ B. psyhoon Schltr.,

1878, Gard. Chron. n.s., 1878: 170.

=B. lockii Aver. et Averyanova, 2006, Komarovia 4: 5.

Fig. 3I; 4A.

Central Laos, Khammouan province, Bounlapha district, Thong Sam village, Hinnam No Protected Area, 9 March 2013, L. Averyanov, N. T. Hiep, $V$. Lamxay et al., LA-VN 319 (HNL, FOF, LE). Central Laos, Vientiane province, Kasi district, Thong Mout village, 20 March 2013, N. T. Hiep, L. Averyanov, $N$. S. Khang et al., LA-VN 613 (HNL, FOF, LE).

Epiphyte. Primary evergreen broad-leaved and mixed forests on any kind of soil, commonly on mountain tops, 800-1650 m. Fl. March - April. Locally very common.

NE. India, S. China, Vietnam. Laos: Khammouan, Vientiane.

\section{B. repens Griff.,}

1851, Not. Pl. Asiat. 3: 293; Schuit. et al., 2008, Nord. Journ. Bot. 26: 270.

Southwestern Laos, Champasak province, Pakse district, Bolaven plateau, road Paksong - Ban Houay Kong, 1170 m, 21 November 2015, E. Konstantinov, T. Vongsa, K-386 (LE).

Creeping lithophyte and epiphyte. Primary and secondary broad-leaved open evergreen and semideciduous forests, mostly on rocky limestone, 1100 1200 m. Fl. November - January. Not common.

NE. India, Myanmar, Thailand, Vietnam. Laos: Champasak.

${ }^{\mathrm{C}} B$. retusiusculum Rchb.f.,

1869, Gard. Chron. 1869: 1182; Newman et al., 2007, Checklist Vasc. Pl. Lao PDR: 256; Schuit. et al., 2008, Nord. Journ. Bot. 26: 270.

Southern Cambodia, Kampot province, Phnom Bokor National park, 16 December 2014, E. Konstantinov, K-367 (LE). Southeastern Laos, Secong province, Thateng district, Bolaven Plateau, 16 December 2015, E. Konstantinov, T. Vongsa, $K-401$ (LE).

Epiphyte. Primary and secondary evergreen broad-leaved forests on any kind of soil, commonly on mountain tops, 900-1700 m. Fl. December April. Not rare.

NE. India, Nepal, Bhutan, Myanmar, Taiwan, Hainan, Thailand, Vietnam, Malacca Peninsula.
Cambodia: Kampot. Laos: Champasak, Secong, Xiangkhouang.

B. rufilabrum Hook. f.,

1890, Fl. Brit. India 5: 761; Newman et al., 2007, Checklist Vasc. Pl. Lao PDR: 256; Schuit. et al., 2008, Nord. Journ. Bot. 26: 271; Aver., 2013, Turczaninowia 16, 4: 30.

Central Laos, Vientiane province, Phou Khao Khouay National Protected Area to about $40 \mathrm{~km}$ NE of Vientiane, 9 December 2015, L. Averyanov, T. Maisak, AL 158 (LE). Central Laos, Vientiane province, Vang Vieng district, Nathong village, about $5 \mathrm{~km}$ to the W of Vang Vieng town, 14 March 2013, N. T. Hiep, L. Averyanov, N. S. Khang et al., $L A-V N 385 b$ (LE).

Creeping epiphyte and lithophyte. Primary and secondary dry evergreen broad-leaved forests on any kind of soil, commonly along streams, 300-500 m. Fl. November - December. Locally very common.

Myanmar, Thailand, Cambodia. Laos: Bolikhamxai, Khammouan, Vientiane.

B. spathulatum (E.W. Cooper) Seidenf.,

1970, Bot. Tidsskr. 65: 347; Newman et al., 2007, Checklist Vasc. P1. Lao PDR: 256; Schuit. et al., 2008, Nord. Journ. Bot. 26: 271.

Fig. 4B, C.

Central Laos, Bolikhamxai province, Khamkeut district, Dan Khum Ngan locality, 18 January 2009, S. Bounphanmy, N. T. Hiep, L. Averyanov et al., $L A-V N 21 j$ (LE). Central Laos, Vientiane province, Phou Khao Khouay National Protected Area to about $40 \mathrm{~km}$ NE of Vientiane, 9 December 2015, $L$. Averyanov, T. Maisak, AL 154a (LE - photo).

Creeping epiphyte. Primary and secondary broadleaved evergreen and semideciduous open forests on thin soil, commonly along rivers or granite outcrops, 300-600 m. Fl. October - December. Occasional.

NE. India, Myanmar, Thailand, Vietnam. Laos: Bolikhamxai, Khammouan, Vientiane.

B. tenuifolium (Blume) Lindl., 1830, Gen. Sp. Orch. P1.: 50.

$=$ B. konstantinovii Aver., 2013, Turczaninowia 16, 4: 29 .

Southern Cambodia, Kampot province, Bokor National Park, Bokor mt., 24 November 2012, T. Maisak, M. Telepova, L. Osinovetz, I. Kutuzova s.n. (LE). Southern Cambodia, Kampot province, Bokor National park, 16 December 2014, E. Konstantinov, $K-368$ (LE).

Epiphyte. Cloud evergreen broad-leaved and mixed forests on mountain tops, often along rocky 


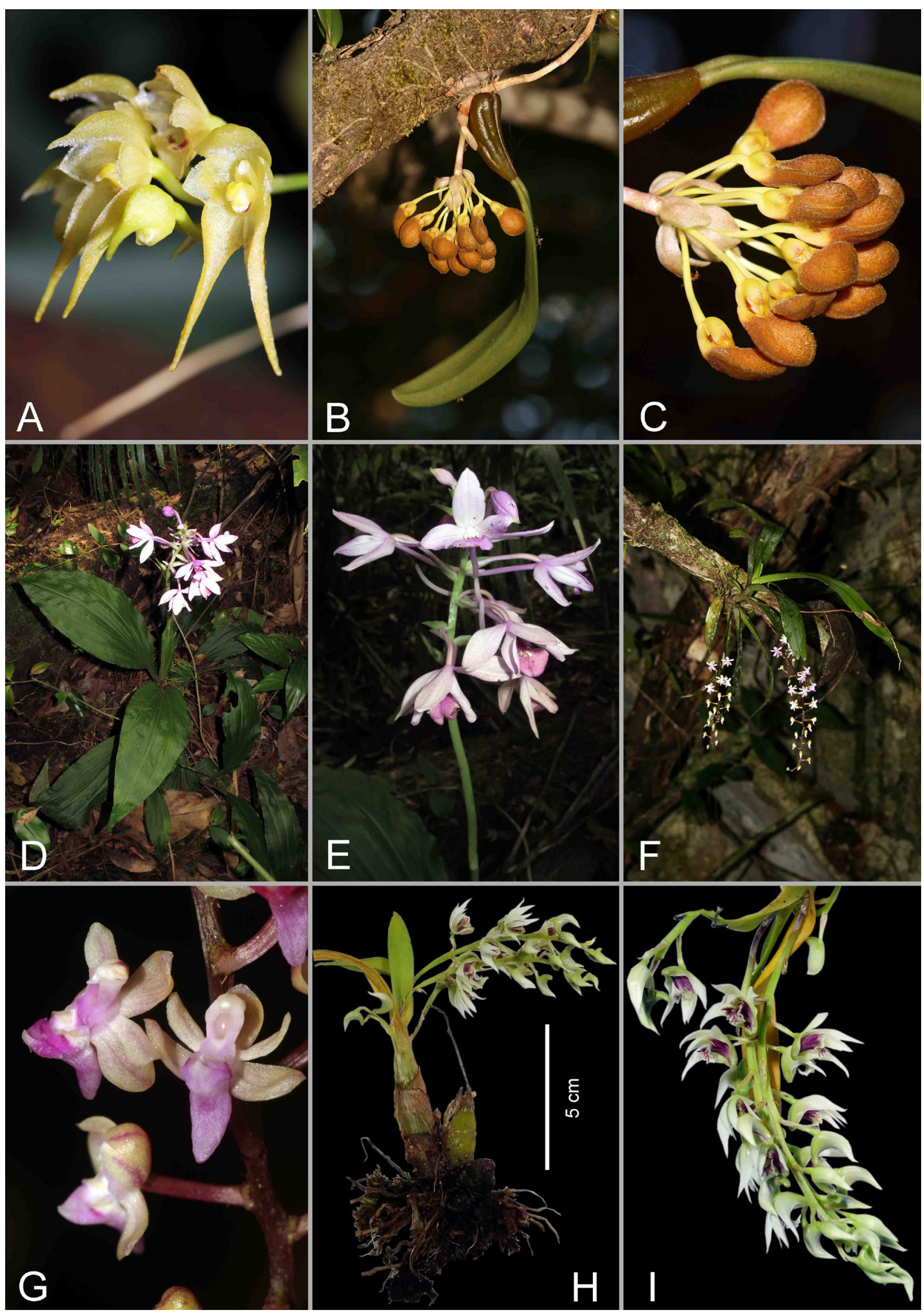

Fig. 4. New and rare orchids in the flora of Cambodia and Laos. A - B. psyhoon Schltr. (LA-VN 319). B, C - B. spathulatum (E. W. Cooper) Seidenf. (AL 154a). D, E - C. sylvatica (Thouars) Lindl. (P. Thomas, R. Timmins s.n.). F, G C. melanorachis Aver. et Averyanova (LA-VN 1176). H, I - D. eriiflorum Griff. (K-391). Photos by L. Averyanov (A-C, F, G), P. Thomas, R. Timmins (D, E) and E. Konstantinov (H, I). 
mountain streams, 900-1500 m. Fl. November December. Not common.

Thailand, Malaysia, Sumatra, Java, Kalimantan. Cambodia: Bokor.

\section{B. violaceolabellum Seidenf.,}

1981, Nordic Journ. Bot. 1: 210; Newman et al., 2007, Checklist Vasc. Pl. Lao PDR: 857; Schuit. et al., 2008, Nord. Journ. Bot. 26: 272; Aver., 2012, Taiwania, 57, 2: 129.

Northeastern Laos, Houaphan province, Hem district, Nacoc village, 12 April 2015, N. T. Hiep, L. Averyanov, N. S. Khang et al., LA-VN 1457 (HNL, FOF, LE).

Creeping epiphyte and lithophyte. Primary and secondary broad-leaved evergreen open dry forests on rocky limestone, (500)1000-1200 m. Fl. March May. Not common.

Vietnam. Laos: Houaphan, Louangphrabang.

B. xylophyllum Par. et Rchb. f.,

1874, Trans. Linn. Soc. London 30: 151; Newman et al., 2007, Checklist Vasc. P1. Lao PDR: 257; Schuit. et al., 2008, Nord. Journ. Bot. 26: 272.

Central Laos, Vientiane province, Vang Vieng district, close vicinities of Vang Vieng town, 13 December 2015, L. Averyanov, T. Maisak, AL 203 (LE).

Creeping epiphyte and lithophyte. Primary and secondary evergreen broad-leaved forests on rocky limestone, 350-500 m. Fl. December - February. Rare.

NE. India, Bhutan, Myanmar, Thailand, Vietnam. Laos: Vientiane.

Calanthe $\mathrm{R}$. Br.

${ }^{\mathrm{L}}$ C. alismifolia Lindl.,

1854, Fol. Orch. Calanthe: 8.

Northeastern Laos, Houaphan province, Viengxay district, Vieng Xai village, 7 April 2015, N. T. Hiep, L. Averyanov, N. S. Khang et al., LA-VN 1116 (HNL, FOF, LE).

Terrestrial herb. Primary and secondary broadleaved evergreen dry forests on rocky limestone, commonly in shady humid depressions, 800-950 m. Fl. January - February, July - August. Locally common.

NE. India, Bhutan, Japan, China, Taiwan, Vietnam. Laos: Houaphan.

\section{C. cardioglossa Schltr.,}

1906, Repert. Spec. Nov. Regni Veg. 2: 85; Aver., 2013, Turczaninowia 16, 4: 30.
Southern Cambodia, Kampot province, Bokor National park, 16 December 2014, E. Konstantinov, $K-363$ (LE).

Lithophytic and terrestrial herb on rocky ground. Primary and secondary dense humid and cloud evergreen broad-leaved forests, often along streams, 600-1000 m. Fl. January, October - December. Locally common. Ornamental.

Thailand, Laos, Vietnam. Cambodia: Kampot.

\section{C. lyroglossa Rchb. f.,}

1878, Otia Bot. Hamburg.: 53; Newman et al., 2007, Checklist Vasc. Pl. Lao PDR: 257; Schuit. et al., 2008, Nord. Journ. Bot. 26: 272.

= Calanthe nephroidea Gagnep., 1951, Bull. Mus. Natl. Hist. Nat. 2, 22: 625.

Central Laos, Khammouan province, Nakai district, Ban Theung commune, Nakai Plateau, 19 January 2015, P. Thomas, R. Timmins, s.n. (LE photo).

Terrestrial herb. Primary and secondary humid evergreen, broad-leaved and coniferous forests, commonly in wet shady depressions growing on decaying timber, 900-1000 m. Fl. December February. Not common.

Myanmar, Taiwan, Thailand, Cambodia, Vietnam, Malacca Peninsula, Malaysia, Philippines. Laos: Champasak, Khammouan.

\section{C. odora Griff.}

1851, Not. Pl. Asiat. 3: 365; Newman et al., 2007, Checklist Vasc. P1. Lao PDR: 257; Schuit. et al., 2008, Nord. Journ. Bot. 26: 272.

$=$ Calanthe angusta Lindl., 1855, Fol. Orchid. 6: 7 .

Central Laos, Xiangkhouang province, Kham district, Huad village, 4 April 2015, N. T. Hiep, L. Averyanov, N. S. Khang et al., LA-VN 1060 (HNL, FOF, LE). Northeastern Laos, Houaphan province, Hem district, Khon Ngua village, 15 April 2015, N. T. Hiep, L. Averyanov, N. S. Khang e al., LAVN 1483 (HNL, FOF, LE). Northeastern Laos, Houaphan province, Hem district, Na Puok village, 11 April 2015, N. T. Hiep, L. Averyanov, N. S. Khang et al., LA-VN 1356 (HNL, FOF, LE). Northeastern Laos, Houaphan province, Hem district, Nacoc village, 12 April 2015, N. T. Hiep, L. Averyanov, N. S. Khang et al., LA-VN 1427 (HNL, FOF, LE). Northeastern Laos, Houaphan province, Viengxay district, Xieng Luong village, 8 April 2015, N. T. Hiep, L. Averyanov, N. S. Khang et al., LA-VN 1171 (HNL, FOF, LE).

Terrestrial and lithophytic herb. Primary and secondary broad-leaved evergreen and 
semideciduous dry forests on rocky limestone, commonly in humid shady places, 600-1400 m. Fl. April - June. Locally common. Ornamental.

NE. India, China, Thailand, Cambodia, Vietnam. Laos: Houaphan, Xiangkhouang.

${ }^{\mathrm{L}}$ C. sylvatica (Thouars) Lindl.,

1833, Gen. Sp. Orch. Pl.: 250.

Fig. 4D, E.

Central Laos, Khammouan province, Nakai district, Ban Theung commune, Nakai Plateau, 19 January 2015, P. Thomas, R. Timmins, s.n. (LE photo).

Terrestrial herb. Primary evergreen broad-leaved, mixed and coniferous forests, commonly in shady humid places, 800-900 m. Fl. December - January. Not common. Ornamental.

Africa, Madagascar, Sri Lanka, India, Bhutan, Nepal, Myanmar, China, Japan, Thailand, Malaysia, Indonesia. Laos: Khammouan.

Callostylis Blume

C. rigida Blume

1825, Bijdr.: 340; Aver., 2013, Turczaninowia 16, 4: 30 .

$=$ Eria discolor Lindl., 1858, Journ. Proc. Linn. Soc., Bot. 3: 51; Newman et al., 2007, Checklist Vasc. Pl. Lao PDR: 267; Schuit. et al., 2008, Nord. Journ. Bot. 26: 290.

$=$ Tylostylis discolor (Lind1.) Hook. f., 1895, Ann. Roy. Bot. Gard. (Calcutta) 5: 22.

Central Laos, Xiangkhouang province, Peak district, Khoang village, 3 April 2015, N. T. Hiep, L. Averyanov, N. S. Khang et al., LA-VN 975 (HNL, FOF, LE). Northeastern Laos, Houaphan province, Hem district, Na Puok village, 11 April 2015, N. T. Hiep, L. Averyanov, N. S. Khang et al., LA-VN 1332 (HNL, FOF, LE). Northeastern Laos, Houaphan province, Hem district, Nacoc village, 12 April 2015, N. T. Hiep, L. Averyanov, N. S. Khang et al., LA-VN 1393 (HNL, FOF, LE). Northeastern Laos, Houaphan province, Xon district, Nong Hua Pa Noi village, 11 April 2015, N. T. Hiep, L. Averyanov, N. S. Khang et al., $L A-V N 1231$ (HNL, FOF, LE).

Creeping epiphyte and occasionally lithophyte. Primary and secondary broad-leaved evergreen forests on any kind of soil, 800-1450 m. Fl. November - February. Very common, particularly on rocky limestone.

NE. India, Nepal, Thailand, Vietnam, Malacca Peninsula, Sumatra, Java, Borneo. Laos: Bolikhamxai, Champasak, Houaphan, Khammouan, Louang Namtha, Phongsali, Vientiane, Xiangkhouang.
Cheirostylis Blume

${ }^{\mathrm{L}} \boldsymbol{C}$. pusilla Lindl.,

1840, Gen. Sp. Orch. P1.: 489; Aver., 2015, Wulfenia 22: 150.

Northeastern Laos, Houaphan province, Hem district, Na Puok village, 11 April 2015, N. T. Hiep, L. Averyanov, N. S. Khang et al., LA-VN 1335 (HNL, FOF, LE).

Miniature creeping lithophyte. Primary and secondary broad-leaved evergreen dry forests on rocky limestone, 1050-1150 m. Fl. September - October. Rare.

NE. India, SW. China (S. Yunnan), Thailand, Vietnam, Malacca Peninsula.

The early record of this species for Laos (Averyanov, 2013: 32, fig. 1, I - E. Konstantinov et al., 14bOR-L-GPS018) belongs to another, not yet described species.

\section{Cleisostoma Blume}

C. arietinum (Rchb. f.) Garay,

1972, Bot. Mus. Leafl. 23: 169; Newman et al., 2007, Checklist Vasc. P1. Lao PDR: 258; Schuit. et al., 2008, Nord. Journ. Bot. 26: 274.

Central Laos, Xiangkhouang province, Kham district, Huad village, 4 April 2015, N. T. Hiep, L. Averyanov, N. S. Khang et al., LA-VN 1081 (HNL, FOF, LE). Central Laos, Xiangkhouang province, Peak district, Khoang village, 3 April 2015, N. T. Hiep, L. Averyanov, N. S. Khang et al., LA-VN 996 (HNL, FOF, LE). Northeastern Laos, Houaphan province, Hem district, Khon Ngua village, 15 April 2015, N. T. Hiep, L. Averyanov, N. S. Khang et al., $L A-V N 1500$ (HNL, FOF, LE). Northeastern Laos, Houaphan province, Hem district, Na Puok village, 11 April 2015, N. T. Hiep, L. Averyanov, N. S. Khang et al., LA-VN 1305 (HNL, FOF, LE). Northeastern Laos, Houaphan province, Hem district, Nacoc village, 12 April 2015, N. T. Hiep, L. Averyanov, N. S. Khang et al., LA-VN 1428 (HNL, FOF, LE).

Epiphyte. Primary and secondary broad-leaved evergreen and deciduous dry open forests on any kind of soil, 700-1450 m. Fl. January, March April. Common, particularly in open dipterocarp and limestone forests.

NE. India, Myanmar, Thailand, Cambodia, Vietnam, Malacca Peninsula. Laos: Champasak, Houaphan, Xiangkhouang.

\section{C. birmanicum (Schltr.) Garay}

1972, Bot. Mus. Leafl. 23: 170; Newman et al., 2007, Checklist Vasc. P1. Lao PDR: 258; Schuit. et al., 2008, Nord. Journ. Bot. 26: 274. 
Central Laos, Xiangkhouang province, Kham district, Huad village, 4 April 2015, N. T. Hiep, L. Averyanov, N. S. Khang et al., LA-VN 1082 (HNL, FOF, LE).

Epiphyte. Primary and secondary broad-leaved evergreen and semideciduous dry forests on any kind of soil, 1200-1400 m. Fl. June - July. Occasional.

Myanmar, China, Thailand, Vietnam. Laos: Champasak, Khammouan, Xiangkhouang.

\section{C. fuerstenbergianum Kraenzl.}

1909, Repert. Spec. Nov. Regni Veg. 7: 39; Newman et al., 2007, Checklist Vasc. Pl. Lao PDR: 259; Schuit. et al., 2008, Nord. Journ. Bot. 26: 275; Aver., 2013, Turczaninowia 16, 4: 33.

Central Laos, Khammouan province, Lak Sao town area, 31 October 2013, E. Konstantinov, $K$-220 (LE). Central Laos, Khammouan province, Thakhek town area, Pha In Cave, 3 November 2013, E. Konstantinov, K-234 (LE). Central Laos, Xiangkhouang province, Peak district, Oran village, 2 April 2015, N. T. Hiep, L. Averyanov, N. S. Khang et al., LA-VN 926 (HNL, FOF, LE).

Epiphyte. Primary and secondary evergreen and semideciduous broad-leaved open dipterocarp and limestone forests, particularly on hill and mountain tops, 100-1240 m. Fl. June - December. Not rare.

S. China, Thailand, Cambodia, Vietnam. Laos: Attapu, Bolikhamxai, Champasak, Khammouan, Louangphrabang, Phongsali, Vientiane, Xiangkhouang.

${ }^{\mathrm{L}}$ C. melanorachis Aver. et Averyanova, 2006, Komarovia 4: 8.

$4 \mathrm{~F}, \mathrm{G}$.

Northeastern Laos, Houaphan province, Hem district, Na Puok village, 11 April 2015, N. T. Hiep, L. Averyanov, N. S. Khang et al., LA-VN 1320 (HNL, FOF, LE). Northeastern Laos, Houaphan province, Hem district, Nacoc village, 12 April 2015, N. T. Hiep, L. Averyanov, N. S. Khang et al., LA-VN 1429 (HNL, FOF, LE). Northeastern Laos, Houaphan province, Viengxay district, Xieng Luong village, 8 April 2015, N. T. Hiep, L. Averyanov, N. S. Khang et al., LA-VN 1176 (HNL, FOF, LE).

Epiphyte. Primary and secondary broad-leaved evergreen dry forests on rocky limestone, commonly on hill and mountain tops, 600-1200 m. Fl. March - May. Locally common.

N. Vietnam. Laos: Houaphan.

C. simondii (Gagnep.) Seidenf., 1975, Dansk Bot. Ark. 29, 3: 66; Newman et al.,
2007, Checklist Vasc. Pl. Lao PDR: 259; Schuit. et al., 2008, Nord. Journ. Bot. 26: 275; Aver., 2013, Turczaninowia 16, 4: 33.

Central Laos, Xiangkhouang province, Peak district, Oran village, 2 April 2015, N. T. Hiep, L. Averyanov, N. S. Khang et al., LA-VN 924 (HNL, FOF, LE). Central Laos, Xiangkhouang province, Peak district, Khoang village, 3 April 2015, N. T. Hiep, L. Averyanov, N. S. Khang et al., LA-VN 987 (HNL, FOF, LE).

Epiphyte. Primary and secondary broad-leaved evergreen and deciduous dry forests on any kind of soil, often open limestone forests, 1200-1850 m. Fl. October - December. Occasional.

NE. India, China, Thailand, Vietnam. Laos: Champasak, Khammouan, Louangphrabang, Xiangkhouang.

${ }^{\mathrm{L}}$ C. subulatum Blume,

1825, Bijdr.: 363; Aver., 2015, Taiwania 60, 3: 115.

Central Laos, Xiangkhouang province, Peak district, Khoang village, 3 April 2015, N. T. Hiep, $L$. Averyanov, N. S. Khang et al., LA-VN 977 (HNL, FOF, LE). Northeastern Laos, Houaphan province, Hem district, Nacoc village, 12 April 2015, N. T. Hiep, L. Averyanov, N. S. Khang et al., LA-VN 1399 (HNL, FOF, LE).

Epiphyte and occasionally lithophyte. Primary and secondary broad-leaved evergreen dry forests on rocky limestone, 1000-1450 m. Fl. December January, March - July. Locally common.

NE. India, Bhutan, S. Myanmar, Thailand, Vietnam, Cambodia, Malacca Peninsula, Indonesia, Philippines. Laos: Houaphan, Xiangkhouang.

${ }^{\mathrm{L}}$ C. williamsonii (Rchb. f.) Garay, 1972, Bot. Mus. Leafl. Harv. Univ. 23, 4: 176.

Central Laos, Xiangkhouang province, Peak district, Khoang village, 3 April 2015, N. T. Hiep, $L$. Averyanov, N. S. Khang et al., LA-VN 960 (HNL, FOF, LE). Northeastern Laos, Houaphan province, Viengxay district, Xieng Luong village, 8 April 2015, N. T. Hiep, L. Averyanov, N. S. Khang et al., LA-VN 1197 (HNL, FOF, LE). Northeastern Laos, Houaphan province, Xon district, Nong Hua Pa Noi village, 11 April 2015, N. T. Hiep, L. Averyanov, N. S. Khang et al., LA-VN 1290 (HNL, FOF, LE).

Epiphyte. Primary and secondary broad-leaved evergreen dry forests on rocky limestone, 600-1450 m. Fl. April - May. Locally common.

NE. India, Bhutan, Myanmar, Thailand, S. China, Vietnam, Malacca Peninsula, Sumatra, Java, Kalimantan. Laos: Houaphan, Xiangkhouang. 
Coelogyne Lindl.

C. assamica Linden et Rchb.f.,

1857, Allg. Gartenzeitung 25: 403; Newman et al., 2007, Checklist Vasc. P1. Lao PDR: 259; Schuit. et al., 2008, Nord. Journ. Bot. 26: 275.

$=C$. siamensis Rolfe, 1914, Bull. Misc. Inform. Kew 1914: 373.

$=C$. annamensis Rolfe, 1914, Bull. Misc. Inform. Kew 1914: 211.

$=$ C. dalatensis Gagnep., 1930, Bull. Mus. Natl.

Hist. Nat. 2, 2: 423.

= Cymbidium evrardii Guillaumin, 1930, Bull.

Soc. Bot. France 77: 339.

= Coelogyne saigonensis Gagnep., 1938, Bull. Mus. Natl. Hist. Nat. 2, 10: 435.

= C. dichroantha Gagnep., 1950, Bull. Mus. Natl. Hist. Nat. 2I, 22: 506.

Southwestern Laos, Champasak province, Bolaven Plateau, Tad Gneuang Waterfall, 9 December 2014, E. Konstantinov, K-357 (LE).

Epiphyte. Primary and secondary evergreen broad-leaved forests, 1000-1200 m. Fl. November December. Not common. Ornamental.

NE. India, Bhutan, SW. China (Yunnan), Thailand, Vietnam. Laos: Champasak.

\section{C. calcicola Kerr,}

1933, Journ. Siam Soc., Nat. Hist. Suppl. 9: 233; Newman et al., 2007, Checklist Vasc. Pl. Lao PDR: 259; Schuit. et al., 2008, Nord. Journ. Bot. 26: 276.

Central Laos, Xiangkhouang province, Kham district, Huad village, 4 April 2015, N. T. Hiep, L. Averyanov, N. S. Khang et al., LA-VN 1068 (HNL, FOF, LE). Northeastern Laos, Houaphan province, Hem district, Nacoc village, 12 April 2015, N. T. Hiep, L. Averyanov, N. S. Khang et al., LA-VN 1447 (HNL, FOF, LE).

Epiphyte and lithophyte. Primary and secondary broad-leaved evergreen and semideciduous dry forests on rocky limestone, commonly on mountain tops, 1000-1500 m. Fl. April - May, July. Locally common. Ornamental.

Myanmar, SW. China (Yunnan), Vietnam. Laos: Champasak, Houaphan, Xiangkhouang.

\section{C. eberhardtii Gagnep.,} 423.

1930, Bull. Mus. Hist. Nat. (Paris) 2 ser. 2, 4:

Southwestern Laos, Champasak province, Pakse district, Bolaven Plateau, road Paksong - Ban Houay Kong, 26 November 2015, E. Konstantinov, T. Vongsa, K-390 (LE).

Epiphyte. Primary evergreen broad-leaved, mixed and coniferous humid forests preferably on granite and gneiss, 1100-1200 m. Fl. September October. Not common. Ornamental.

S. Vietnam. Laos: Champasak.

C. flaccida Lind1.,

1830, Gen. Sp. Orch. Pl.: 39; Newman et al., 2007, Checklist Vasc. Pl. Lao PDR: 259; Schuit. et al., 2008, Nord. Journ. Bot. 26: 276; Aver., 2013, Turczaninowia 16, 4: 33.

= Coelogyne lactea Rchb. f., 1885, Gard. Chron. n.s., 1885, 1: 692 .

Central Laos, Xiangkhouang province, Peak district, Khoang village, 3 April 2015, N. T. Hiep, L. Averyanov, N. S. Khang et al., LA-VN 995 (HNL, FOF, LE). Northeastern Laos, Houaphan province, Hem district, Na Puok village, 11 April 2015, N. T. Hiep, L. Averyanov, N. S. Khang et al., LA-VN 1328 (HNL, FOF, LE).

Lithophyte and occasionally epiphyte. Primary and secondary broad-leaved evergreen dry forests on rocky limestone, often on exposed rocks, 5001750 m. Fl. March - April. Locally common. Ornamental.

NE. India, Nepal, Myanmar, Thailand, China. Laos: Houaphan, Vientiane, Xiangkhouang.

${ }^{\mathrm{L}}$ C. leucantha W. W. Sm.,

1921, Notes Roy. Bot. Gard. Edinburgh 13: 198; Aver. et al., 2015, Wulfenia 22: 152.

Central Laos, Xiangkhouang province, Peak district, Oran village, 2 April 2015, N. T. Hiep, L. Averyanov, N. S. Khang et al., LA-VN 931 / TM 1221 (HNL, FOF, LE).

Creeping epiphyte. Primary broad-leaved mixed and coniferous evergreen humid forests on shale sandstone, commonly on tall mossy trees near mountain tops, 1750-1850 m. Fl. March - May (June). Locally common.

N. Myanmar, S. China, N. Vietnam. Laos: Xiangkhouang.

Recently also found in Vietnam (Averyanov et al., 2015).

${ }^{\mathrm{L}}$ C. ovalis Lindl.,

1838, Bot. Reg. 24, Misc.: 91.

Northeastern Laos, Houaphan province, Hem district, Nacoc village, 12 April 2015, N.T. Hiep, L. Averyanov, N.S. Khang et al., LA-VN 1462 (HNL, FOF, LE).

Creeping epiphyte. Primary and secondary broad-leaved evergreen open dry forests on rocky limestone near mountain tops, 1000-1200 m. Fl. August - November. Occasional. 
NE. India, Bhutan, Nepal, Myanmar, S. China, Vietnam. Laos: Houaphan.

\section{C. trinervis Lindl.,}

1830, Gen. Sp. Orch. Pl.: 41; Newman et al., 2007, Checklist Vasc. Pl. Lao PDR: 260; Schuit. et al., 2008, Nord. Journ. Bot. 26: 277.

Northeastern Laos, Houaphan province, Hem district, Nacoc village, 12 April 2015, N. T. Hiep, L. Averyanov, N. S. Khang et al., LA-VN 1395 (HNL, FOF, LE). Northeastern Laos, Houaphan province, Xon district, Nong Hua Pa Noi village, 11 April 2015, N. T. Hiep, L. Averyanov, N. S. Khang et al., $L A-V N 1245$ (HNL, FOF, LE).

Creeping epiphyte. Primary and secondary broad-leaved evergreen open dry dipterocarp and limestone forests, 350-1200 m. Fl. September - December. Locally common. Ornamental.

Myanmar, Thailand, Vietnam, Cambodia, Malacca Peninsula, Sumatra, Java, Lesser Sunda Islands. Laos: Attapu, Bolikhamxai, Champasak, Houaphan, Khammouan, Savannakhet, Vientiane, Xaisomboun.

\section{C. viscosa Rchb. f.,}

1856, Allg. Gartenzeitung 24: 218; Newman et al., 2007, Checklist Vasc. P1. Lao PDR: 260; Schuit. et al., 2008, Nord. Journ. Bot. 26: 277; Aver., 2013, Turczaninowia 16, 4: 33.

Central Laos, Xiangkhouang province, Kham district, Huad village, 4 April 2015, N. T. Hiep, L. Averyanov, N. S. Khang et al., LA-VN 1052 (HNL, FOF, LE). Northeastern Laos, Houaphan province, Hem district, Na Puok village, 11 April 2015, N. T. Hiep, L. Averyanov, N. S. Khang et al., LA-VN 1327 (HNL, FOF, LE). Northeastern Laos, Houaphan province, Hem district, Nacoc village, 12 April 2015, N. T. Hiep, L. Averyanov, N. S. Khang et al., $L A-V N 1448$ (HNL, FOF, LE).

Epiphyte. Primary and secondary broad-leaved evergreen and semideciduous open dry forests on rocky limestone, particularly on hill and mountain tops and along streams, 300-1400 m. Fl. DecemberFebruary. Locally common.

NE. India, Myanmar, S. China, Thailand, Vietnam, Malacca Peninsula. Laos: Bolikhamxai, Champasak, Houaphan, Khammouan, Phongsali, Vientiane, Xaisomboun, Xiangkhouang.

\section{Corymborkis Thouars}

C. veratrifolia (Reinw.) Blume,

1859, Coll. Orch.: 125; Newman et al., 2007, Checklist Vasc. P1. Lao PDR: 260; Schuit. et al.,
2008, Nord. Journ. Bot. 26: 277; Aver., 2013, Turczaninowia 16, 4: 33 .

Northeastern Laos, Houaphan province, Viengxay district, Xieng Luong village, 8 April 2015, $N$. T. Hiep, L. Averyanov, N. S. Khang et al., LA-VN 1203 (HNL, FOF, LE).

Terrestrial semi-woody herb. Primary and secondary broad-leaved evergreen dry forests on rocky limestone, usually in humid shady depressions and stream valleys with rich soil, 300-800 m. Fl. May July. Occasional.

Tropical Asia and Australia, tropical Pacific islands. Laos: Bolikhamxai, Houaphan, Louang Namtha, Saravan, Vientiane.

\section{Cymbidium Sw.}

C. aloifolium (L.) Sw.,

1799, Nova Acta Regiae Soc. Sci. Upsal. 6: 73; Seidenf., 1992, Opera Bot.: 338; Newman et al., 2007, Checklist Vasc. Pl. Lao PDR: 260; Schuit. et al., 2008, Nord. Journ. Bot. 26: 278; Aver., 2013, Turczaninowia 16, 4: 33.

Southern Cambodia, to the N of Sihanoukville town, Kaoh Rong Island in Siam Gulf, 19 November 2011, T. Maisak, M. Telepova, L. Osinovetz, 845 (LE). Central Laos, Xiangkhouang province, Kham district, Huad village, 4 April 2015, N. T. Hiep, L. Averyanov, N. S. Khang et al., LA-VN 1072 (HNL, FOF, LE). Northeastern Laos, Houaphan province, Viengxay district, Xieng Luong village, 8 April 2015, N. T. Hiep, L. Averyanov, N. S. Khang et al., LA-VN 1179 (HNL, FOF, LE).

Epiphyte and lithophyte. Occurs in any kind of plant communities, 20-1400 m. Fl. March - May. One of the most common orchids in Cambodia and Laos.

Sri Lanka, India, Andaman Islands, Nepal, Bangladesh, China, Myanmar, Thailand, Vietnam, Malacca Peninsula, Java. Cambodia: Kampong Chhnang, Sihanoukville. Laos: Attapu, Houaphan, Khammouan, Louangphrabang, Vientiane, Xaignabouri, Xiangkhouang.

\section{Dendrobium Sw.}

D. aphyllum (Roxb.) C. E. C. Fisch.,

1928, Fl. Madras: 1416; Newman et al., 2007, Checklist Vasc. P1. Lao PDR: 262; Schuit. et al., 2008, Nord. Journ. Bot. 26: 279.

$\equiv$ Limodorum aphyllum Roxb., 1795, Pl. Coromandel 1: 34.

= Dendrobium pierardii Hook., 1822, Exot. Fl. 1, t. 9 .

=D. oxyphyllum Gagnep., 1950, Bull. Mus. Natl. Hist. Nat. 2, 21: 743. 
Central Laos, Xiangkhouang province, Kham district, Huad village, 4 April 2015, N. T. Hiep, $L$. Averyanov, N. S. Khang et al., LA-VN 1049 (HNL, FOF, LE). Central Laos, Xiangkhouang province, Kham district, Huad village, 4 April 2015, N. T. Hiep, L. Averyanov, N. S. Khang et al., LA-VN 1080 (HNL, FOF, LE). Central Laos, Xiangkhouang province, Peak district, Khoang village, 3 April 2015, N. T. Hiep, L. Averyanov, N. S. Khang et al., LA-VN 957 (HNL, FOF, LE). Northeastern Laos, Houaphan province, Hem district, Khon Ngua village, 15 April 2015, N. T. Hiep, L. Averyanov, N. S. Khang et al., $L A-V N 1465$ (HNL, FOF, LE). Northeastern Laos, Houaphan province, Hem district, Na Puok village, 11 April 2015, N. T. Hiep, L. Averyanov, N. S. Khang et al., LA-VN 1323 (HNL, FOF, LE). Northeastern Laos, Houaphan province, Viengxay district, Xieng Luong village, 8 April 2015, N. T. Hiep, L. Averyanov, N. S. Khang et al., LA-VN 1177 (HNL, FOF, LE). Northeastern Laos, Houaphan province, Xon district, Nong Hua Pa Noi village, 11 April 2015, N. T. Hiep, L. Averyanov, N. S. Khang et al., LA-VN 1269 (HNL, FOF, LE).

Epiphyte or occasionally lithophyte. Primary and secondary broad-leaved and mixed evergreen and semideciduous dry open forests on any kind of soil, preferably on rocky limestone, $150-1400 \mathrm{~m}$. Fl. February - July. Occasional. Ornamental.

India, Nepal, Bhutan, Myanmar, China, Thailand, Cambodia, Vietnam, Malacca Peninsula. Laos: Bolikhamxai, Champasak, Houaphan, Khammouan, Louang Namtha, Louangphrabang, Phongsali, Vientiane, Xaignabouri, Xiangkhouang.

\section{D. christyanum Rchb. f.,}

1882, Gard. Chron. 1882 (1): 178; Newman et al., 2007, Checklist Vasc. Pl. Lao PDR: 262; Schuit. et al., 2008, Nord. Journ. Bot. 26: 280.

Central Laos, Xiangkhouang province, Peak district, Oran village, 2 April 2015, N. T. Hiep, L. Averyanov, N. S. Khang et al., LA-VN 922 (HNL, FOF, LE).

Epiphyte. Primary and secondary broad-leaved evergreen humid mossy forests on shale and sandstone on mountain tops, 1200-1900 m. Fl. May July. Rare. Ornamental.

Thailand, Vietnam. Laos: Champasak, Xiangkhouang.

D. chrysanthum Wall.,

1830, Bot. Reg. 15, t. 1299; Newman et al., 2007, Checklist Vasc. Pl. Lao PDR: 262; Schuit. et al., 2008, Nord. Journ. Bot. 26: 280.
Northeastern Laos, Houaphan province, Hem district, Na Puok village, 11 April 2015, N. T. Hiep, L. Averyanov, N. S. Khang et al., LA-VN 1304 (HNL, FOF, LE).

Lithophyte and epiphyte. Primary and secondary broad-leaved evergreen dry open forests preferably on rocky limestone, particularly on mossy exposed rocks on hill and mountain tops, $1050-1700 \mathrm{~m}$. Fl. April - September.

India, Nepal, Bhutan, Myanmar, China, Thailand, Vietnam. Laos: Champasak, Houaphan, Savannakhet, Xaisomboun, Xiangkhouang.

\section{D. chryseum Rolfe,}

1888, Gard. Chron. 3, 3: 233; Newman et al., 2007, Checklist Vasc. P1. Lao PDR: 262; Schuit. et al., 2008, Nord. Journ. Bot. 26: 280.

Central Laos, Xiangkhouang province, Kham district, Huad village, 4 April 2015, N. T. Hiep, L. Averyanov, N. S. Khang et al., LA-VN 1030 (HNL, FOF, LE).

Epiphyte. Primary and secondary broad-leaved evergreen and semideciduous dry forests preferably on rocky limestone, 1100-1400 m. Fl. March April. Rare. Ornamental.

India, Nepal, Bhutan, Myanmar, China, Taiwan, Thailand, Vietnam. Laos: Vientiane, Xiangkhouang.

\section{D. chrysotoxum Lindl.,}

1847, Bot. Reg. 33, t. 19; Newman et al., 2007, Checklist Vasc. Pl. Lao PDR: 262; Schuit. et al., 2008, Nord. Journ. Bot. 26: 280.

Central Laos, Xiangkhouang province, Kham district, Huad village, 4 April 2015, N. T. Hiep, L. Averyanov, N. S. Khang et al., LA-VN 1092 (HNL, FOF, LE). Central Laos, Xiangkhouang province, Peak district, Khoang village, 3 April 2015, N. T. Hiep, L. Averyanov, N. S. Khang et al., LA-VN 985 (HNL, FOF, LE). Northeastern Laos, Houaphan province, Hem district, Na Puok village, 11 April 2015, N. T. Hiep, L. Averyanov, N. S. Khang et al., $L A-V N 1295$ (HNL, FOF, LE).

Epiphyte and occasionally lithophyte. Primary and secondary broad-leaved evergreen and semideciduous dry forests preferably on rocky limestone, particularly on mountain tops, 300-1400 m. Fl. January - May. Locally common. Ornamental.

NE. India, Nepal, Bhutan, Myanmar, China, Thailand, Vietnam. Laos: Bolikhamxai, Champasak, Houaphan, Khammouan, Phongsali, Savannakhet, Vientiane, Xaisomboun, Xiangkhouang. 
D. concinnum Miq.,

1859, Fl. Ned. Ind. 3: 644; Seidenf., 1992, Opera Bot.: 252 .

Southern Cambodia, to the N of Sihanoukville town, Kaoh Rong Island in Siam Gulf, 29 November 2012, T. Maisak et al., 1047 (LE).

Epiphyte. Primary and secondary evergreen dry lowland forests on low hills, 50-100 m. Fl. March May. Locally common.

Myanmar, Thailand, Malacca Peninsula, Vietnam, Sumatra, Java, Kalimantan. Cambodia: Kampot, Sihanoukville.

D. crepidatum Lindl. et Paxton,

1850, Fl. Gard. 1: 63; Newman et al., 2007, Checklist Vasc. P1. Lao PDR: 262; Schuit. et al., 2008, Nord. Journ. Bot. 26: 281; Aver., 2013, Turczaninowia 16, 4: 34.

Northeastern Laos, Houaphan province, Hem district, Nacoc village, 12 April 2015, N. T. Hiep, L. Averyanov, N. S. Khang et al., LA-VN 1406 (HNL, FOF, LE).

Epiphyte. Primary and secondary broad-leaved evergreen and deciduous open dry forests on any kind of soil, preferably on rocky limestone, 500 1200 m. Fl. March - May. Not common. Ornamental.

India, Nepal, Bhutan, Myanmar, China, Thailand, Cambodia, Vietnam. Laos: Attapu, Bolikhamxai, Champasak, Houaphan, Khammouan, Phongsali, Vientiane.

D. dixanthum Rchb. f.,

1865, Gard. Chron. 1865: 674; Newman et al., 2007, Checklist Vasc. Pl. Lao PDR: 263; Schuit. et al., 2008, Nord. Journ. Bot. 26: 282; Aver., 2012, Taiwania, 57, 2: 130, fig. 6 .

Northeastern Laos, Houaphan province, Hem district, Nacoc village, 12 April 2015, N. T. Hiep, L. Averyanov, N. S. Khang et al., LA-VN 1415 (HNL, FOF, LE).

Epiphyte. Primary and secondary broad-leaved evergreen open dry forests on rocky limestone, commonly on hill and mountain tops, 1000-1200 m. Fl. March - April. Not common. Ornamental.

Myanmar, China, Thailand, Vietnam. Laos: Houaphan.

D. ellipsophyllum Tang et F. T. Wang,

1951, Acta Phytotax. Sin. 1: 81; Seidenf., 1992, Opera Bot.: 238.

Southern Cambodia, to the N of Sihanoukville town, Kaoh Rong Island in Siam Gulf, 20 November
2011, T. Maisak, L. Osinovetz, M. Telepova, 829

(LE). Southwestern Cambodia, Koh Kong province, Koh Rong Island, 2013, E. Konstantinov, Chulisov s.n. (LE).

Epiphyte. Primary and secondary evergreen broad-leaved dry lowland forests on low hills, 2-150 m. FL. April - June. Locally common.

Myanmar, S. China (SE. Yunnan), Thailand, Vietnam, Laos. Cambodia: Kampot, Sihanoukville, Stung Treng.

${ }^{\mathrm{L}}$ D. eriiflorum Griff.,

1851, Ic. Pl. Asiat. 3: 316.

Fig. 4H, I.

Southwestern Laos, Champasak province, Pakse district, Bolaven Plateau, road Paksong - Ban Houay Kong, 26 November 2015, E. Konstantinov, T. Vongsa, K-391 (LE).

Epiphyte. Primary and secondary broad-leaved evergreen and semideciduous forests, 1100-1200 m. Fl. October - December. Rare.

N. India, Nepal, Bhutan, Myanmar. Laos: Champasak.

${ }^{\mathrm{L}}$ D. falconeri Hook.,

1856, Bot. Mag. 82, t. 4944.

Central Laos, Xiangkhouang province, Peak district, Oran village, 1750-1850 m, 2 April 2015, N. T. Hiep, L. Averyanov, N. S. Khang et al., LA-VN 927 (HNL, FOF, LE).

Epiphyte. Primary and secondary broad-leaved evergreen dry forest on shale sandstone, commonly on mountain tops and ridge edges, 1700-1900 m. Fl. April - June. Locally common. Ornamental.

NE. India, Bhutan, China, Taiwan, Myanmar, N. Thailand, N. Vietnam. Laos: Xiangkhouang.

A great amount of this species specimens observed in Vietnamese street markets in 2014-2016 originated certainly from northern Laos where it may be still very common. In Vietnam, this species is extremely rare and stands on the verge of full extinction.

\section{D. fimbriatum Hook.,}

1823, Exot. Fl. 1, t. 71; Newman et al., 2007, Checklist Vasc. Pl. Lao PDR: 263; Schuit. et al., 2008, Nord. Journ. Bot. 26: 282.

Central Laos, Xiangkhouang province, Peak district, Khoang village, 3 April 2015, N. T. Hiep, L. Averyanov, N. S. Khang et al., LA-VN 942 (LE photo). Northeastern Laos, Houaphan province, Viengxay district, Vieng Xai village, 7 April 2015, $N$. T. Hiep, L. Averyanov, N. S. Khang et al., LA-VN 
1150 (HNL, FOF, LE). Northeastern Laos, Houaphan province, Viengxay district, Xieng Luong village, 8 April 2015, N. T. Hiep, L. Averyanov, N. S. Khang et al., LA-VN 1153 (HNL, FOF, LE).

Lithophyte and epiphyte. Primary and secondary broad-leaved evergreen and semideciduous forests on rocky limestone, commonly on mossy exposed rocks on hill and mountain tops, 350-1500 m. Fl. March - May. Not rare. Ornamental.

India, Nepal, Bhutan, Myanmar, China, Thailand, Vietnam. Laos: Champasak, Houaphan, Khammouan, Louang Namtha, Louangphrabang, Vientiane, Xiangkhouang.

\section{D. findleyanum Par. et Rchb.f.,}

1874, Trans. Linn. Soc. London 30: 149; Newman et al., 2007, Checklist Vasc. Pl. Lao PDR: 263; Schuit. et al., 2008, Nord. Journ. Bot. 26: 282; Aver., 2013, Turczaninowia 16, 4: 34.

Central Laos, Xiangkhouang province, Peak district, Khoang village, Pa Khao mt., 3 April 2015, N. T. Hiep, L. Averyanov, N. S. Khang et al., LA$V N 958$ (HNL, FOF, LE). Northeastern Laos, Houaphan province, Hem district, Nacoc village, 12 April 2015, N. T. Hiep, L. Averyanov, N. S. Khang et al., $L A-V N 1416$ (HNL, FOF, LE). Northeastern Laos, Houaphan province, Xon district, Nong Hua Pa Noi village, 11 April 2015, N. T. Hiep, L. Averyanov, N. S. Khang et al., LA-VN 1253 (HNL, FOF, LE).

Epiphyte. Primary and secondary broad-leaved and mixed evergreen dry forests preferably on rocky limestone, 700-1600 m. Fl. (January) March - May. Not common. Ornamental.

Myanmar, Thailand, Vietnam. Laos: Champasak, Houaphan, Louang Namtha, Vientiane, Xiangkhouang.

A great amount of this species specimens observed in Vietnamese street markets in 2014-2016 originated certainly from northern Laos where it may be still very common. In Vietnam, this species is extremely rare and stands on the verge of full extinction.

\section{D. gratiosissimum Rchb. f.,}

1865, Bot. Zeitung (Berlin) 23: 99; Newman et al., 2007, Checklist Vasc. P1. Lao PDR: 263; Schuit. et al., 2008, Nord. Journ. Bot. 26: 283; Aver., 2013, Turczaninowia 16, 4: 34.

Central Laos, Xiangkhouang province, Kham district, Huad village, 4 April 2015, N. T. Hiep, L. Averyanov, N. S. Khang et al., LA-VN 1075 (HNL, FOF, LE). Central Laos, Xiangkhouang province, Kham district, Huad village, 4 April 2015, N. T.
Hiep, L. Averyanov, N. S. Khang et al., LA-VN 1097 (HNL, FOF, LE). Northeastern Laos, Houaphan province, Xon district, Nong Hua Pa Noi village, 11 April 2015, N. T. Hiep, L. Averyanov, N. S. Khang et al., LA-VN 1250 (HNL, FOF, LE).

Epiphyte. Primary and secondary broad-leaved evergreen and semideciduous dry forests on any kind of soil preferably on rocky limestone, 10001400 m. Fl. March - May. Occasional. Ornamental.

NE. India, Myanmar, China, Thailand, Vietnam. Laos: Bolikhamxai, Champasak, Houaphan, Khammouan, Phongsali, Vientiane, Xiangkhouang.

\section{D. jenkinsii Wall.,}

1839, Bot. Reg. 25, t. 37; Newman et al., 2007, Checklist Vasc. Pl. Lao PDR: 264; Schuit. et al., 2008, Nord. Journ. Bot. 26: 284.

= D. marseillei Gagnep., 1934, Bull. Mus. Natl. Hist. Nat. 2, 6: 119.

Central Laos, Xiangkhouang province, Kham district, Huad village, 4 April 2015, N. T. Hiep, L. Averyanov, N. S. Khang et al., LA-VN 1055 (HNL, FOF, LE). Central Laos, Xiangkhouang province, Peak district, Khoang village, 3 April 2015, N. T. Hiep, L. Averyanov, N. S. Khang et al., LA-VN 994 (HNL, FOF, LE). Northeastern Laos, Houaphan province, Viengxay district, Vieng Xai village, 7 April 2015, N. T. Hiep, L. Averyanov, N. S. Khang et al., LA-VN 1104 (HNL, FOF, LE). Northeastern Laos, Houaphan province, Xon district, Nong Hua Pa Noi village, 11 April 2015, N. T. Hiep, L. Averyanov, N. S. Khang et al., LA-VN 1251 (HNL, FOF, LE).

Epiphyte. Primary and secondary broad-leaved evergreen humid forests on mountain tops, particularly on rocky limestone, 900-1450 m. Fl. March April. Locally common. Ornamental.

NE. India, Bhutan, Myanmar, China, Thailand, Vietnam. Laos: Houaphan, Khammouan, Phongsali, Xiangkhouang.

\section{D. lagarum Seidenf.,}

1985, Opera Bot. 83: 187; Newman et al., 2007, Checklist Vasc. Pl. Lao PDR: 264; Schuit. et al., 2008, Nord. Journ. Bot. 26: 284; Aver., 2010, Taiwania 55, 1: 4.

Central Laos, Khammouan province, Thakhek town area, Pha In Cave, E. Konstantinov, K-238 (LE).

Epiphyte. Primary and secondary evergreen broad-leaved forests on rocky limestone, 100-200 m. Fl. January. Not common.

Thailand, Vietnam. Laos: Champasak, Khammouan. 


\section{D. loddigesii Rolfe,}

1887, Gard. Chron. 3, 2: 155; Newman et al., 2007, Checklist Vasc. Pl. Lao PDR: 264; Schuit. et al., 2008, Nord. Journ. Bot. 26: 284.

Central Laos, Xiangkhouang province, Peak district, Khoang village, 3 April 2015, N. T. Hiep, L. Averyanov, N. S. Khang et al., LA-VN 955 (HNL, FOF, LE). Northeastern Laos, Houaphan province, Hem district, Na Puok village, 11 April 2015, N. T. Hiep, L. Averyanov, N. S. Khang et al., LA-VN 1317 (HNL, FOF, LE). Northeastern Laos, Houaphan province, Xon district, Nong Hua Pa Noi village, 11 April 2015, N. T. Hiep, L. Averyanov, N. S. Khang et al., LA-VN 1249 (HNL, FOF, LE).

Epiphyte at base of trees and lithophyte. Primary and secondary broad-leaved evergreen dry forests on rocky limestone, 1100-1450 m. Fl. March - May. Locally common. Ornamental.

India, Nepal, Bhutan, Myanmar, China, Thailand, Cambodia, Vietnam. Laos: Houaphan, Xiangkhouang.

\section{D. nobile Lindl.,}

1830, Gen. Sp. Orch. Pl.: 79; Newman et al., 2007, Checklist Vasc. P1. Lao PDR: 264; Schuit. et al., 2008, Nord. Journ. Bot. 26: 285.

= D. chlorostylum Gagnep., 1950, Bull. Mus. Natl. Hist. Nat. 2, 21: 739.

Central Laos, Xiangkhouang province, Kham district, Huad village, 4 April 2015, N. T. Hiep, L. Averyanov, N. S. Khang et al., LA-VN 1035 (HNL, FOF, LE). Central Laos, Xiangkhouang province, Peak district, Khoang village, 3 April 2015, N. T. Hiep, L. Averyanov, N. S. Khang et al., LA-VN 1000 (HNL, FOF, LE).

Epiphyte and occasionally lithophyte. Primary and secondary broad-leaved and coniferous evergreen and semideciduous dry forests on mountain tops preferably on rocky limestone, 1200-1450 m. Fl. March - May. Not rare. Ornamental.

India, Nepal, Bhutan, Myanmar, China, Taiwan, Thailand, Vietnam. Laos: Bolikhamxai, Saravan, Vientiane, Xaignabouri, Xaisomboun, Xiangkhouang.

\section{D. minusculum Aver., sp. nov.}

Described from northeastern Laos ("Xiangkhouang province, Peak district, Oran village, primary and secondary broad-leaved evergreen dry forest on shale sandstone slopes and along ridge edge at elevation 1750-1850 m a.s.1., clustering epiphyte on tall tree, rare"). Type ("2 April 2015, N. T. Hiep, L. Averyanov, N. S. Khang,
N. Q. Hieu, T. Maisak, Pheng Phengsintham, LA$V N$ 925") - LE (holotype), HNL, FOF (isotypes). Epitype - d-EXSICCATES OF VIETNAMESE FLORA 0253/LA-VN 925.

Fig. 5; 6 (d-EXSICCATES OF VIETNAMESE FLORA 0253/LA-VN 925).

Species epithet refers minuscule plant habit.

Perennial clustering sympodial miniature epiphytic herb. Rhizome very short, negligible. Stems densely clustering, pseudobulbous, erect, with (2)3-4(5) nodes, globose to ovoid and narrowly ovoid, (0.4)0.5-1.5(2) cm tall, (2.5)3-4(5) $\mathrm{mm}$ in diam., with (1)2-3(4) leaves, young stems glossy green, enveloped by white to light greenish hyaline leaf sheaths, old finely rough, dark green to brown, naked or with light brownish scarious sheath remains at nodes. Leaves, distichous, sessile, sheathed; sheath tubular, as long as internode, completely disintegrating or remaining in form of insignificant scarious, papyraceous remnants on second-year stems; leaf blade conduplicate, with prominent median vein, suberect or recurved, rather thin, herbaceous, oblong lanceolate, (0.6)1-2(2.2) cm long, (2)3-5(5.5) mm wide, obtuse, sometime shortly unequally bilobulate at apex. Inflorescence $1-2$, synanthous, arising from the apical part of young leafy stem, suberect, lax, many flowered raceme (1)1.5-5(6) cm tall; scape and rachis light green, straight, scape short (4)5-10(12) mm long, naked or with 1-2 small sterile bracts. Scape bracts and floral bracts, light greenish, narrowly triangular, straight, conduplicate, tapering and acute at apex, (1.5)24(4.5) mm long, (0.5)0.8-1(1.2) mm wide. Pedicel and ovary light green, glabrous, (1)1.2-1.6(1.8) $\mathrm{mm}$ long, bent down at apex; pedicel cylindric, ovary obconoid, slightly longitudinally grooved. Flowers nodding, not much widely opening, light greenish, lip apical half light green, (3.2)3.5-4.5(5) $\mathrm{mm}$ across. Sepals spreading, narrowly ovate to broadly lanceolate, obtuse, (2.8)3-3.4(3.6) $\mathrm{mm}$ long, (0.7)0.8-1(1.2) $\mathrm{mm}$ wide, lateral sepals at the base oblique broadening to $1.8-2 \mathrm{~mm}$ wide, forming short broad saccate mentum $0.8-1 \mathrm{~mm}$ long, $1.2-$ $1.5 \mathrm{~mm}$ wide. Petals spreading, oblong broadly lanceolate, obtuse, little shorter and narrower than sepals. Lip more or less movable, entire, narrowly ovate, (2.2)2.4-2.6(2.8) mm long, (1)1.2-1.4(1.6) $\mathrm{mm}$ wide, fleshy, glabrous, recurved, blunt to round at apex, with 2 low simple fleshy keels running from the lip base to the epichile. Column light green, very short, erect, stout, shortly cylindric, $0.5-0.6$ $\mathrm{mm}$ tall, $0.8-1 \mathrm{~mm}$ wide, at front with prominent wing-like purple stelidia $0.6-0.8 \mathrm{~mm}$ tall, exceeding 
operculum; stigma large, sub-circular, concave; rostellum in form of small, thin, truncate plate; column foot twice longer than the column proper, thick, concave, shortly cymbiform, $1.2-1.4 \mathrm{~mm}$ long, $0.8-1 \mathrm{~mm}$ wide, forward curved. Anther cap light yellow, simple, conical hemispheric, smooth, $0.3-0.4 \mathrm{~mm}$ tall and wide, at front with broad truncate beak. Pollinia 4, lens-shaped, in two pair, yellow, oblong ovoid, $0.2-0.25 \mathrm{~mm}$ long.
Miniature branch and trunk epiphyte. Primary montane evergreen, broad-leaved forests on shaly sandstone, 1750-1850 m. Fl. August - September. Rare.

Laos: Xiangkhouang. Endemic.

Morphologically new species is most close to Dendrobium caespitosum King et Pantl., D. mucronatum Seidenf. and D. porphyrochilum Lindl., from which differs in smaller uniform pale green flowers

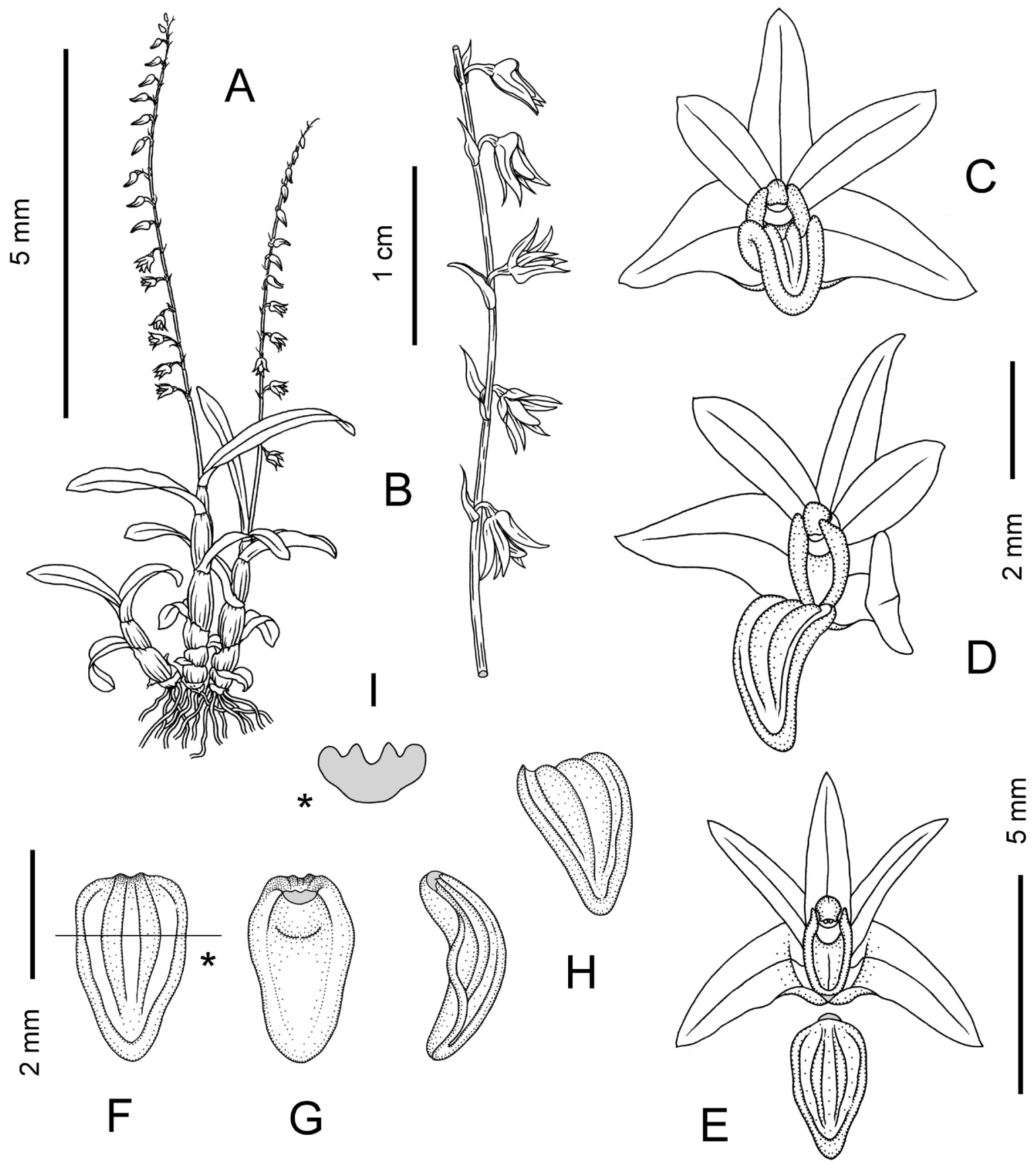

Fig. 5. New and rare orchids in the flora of Cambodia and Laos. Dendrobium minusculum Aver. A - Flowering plant. B - Portion of inflorescence. C, D - Flower, half side view. E - Flattened flower, frontal view. F - Lip, view from above. G - Lip, view from below. H - Lip, side and half side views. I - Transversal section of the lip along line marked in figure $\mathrm{F}$ by asterisk (*). All drawn from the type - LA-VN 925 by L. Averyanov and T. Maisak. 


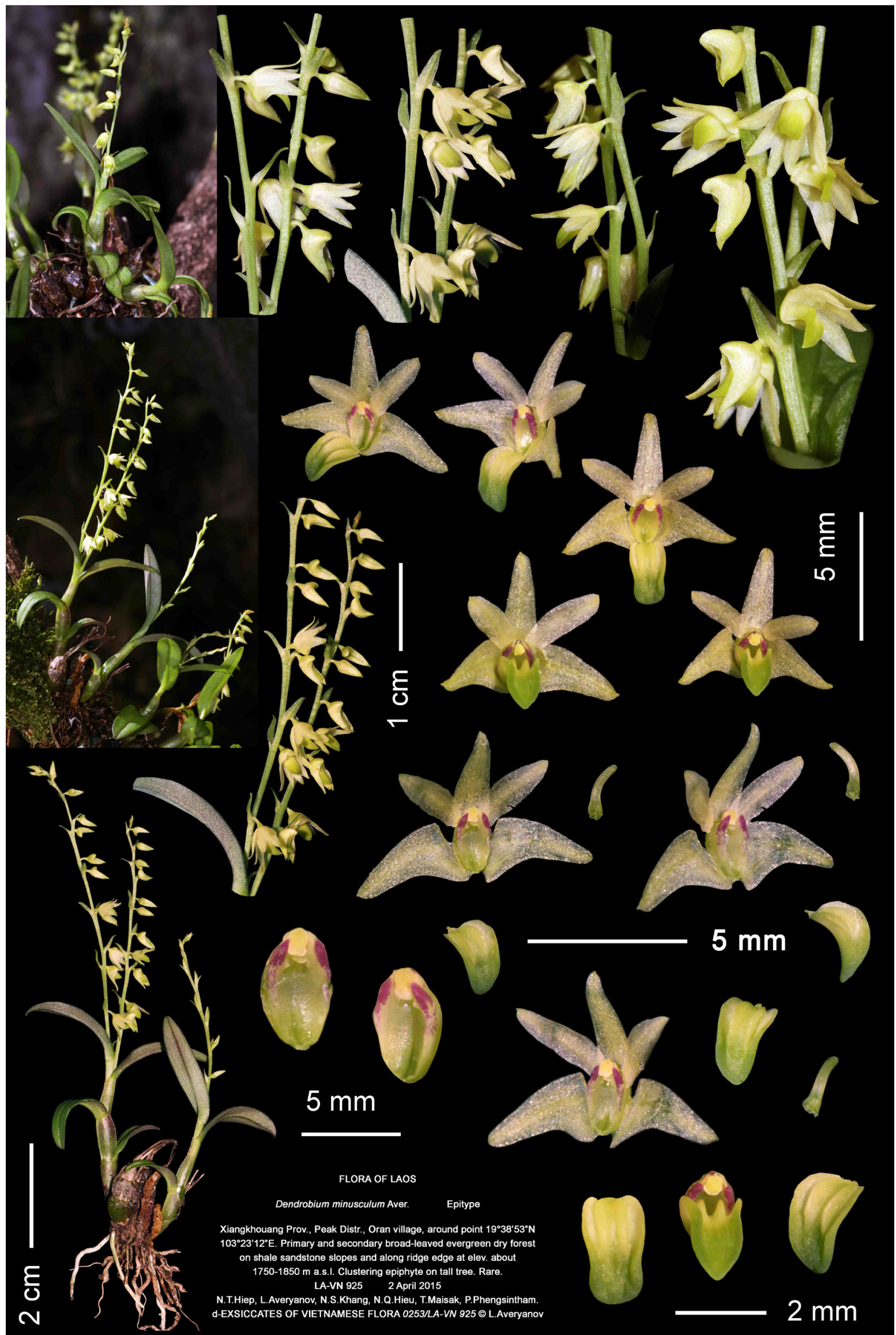

Fig. 6. New and rare orchids in the flora of Cambodia and Laos. Dendrobium minusculum Aver. Digital epitype - dEXSICCATES OF LAOTIAN FLORA 0253/LA-VN 925. Photos, design and image correction by L. Averyanov and T. Maisak. 
and very fleshy, strongly recurved lip with two fat keels separated by deep groove. This tiny epiphyte grows in canopies of tall trees, hence its occurrence is difficult to indicate in intact habitats. It was documented until now from lone locality.

${ }^{\mathrm{C}}$ D. pachyphyllum (Kuntze) Bakh. f., 1963, Blumea 12: 69.

Southwestern Cambodia, eastern part of Cardamom Mountains, Phnom Aural mountain, 23 November 2012, T. Maisak et al., 953a (LE).

Epiphyte. Primary and secondary evergreen and semideciduous dry open lowland frosts. Fl. March May. Not common.

NE. India, Myanmar, Thailand, Vietnam, Malacca Peninsula, Sumatra, Java, Kalimantan. Cambodia: border of Pursat and Kampong Speu provinces.

\section{D. parciflorum Lindl.,}

1858, Journ. Proc. Linn. Soc., Bot. 3: 4; Newman et al., 2007, Checklist Vasc. Pl. Lao PDR: 264; Schuit. et al., 2008, Nord. Journ. Bot. 26: 285.

Central Laos, Xiangkhouang province, Peak district, Khoang village, 3 April 2015, N. T. Hiep, L. Averyanov, N. S. Khang et al., LA-VN 991 (HNL, FOF, LE).

Epiphyte. Primary and secondary broad-leaved evergreen dry forests on any kind of soil, preferably on rocky limestone, 1000-1450 m. Fl. August September. Not common.

NE. India, S. China, Thailand, Vietnam. Laos: Champasak, Khammouan, Louangphrabang, Saravan, Vientiane, Xiangkhouang.

\section{D. porphyrochilum Lindl.,}

1858, Journ. Proc. Linn. Soc., Bot. 3: 18; Schuit. et al., 2008, Nord. Journ. Bot. 26: 286.

Central Laos, Xiangkhouang province, Peak district, Oran village, 2 April 2015, N. T. Hiep, L. Averyanov, N. S. Khang et al., LA-VN 921 / TM 1229 (HNL, FOF, LE).

Epiphyte on tall trees. Primary and secondary broad-leaved evergreen dry forests on shale sandstone along ridge edges, 1750-1850 m. Fl. July. Rare.

India, Nepal, Bhutan, Myanmar, China, Thailand. Laos: Champasak, Xiangkhouang.

D. salaccense Lindl.,

1830, Gen. Sp. Orch. Pl.: 86; Newman et al., 2007, Checklist Vasc. Pl. Lao PDR: 265; Schuit. et al., 2008, Nord. Journ. Bot. 26: 286.

Northeastern Laos, Houaphan province, Hem district, Na Puok village, 11 April 2015, N. T. Hiep,
L. Averyanov, N. S. Khang et al., LA-VN 1294 (HNL, FOF, LE).

Epiphyte and lithophyte. Primary and secondary broad-leaved evergreen and deciduous dry dipterocarp and limestone forests, often in humid shady wet places, 300-1150 m. Fl. April - October. Occasional.

Myanmar, Thailand, Vietnam, Malacca Peninsula, Sumatra, Java, Borneo. Laos: Bolikhamxai, Houaphan, Khammouan.

D. senile Rchb. f.,

1865, Gard. Chron. 1865: 434; Newman et al., 2007, Checklist Vasc. P1. Lao PDR: 265; Schuit. et al., 2008, Nord. Journ. Bot. 26: 287; Aver., 2012, Taiwania 57, 2: 131.

Fig. 7A.

Central Laos, Vientiane province, Phou Khao Khouay National Protected Area to about $40 \mathrm{~km}$ NE of Vientiane, 9 December 2015, L. Averyanov, T. Maisak, AL 158a (LE - photo). Southwestern Laos, Champasak province, Pakse district, Bolaven plateau, along road Paksong - Ban Houay Kong, 26 November 2015, E. Konstantinov, T. Vongsa, K-400 (LE - photo).

Epiphyte and lithophyte. Primary and secondary evergreen broad-leaved and coniferous dry open forests and woodlands, commonly on sandstone along streams and rivers, 200-1500 m. Fl. January April. Rare. Ornamental.

Burma, Thailand, Vietnam. Laos: Bolikhamxai, Champasak, Vientiane, Xiangkhouang.

A great amount of this species specimens observed in Vietnamese street markets in 2014-2016 was originated certainly from northern Laos where it may be still very common. In Vietnam this species is extremely rare and stands on the verge of full extinction (Averyanov, 2012).

D. thyrsiflorum B. S. Williams,

1871, Orch.-Grow. Man. ed. 4: 172; Newman et al., 2007, Checklist Vasc. Pl. Lao PDR: 265; Schuit. et al., 2008, Nord. Journ. Bot. 26: 288.

Central Laos, Xiangkhouang province, Kham district, Huad village, 4 April 2015, N. T. Hiep, L. Averyanov, N. S. Khang et al., LA-VN 1058 (HNL, FOF, LE). Central Laos, Xiangkhouang province, Peak district, Khoang village, 3 April 2015, N. T. Hiep, L. Averyanov, N. S. Khang et al., LA-VN 970 (HNL, FOF, LE). Northeastern Laos, Houaphan province, Xon district, Nong Hua Pa Noi village, 11 April 2015, N. T. Hiep, L. Averyanov, N. S. Khang et al., LA-VN 1237 (HNL, FOF, LE). 


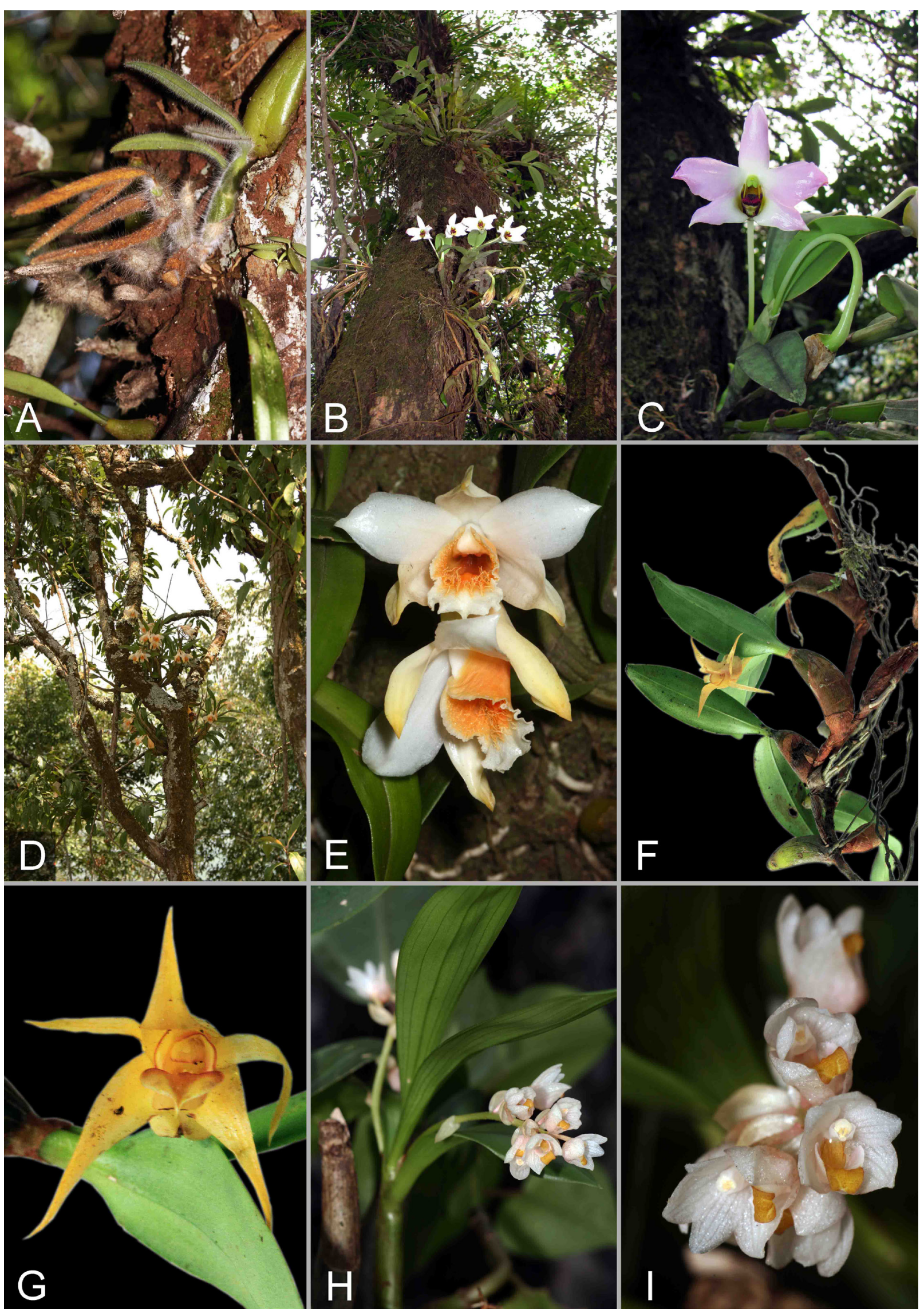

Fig. 7. New and rare orchids in the flora of Cambodia and Laos. A - Dendrobium senile Rchb.f. $(A L 158 a)$. B, C D. trantuanii Perner et X.N. Dang (LA-VN 1402). D, E - D. williamsonii Day et Rchb.f. (CPC 2429). F, G - Epigeneium fuscescens (Griff.) Summerh. (K-98). H, I - Eria apertiflora Summerh. (LA-VN 974 / TM 1247). Photos by L. Averyanov (A, D, E, H, I), K. S. Nguyen (B, C) and E. Konstantinov (F, G). 
Epiphyte. Primary and secondary broad-leaved and mixed evergreen and deciduous dry forests on any kind of soil, commonly along stream valleys and on mountain tops, 700-1450 m. Fl. March May. One of the most frequent epiphytic orchids in Laos. Ornamental.

NE. India, Myanmar, S. China, Thailand, Vietnam. Laos: Champasak, Houaphan, Khammouan, Louang Namtha, Louangphrabang, Phongsali, Vientiane, Xiangkhouang.

Almost all collections from Laos belong to smallflowered variety $-D$. thyrsiflorum var. minutiflorum Aver., 2012, Taiwania 57, 2: 131.

${ }^{\mathrm{L}}$ D. trantuanii Perner et X. N. Dang,

2003, Orchidee (Hamburg) 54: 220.

$=D$. tuananhii Aver., 2004, Orchids (West Palm Beach) 73: 134.

Fig. 7B, C.

Northeastern Laos, Houaphan province, Hem district, Nacoc village, 12 April 2015, N. T. Hiep, L. Averyanov, N. S. Khang et al., LA-VN 1402 (HNL, FOF, LE).

Epiphyte. Primary and secondary broad-leaved evergreen open dry forests on rocky limestone, 1000-1200 m. Fl. March - May. Very rare. Ornamental.

NW. Vietnam. Laos: Houaphan.

D. trigonopus Rchb. f.,

1887, Gard. Chron. 1887, 2: 682; Newman et al., 2007, Checklist Vasc. Pl. Lao PDR: 265; Schuit. et al., 2008, Nord. Journ. Bot. 26: 288.

Central Laos, Xiangkhouang province, Kham district, Huad village, 4 April 2015, N. T. Hiep, L. Averyanov, N. S. Khang et al., LA-VN 1095 (HNL, FOF, LE).

Epiphyte. Primary and secondary broad-leaved evergreen and semideciduous dry forests on rocky limestone, commonly on mountain tops, $1200-1800$ m. Fl. March - May. Not common. Ornamental.

Myanmar, China, Thailand, Vietnam. Laos: Khammouan, Vientiane, Xiangkhouang.

${ }^{\mathrm{L}}$ D. williamsonii Day et Rchb. f., 1869, Gard. Chron. 1869: 78.

$=D$. cariniferum auct. non Rchb. f.: Aver., 2013, Turczaninowia 16, 4: 34.

Fig. 7D, E.

Northern Laos, Phongsali province, Muong May district, 22 April 2011, L. Averyanov, P.V. The, CPC 2429 (LE - photo). Northeastern Laos, Houaphan province, Xon district, Nong Hua Pa Noi village, 11
April 2015, N. T. Hiep, L. Averyanov, N. S. Khang et al., LA-VN 1227, 1290a (HNL, FOF, LE).

Epiphyte. Primary and secondary broad-leaved evergreen forests on any kind of soil, commonly on mountain tops and along ridge edges, $1100-1400 \mathrm{~m}$. Fl. March - May. Locally common. Ornamental.

NE. India, Myanmar, S. China, Vietnam. Laos: Phongsali, Houaphan.

${ }^{\mathrm{L}}$ Didymoplexis Griff.

${ }^{\mathrm{L}} D$. pallens Griff.,

1844, Calcutta Journal Nat. Hist., 4: 383; id., 1845, ibid., 5, pl. 17.

Central Laos, Xiangkhouang province, Peak district, Oran village, 2 April 2015, N. T. Hiep, L. Averyanov, N. S. Khang et al., LA-VN 930 (HNL, FOF, LE).

Miniature terrestrial achlorophyllous mycotrophic herb. Primary and secondary broad-leaved evergreen dry forests on shale sandstone along ridge edges on rich soil, 1750-1850 m. Fl. May - June. Very rare.

Tropical and subtropical mainland Asia, Ryukyu Islands, Malay Archipelago to New Guinea. Laos: Xiangkhouang.

Doritis Lindl.

D. pulcherrima Lindl.,

1833, Gen. Sp. Orch. P1.: 178; Aver., 2013, Turczaninowia 16, 4: 36.

$\equiv$ Phalaenopsis pulcherrima (Lindl.) J. J. Sm., 1933, Repert. Spec. Nov. Regni Veg. 32: 366; Newman et al., 2007, Checklist Vasc. Pl. Lao PDR: 277; Schuit. et al., 2008, Nord. Journ. Bot. 26: 305.

Central Laos, Vientiane province, Phou Khao Khouay National Protected Area to about $40 \mathrm{~km}$ NE of Vientiane, 9 December 2015, L. Averyanov, T. Maisak, AL 159c (LE - photo).

Lithophyte on exposed rocks commonly along streams, rarely terrestrial or epiphyte in dry open semideciduous and deciduous forests, 300-1300 m. Fl. June - September. Not rare. Ornamental.

NE. India, Myanmar, S. China, Thailand, Cambodia, Vietnam, Malacca Peninsula, Sumatra, Borneo. Laos: Bolikhamxai, Champasak, Khammouan, Savannakhet, Vientiane.

Epigeneium Gagnep.

E. amplum (Wall.) Summerh.,

1957, Kew Bull. 12: 260; Schuit. et al., 2008, Nord. Journ. Bot. 26: 289.

Central Laos, Xiangkhouang province, Peak district, Khoang village, 3 April 2015, N. T. Hiep, 
L. Averyanov, N. S. Khang et al., LA-VN 986 (HNL, FOF, LE).

Creeping epiphyte and occasionally lithophyte. Primary and secondary broad-leaved evergreen forests on any kind of soil, preferably on rocky limestone, commonly on mountain tops, $1200-1450$ m. Fl. October - December. Occasional.

NE. India, Nepal, Bhutan, Myanmar, China, Thailand, Vietnam. Laos: Louang Namtha, Xiangkhouang.

\section{${ }^{\mathrm{L}}$ E. fuscescens (Griff.) Summerh.,}

1957, Kew Bull. 12: 262.

Fig. 7F, G.

Central Laos, Vientiane province, Kasi district, Namken village, 24 March 2013, L. Averyanov, N. S. Khang, S. Lorphengsy, LA-VN 798 (HNL, FOF, LE). Central Laos, Vientiane province, Kasi district, Thong Mout village, pass on new road to Louangphrabang, 21 October 2013, E. Konstantinov et al., $K-98$ (LE).

Creeping epiphyte and occasionally lithophyte. Primary broad-leaved evergreen forests on rocky limestone, commonly on mountain tops, $1500-1800$ m. Fl. September - November. Locally common.

NE. India, Bhutan, Nepal, S. China. Laos: Vientiane.

\section{Eria Lindl.}

\section{E. acervata Lindl.,}

1851, Journ. Hort. Soc. London 6: 57; Newman et al., 2007, Checklist Vasc. Pl. Lao PDR: 267; Schuit. et al., 2008, Nord. Journ. Bot. 26: 289.

Northeastern Laos, Houaphan province, Hem district, Na Puok village, 11 April 2015, N. T. Hiep, L. Averyanov, N. S. Khang et al., LA-VN 1307 (HNL, FOF, LE).

Epiphyte. Primary and secondary broad-leaved evergreen dry forests on rocky limestone, commonly on mountain tops, 1050-1150 m. Fl. May - June. Not common.

NE. India, Nepal, Bhutan, Myanmar, China, Thailand, Cambodia, Vietnam. Laos: Champasak, Houaphan, Louangphrabang.

\section{${ }^{\mathrm{L}}$ E. apertiflora Summerh.,}

1929, Bull. Misc. Inform. Kew 1929: 9.

Fig. 7H, I.

Central Laos, Xiangkhouang province, Kham district, Huad village, 4 April 2015, N. T. Hiep, $L$. Averyanov, N. S. Khang et al., LA-VN 1093a / TM 1262 (HNL, FOF, LE). Central Laos, Xiangkhouang province, Peak district, Khoang village, 3 April
2015, N. T. Hiep, L. Averyanov, N. S. Khang et al., LA-VN 974 / TM 1247 (HNL, FOF, LE).

Epiphyte on tall trees. Primary and secondary broad-leaved evergreen and semideciduous dry forests on rocky limestone, $1200-1450 \mathrm{~m}$. Fl. August - October. Locally very common.

NE. India, Nepal, Myanmar, Thailand, Vietnam. Laos: Xiangkhouang.

${ }^{\mathrm{L}}$ E. bambusifolia Lindl.,

1858, Journ. Proc. Linn. Soc., Bot. 3: 61.

Northeastern Laos, Houaphan province, Viengxay district, Xieng Luong village, 8 April 2015, $N$. T. Hiep, L. Averyanov, N. S. Khang et al., LA-VN 1162 (HNL, FOF, LE).

Lithophyte and occasionally epiphyte. Primary and secondary broad-leaved evergreen dry forests on rocky limestone, commonly on shady mossy rocks on mountain tops, $600-700 \mathrm{~m}$. Fl. SeptemberNovember. Rare.

India, Bhutan, Myanmar, SW. China (Yunnan), Thailand, Vietnam. Laos: Houaphan.

\section{E. lasiopetala (Willd.) Ormerod,}

1995, Opera Bot. 124: 22; Newman et al., 2007, Checklist Vasc. P1. Lao PDR: 268; Schuit. et al., 2008, Nord. Journ. Bot. 26: 290; Aver., 2013, Turczaninowia 16, 4: 37 .

$=$ E. albidotomentosa (Blume) Lindl., 1830, Gen. Sp. Orch. Pl.: 66.

=E. pubescens (Hook.) Lindl., 1830, Hort. Brit.: 372.

Fig. 8A, B.

Central Laos, Vientiane province, Phou Khao Khouay National Protected Area to about $40 \mathrm{~km}$ NE of Vientiane, 9 December 2015, L. Averyanov, T. Maisak, AL $159 b$ (LE - photo). Northeastern Laos, Houaphan province, Hem district, Nacoc village, 12 April 2015, N. T. Hiep, L. Averyanov, N. S. Khang et al., LA-VN 1461 (HNL, FOF, LE).

Creping epiphyte and lithophyte. Primary and secondary broad-leaved evergreen and deciduous open dry forests on any kind of soil, often on mountain tops, in gallery forests and on exposed mossy stream rocks, 300-1200 m. Fl. February April, September - December. Not rare.

NE. India, Nepal, Bhutan, Myanmar, S. China, Thailand, Vietnam, Cambodia, Malacca Peninsula, Sumatra, Java. Laos: Bolikhamxai, Champasak, Houaphan, Khammouan, Saravan, Vientiane.

E. muscicola (Lindl.) Lindl.,

1858, Journ. Proc. Linn. Soc., Bot. 3: 47; Newman et al., 2007, Checklist Vasc. Pl. Lao PDR: 


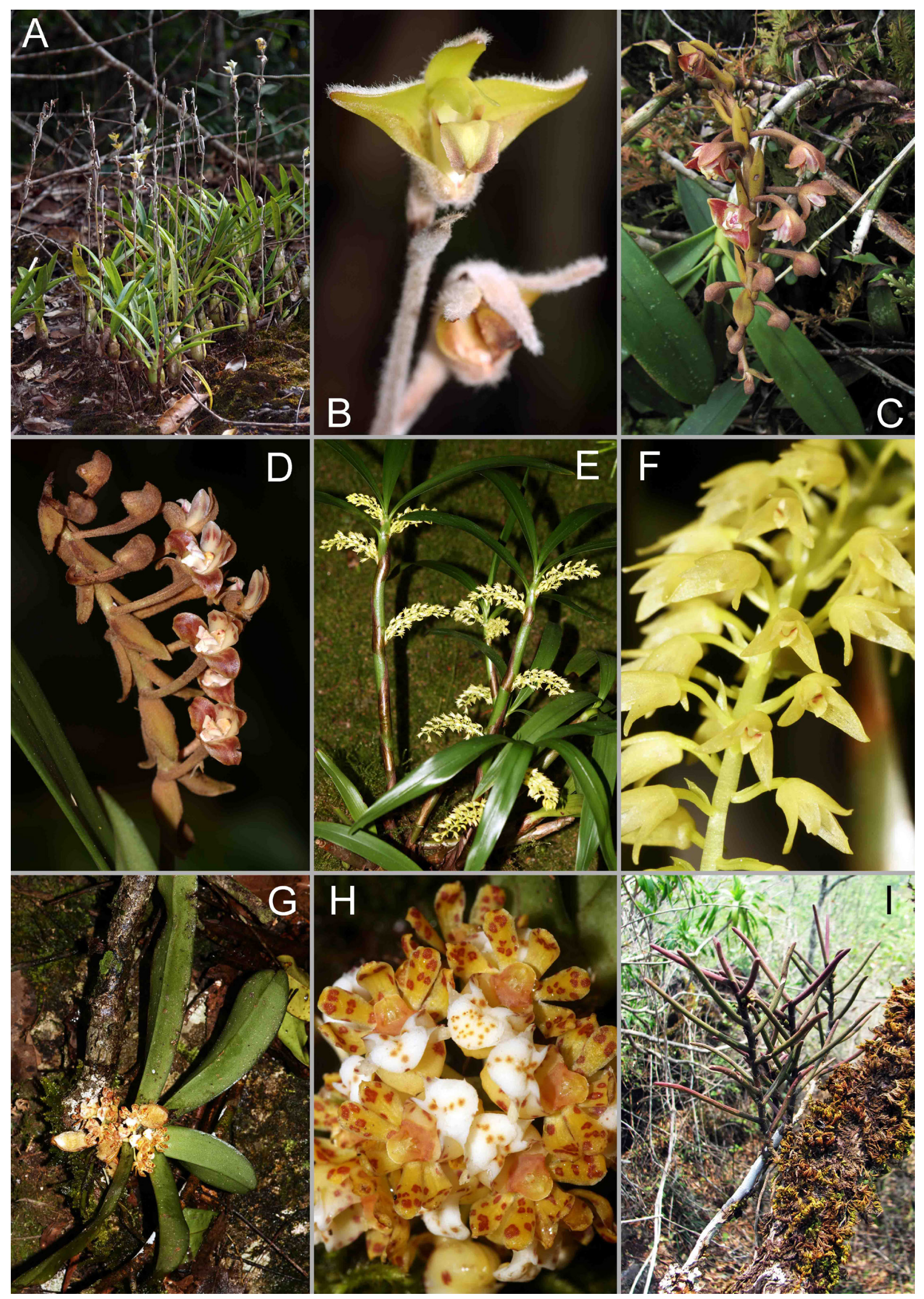

Fig. 8. New and rare orchids in the flora of Cambodia and Laos. A, B - Eria lasiopetala (Willd.) Ormerod ( $A L$ 159b). C, D - E. pachyphylla Aver. (LA-VN 1208). E. tenuiflora Ridl. (T. Maisak et al., 931). G, H - Gastrochilus suavis Seidenf. (LA-VN 1131). I - Luisia ramosii Ames (LA-VN 1098). Photos by L. Averyanov (A, B, D-H) and K. S. Nguyen $(\mathrm{C}, \mathrm{I})$. 
268; Schuit. et al., 2008, Nord. Journ. Bot. 26: 291; Aver., 2013, Turczaninowia 16, 4: 37.

Northeastern Laos, Houaphan province, Xon district, Nong Hua Pa Noi village, 11 April 2015, N. T. Hiep, L. Averyanov, N. S. Khang et al., LA-VN 1268 (HNL, FOF, LE).

Epiphyte on tall trees. Primary and secondary broad-leaved evergreen forests on shaly limestone, 1100-1500 m. Fl. August. Rare.

India, Nepal, Bhutan, Myanmar, SW. China (Yunnan), Thailand, Cambodia, Vietnam. Laos: Houaphan, Vientiane.

${ }^{\mathrm{L}}$ E. pachyphylla Aver.,

2002, Turczaninowia 5, 4: 77.

$=$ E. crassifolia Z. H. Tsi et S. C. Chen, 1994, Acta Phytotax. Sin. 32: 561, not Ridley, 1915.

Fig. 8C, D.

Northeastern Laos, Houaphan province, Viengxay district, Xieng Luong village, 8 April 2015, N. T. Hiep, L. Averyanov, N. S. Khang et al., $L A-V N 1208$ (HNL, FOF, LE).

Lithophyte and occasionally epiphyte. Primary and secondary broad-leaved evergreen dry open forests on rocky limestone, commonly on mossy hill and mountain tops, 600-700 m. Fl. March - May. Not common.

S. China, N. Vietnam. Laos: Houaphan.

\section{E. paniculata Lindl.,}

1830, Pl. Asiat. Rar. 1: 32; Newman et al., 2007, Checklist Vasc. Pl. Lao PDR: 268; Schuit. et al., 2008, Nord. Journ. Bot. 26: 291; Aver., 2013, Turczaninowia 16, 4: 37.

Central Laos, Xiangkhouang province, Peak district, Khoang village, 3 April 2015, N. T. Hiep, L. Averyanov, N. S. Khang et al., LA-VN 982 (HNL, FOF, LE). Northeastern Laos, Houaphan province, Xon district, Nong Hua Pa Noi village, 11 April 2015, N. T. Hiep, L. Averyanov, N. S. Khang et al., $L A-V N 1283$ (HNL, FOF, LE).

Epiphyte. Primary and secondary broad-leaved evergreen forests on any kind of soil, commonly on mountain tops, solid rocky highly eroded limestone, 1000-1750 m. Fl. March - June. Not rare.

NE. India, Nepal, Bhutan, Bangladesh, Myanmar, Thailand, Cambodia, Vietnam. Laos: Attapu, Champasak, Houaphan, Saravan, Vientiane, Xiangkhouang.

\section{E. pannea Lindl.,}

1842, Bot. Reg. 28 Misc.: 64; Newman et al., 2007, Checklist Vasc. P1. Lao PDR: 268; Schuit. et al., 2008, Nord. Journ. Bot. 26: 291; Aver., 2013, Turczaninowia 16, 4: 37.

Central Laos, Xiangkhouang province, Kham district, Huad village, 4 April 2015, N. T. Hiep, L. Averyanov, N. S. Khang et al., LA-VN 1093 (HNL, FOF, LE). Central Laos, Xiangkhouang province, Peak district, Khoang village, 3 April 2015, N. T. Hiep, L. Averyanov, N. S. Khang et al., LA-VN 989 (HNL, FOF, LE). Northeastern Laos, Houaphan province, Hem district, Na Puok village, 11 April 2015, N. T. Hiep, L. Averyanov, N. S. Khang et al., LA-VN 1330 (HNL, FOF, LE). Northeastern Laos, Houaphan province, Hem district, Nacoc village, 12 April 2015, N. T. Hiep, L. Averyanov, N. S. Khang et al., LA-VN 1458 (HNL, FOF, LE). Northeastern Laos, Houaphan province, Xon district, Nong Hua Pa Noi village, 11 April 2015, N. T. Hiep, L. Averyanov, N. S. Khang et al., LA-VN 1238 (HNL, FOF, LE).

Creeping epiphyte. Primary and secondary broad-leaved and coniferous evergreen and deciduous open forests and woodlands on any kind of soil, commonly on tall trees and on mountain tops, 1501500 m. Fl. March - June (July). One of the commonest epiphytic orchid in Laos.

NE. India, Myanmar, S. China, Thailand, Cambodia, Vietnam, Malacca Peninsula, Sumatra, Borneo. Laos: Bolikhamxai, Champasak, Houaphan, Khammouan, Louang Namtha, Phongsali, Saravan, Vientiane, Xiangkhouang.

${ }^{\mathrm{C}}$ E. tenuiflora Ridl.,

1896, Journal. Linn. Soc., Bot. 32: 291.

Fig. 8E, F.

Southern Cambodia, Kampot province, Bokor National Park, Bokor mt., 24 November 2012, T. Maisak, M. Telepova, L. Osinovetz, I.Kutuzova, 931 (LE). Southern Cambodia, Kampot province, Bokor National park, 1 December 2014, E. Konstantinov, $K-364$ (LE). Southern Cambodia, to the N of Sihanoukville town, Kaoh Rong Island in Siam Gulf, 28 November 2012, T. Maisak, M. Telepova, L. Osinovetz, I. Kutuzova, 1012 (LE).

Epiphyte. Primary and secondary evergreen broad-leaved dry lowland forest on low hills to humid cloud forests on mountain tops, 50-1050 m. Fl. November - January. Not common.

Thailand, Vietnam, Malacca Peninsula, Sumatra, Java, Kalimantan. Cambodia: Kampot, Sihanoukville.

E. tomentosa Hook. f.,

1890, Fl. Brit. India 5: 803; Newman et al., 2007, Checklist Vasc. P1. Lao PDR: 268; Schuit. et al., 
2008, Nord. Journ. Bot. 26: 292.

Northeastern Laos, Houaphan province, Xon district, Nong Hua Pa Noi village, 11 April 2015, N. T. Hiep, L. Averyanov, N. S. Khang et al., LA-VN 1234 (HNL, FOF, LE).

Epiphyte and occasionally lithophyte. Primary and secondary broad-leaved evergreen forests on any kind of soil, 150-1200 m. Fl. May - June. Not common.

NE. India, Myanmar, Thailand, Vietnam. Laos: Champasak, Houaphan, Louangphrabang, Phongsali, Xaignabouri, Xiangkhouang.

Flickingeria A. D. Hawkes

F. angustifolia (Blume) A. D. Hawkes,

1965, Orquidea (Rio de Janeiro) 27: 301; Aver., 2013, Turczaninowia 16, 4: 37.

Northeastern Laos, Houaphan province, Viengxay district, Vieng Xai village, 7 April 2015, N. T. Hiep, L. Averyanov, N. S. Khang et al., LA$V N 1133$ (HNL, FOF, LE). Northeastern Laos, Houaphan province, Viengxay district, Xieng Luong village, 8 April 2015, N. T. Hiep, L. Averyanov, N. S. Khang et al., LA-VN 1187 (HNL, FOF, LE).

Epiphyte. Primary and secondary broad-leaved evergreen dry forests on any kind of soil, preferably on rocky limestone, 500-1350 m. Fl. June September. Locally very common.

S. China, Thailand, Vietnam, Malacca Peninsula, Indonesia. Laos: Houaphan, Saravan, Vientiane.

${ }^{\mathrm{L}} \boldsymbol{F}$. fimbriata (Blume) A. D. Hawkes, 1965, Orquidea (Rio de Janeiro) 27: 303.

Central Laos, Xiangkhouang province, Peak district, Khoang village, 3 April 2015, N. T. Hiep, L. Averyanov, N. S. Khang et al., LA-VN 997 (HNL, FOF, LE). Northeastern Laos, Houaphan province, Hem district, Na Puok village 11 April 2015, N. T. Hiep, L. Averyanov, N. S. Khang et al., LA-VN 1297 (HNL, FOF, LE). Northeastern Laos, Houaphan province, Hem district, Na Puok village, 11 April 2015, N. T. Hiep, L. Averyanov, N. S. Khang et al., $L A-V N 1326$ (HNL, FOF, LE).

Epiphyte and lithophyte. Primary and secondary broad-leaved evergreen dry forests on rocky limestone, commonly on mossy mountain tops, 1000-1450 m. Fl. April - June. Locally common.

Nicobar and Andaman Islands, S. China, Thailand, Vietnam, Malacca Peninsula, Indonesia, Philippines. Laos: Houaphan, Xiangkhouang.

Gastrochilus D. Don.

${ }^{\mathrm{L}}$ G. suavis Seidenf.,

1988, Opera Bot. 95: 298.
$=$ G. obliquus (Lindl.) Kuntze var. suavis (Seidenf.) Z. H. Tsi, 1996, Guihaia 16: 141.

Fig. $8 \mathrm{G}, \mathrm{H}$.

Northeastern Laos, Houaphan province, Hem district, Na Puok village, 11 April 2015, N. T. Hiep, L. Averyanov, N. S. Khang et al., LA-VN 1301 (HNL, FOF, LE). Northeastern Laos, Houaphan province, Viengxay district, Vieng Xai village, 7 April 2015, N. T. Hiep, L. Averyanov, N. S. Khang et al., LA-VN 1131 (HNL, FOF, LE).

Epiphyte. Primary and secondary broad-leaved evergreen dry forests on rocky limestone, commonly on shady humid places on hill and mountain tops, 800-1200 m. Fl. March - May. Not common. Ornamental.

Thailand, Vietnam. Laos: Houaphan.

This speces regarded earlier as endemic of Thailand (Seidenfaden, 1988) was recently also found in northern Vietnam: Quang Binh province, Minh Hoa district, Thuong Hoa municipality, environs of Mo O O O village, L. Averyanov et al., CPC 3661 (LE); Thanh Hoa province, Thuong Xuan district, Van Xuan Municipality, Xuan Lien Natural Reserve, $L$. Averyanov et al., CPC 6932 (LE).

Goodyera R. Br.

G. procera Hook.,

1823, Exot. Fl. 1, t. 39; Newman et al., 2007, Checklist Vasc. P1. Lao PDR: 270; Schuit. et al., 2008, Nord. Journ. Bot. 26: 293.

Northeastern Laos, Houaphan province, Hem district, Nacoc village, 12 April 2015, N. T. Hiep, $L$. Averyanov, N. S. Khang et al., LA-VN 1390 (HNL, FOF, LE).

Terrestrial and occasionally lithophytic herb in open wet mossy places on any kind of soil and in any plant communities, commonly along sandy banks of streams, wet rocks and rocky waterfalls, 400-900 m. Fl. March - May. Not rare.

Sri Lanka, India, Nepal, Bhutan, Myanmar, China, Taiwan, Japan, Thailand, Cambodia, Vietnam, Malacca Peninsula, Sumatra, Java, Borneo, Lesser Sunda Islands, Philippines. Laos: Attapu, Houaphan, Louangphrabang, Xiangkhouang.

\section{Hetaeria Blume}

H. affinis (Griff.) Seidenf. et Ormerod,

2001, Oasis Suppl. 2: 9;. Aver., 2013, Turczaninowia 16, 4: 38 .

$=$ Goodyera affinis Griff., 1851, Not. Pl. Asiat. 3: 391 .

= Hetaeria rubens (Lindl.) Hook. f., 1890, Fl. Brit. India 6: 115. 
Northeastern Laos, Houaphan province, Viengxay district, Xieng Luong village, 8 April 2015, $N$. T. Hiep, L. Averyanov, N. S. Khang et al., LA-VN 1170 (HNL, FOF, LE).

Terrestrial herb. Primary and secondary broadleaved evergreen dry forests on rocky limestone, usually in shady humid depressions on rich soil, 300-700 m. Fl. March - May. Not common.

NE. India, Nepal, Bhutan, Myanmar, S. China, Thailand, Vietnam. Laos: Houaphan, Vientiane.

\section{Holcoglossum Schltr.}

H. kimballianum (Rchb. f.) Garay,

1972, Bot. Mus. Leafl. 23: 182; Newman et al., 2007, Checklist Vasc. P1. Lao PDR: 272; Schuit. et al., 2008, Nord. Journ. Bot. 26: 295.

$=H$. saprophyticum (Gagnep.) Christenson, 1987, Notes Roy. Bot. Gard. Edinburgh 44: 255.

Central Laos, Vientiane province, Kasi district, Thong Mout village, 20 March 2013, N. T. Hiep, L. Averyanov, N. S. Khang et al., LA-VN $606 a$ (LE).

Epiphyte. Primary broad-leaved evergreen forests on rocky limestone, $1550-1650 \mathrm{~m}$. Fl. September - October. Rare. Ornamental.

Myanmar, SW. China (Yunnan), Thailand, Vietnam. Laos: Vientiane, Xiangkhouang.

\section{H. subulifolium (Rchb. f.) Christenson,}

1987, Notes Roy. Bot. Gard. Edinburgh 44: 255; Aver., 2013, Turczaninowia 16, 4: 38.

= Vanda watsonii Rolfe, 1905, Gard. Chron. 1: 82.

Central Laos, Xiangkhouang province, Peak district, Khoang village, 3 April 2015, N. T. Hiep, L. Averyanov, N. S. Khang et al., LA-VN 983 (HNL, FOF, LE).

Epiphyte. Primary and secondary broad-leaved evergreen dry forests on rocky limestone, commonly on mountain tops, $1200-1450 \mathrm{~m}$. Fl. March April. Rare. Ornamental.

Myanmar, S. China, Thailand, Vietnam. Laos: Khammouan, Xiangkhouang.

\section{Hygrochilus Pfitzer \\ H. parishii Pfitzer,}

1897, Nat. Pflanzenfam. Nachtr. 1: 112; Newman et al., 2007, Checklist Vasc. P1. Lao PDR: 272; Schuit. et al., 2008, Nord. Journ. Bot. 26: 295.

Central Laos, Xiangkhouang province, Peak district, Khoang village, 3 April 2015, N. T. Hiep, L. Averyanov, N. S. Khang et al., LA-VN 992 (HNL, FOF, LE). Northeastern Laos, Houaphan province, Viengxay district, Xieng Luong village, 8 April
2015, N. T. Hiep, L. Averyanov, N. S. Khang et al., LA-VN 1194 (HNL, FOF, LE).

Epiphyte and lithophyte. Primary and secondary broad-leaved evergreen dry open forests preferably on rocky limestone, commonly on mountain tops, 600-1450 m. Fl. April - June. Occasional. Ornamental.

NE. India, Myanmar, Thailand, Vietnam. Laos: Champasak, Houaphan, Phongsali, Xiangkhouang.

\section{Liparis Rich.}

L. bootanensis Griff.,

1851, Not. Pl. Asiat. 3: 278; Newman et al., 2007, Checklist Vasc. Pl. Lao PDR: 272; Schuit. et al., 2008, Nord. Journ. Bot. 26: 295; Aver., 2013, Turczaninowia 16, 4: 38.

Central Laos, Xiangkhouang province, Peak district, Oran village, 2 April 2015, N. T. Hiep, L. Averyanov, N. S. Khang et al., LA-VN 921a / TM 1228, 1235 (LE).

Epiphyte and occasionally lithophyte. Primary and secondary broad-leaved evergreen dry forests on any kind of soil, commonly on mountain tops, 1200-1850 m. Fl. March - July, September October. Occasional.

NE. India, Bhutan, S. China, Taiwan, Myanmar, Japan, Thailand, Vietnam, Malacca Peninsula, Java, Kalimantan, Lesser Sunda Islands, Philippines. Laos: Khammouan, Phongsali, Xiangkhouang.

\section{L. cespitosa (Lam.) Lindl.,}

1825, Bot. Reg. 11, t. 882; Newman et al., 2007, Checklist Vasc. P1. Lao PDR: 272; Schuit. et al., 2008, Nord. Journ. Bot. 26: 295. 187.

E Epidendrum cespitosa Lam., 1783, Encycl. 1:

= Malaxis caespitosa Thouars, 1822, Hist. Orchid., t. 90.

Central Laos, Xiangkhouang province, Peak district, Oran village, 2 April 2015, N. T. Hiep, L. Averyanov, N. S. Khang et al., LA-VN 921 / TM 1213 (HNL, FOF, LE).

Miniature clustering epiphyte. Primary and secondary broad-leaved evergreen montane forests on any kind of soil, 800-1850 m. Fl. August - October. Not rare.

Tropical area of the Old World extending to islands of western Pacific. Laos: Bolikhamxai, Champasak, Phongsali, Vientiane, Xiangkhouang.

\section{${ }^{\mathrm{L}}$ L. mannii Rchb. f.,}

1872, Flora 55: 275.

Northeastern Laos, Houaphan province, Hem district, Khon Ngua village, 15 April 2015, N. T. 
Hiep, L. Averyanov, N. S. Khang et al., LA-VN 1464 (HNL, FOF, LE). Northeastern Laos, Houaphan province, Viengxay district, Vieng Xai village 7 April 2015, N. T. Hiep, L. Averyanov, N. S. Khang et al., LA-VN 1141 (HNL, FOF, LE). Northeastern Laos, Houaphan province, Viengxay district, Xieng Luong village, 8 April 2015, N. T. Hiep, L. Averyanov, N. S. Khang et al., LA-VN 1196 (HNL, FOF, LE).

Epiphyte and lithophyte. Primary and secondary broad-leaved evergreen forests on rocky limestone, commonly on hill and mountain tops, 600-950 m. Fl. October - April. Locally common.

NE. India, Bhutan, S. China, Vietnam. Laos: Houaphan.

\section{L. pumila Aver.,}

2003, Updated Checklist Orchids Vietnam: 42, 85, fig. 7; Aver., 2013, Turczaninowia 16, 4: 38.

Central Laos, Xiangkhouang province, Kham district, Huad village, 4 April 2015, N. T. Hiep, L. Averyanov, N. S. Khang et al., LA-VN 1050 (HNL, FOF, LE). Central Laos, Xiangkhouang province, Peak district, Khoang village, 3 April 2015, N. T. Hiep, L. Averyanov, N. S. Khang et al., LA-VN 976 (HNL, FOF, LE). Northeastern Laos, Houaphan province, Hem district, Na Puok village, 11 April 2015, N. T. Hiep, L. Averyanov, N. S. Khang et al., $L A-V N 1318$ (HNL, FOF, LE). Northeastern Laos, Houaphan province, Hem district, Nacoc village, 12 April 2015, N. T. Hiep, L. Averyanov, N. S. Khang et al., LA-VN 1396 (HNL, FOF, LE).

Miniature clustering epiphyte. Primary and secondary broad-leaved evergreen and semideciduous forests on rocky limestone, 500-1450 m. Fl. February - April. Not rare.

S. China, Vietnam. Laos: Bolikhamxai, Houaphan, Vientiane, Xiangkhouang.

${ }^{\mathrm{L}}$ L. sootenzanensis Fukuy., 1933, Ann. Rep. Taihoku Bot. Gard. 3: 84.

Northeastern Laos, Houaphan province, Hem district, Khon Ngua village, 15 April 2015, N. T. Hiep, L. Averyanov, N. S. Khang et al., LA-VN 1499 (HNL, FOF, LE).

Terrestrial herb. Primary and secondary broadleaved evergreen forests, sometime secondary bamboo thickets on rocky limestone, commonly in shady depressions on rich soil, 700-950 m. Fl. April May. Rare.

Taiwan, Vietnam. Laos: Houaphan.

\section{L. viridiflora Lindl.,}

1830, Gen. Sp. Orch. Pl.: 31; Newman et al., 2007, Checklist Vasc. Pl. Lao PDR: 273; Schuit. et al., 2008, Nord. Journ. Bot. 26: 296; Aver., 2013, Turczaninowia 16, 4: 38.

Central Laos, Xiangkhouang province, Kham district, Huad village, 4 April 2015, N. T. Hiep, L. Averyanov, N. S. Khang et al., LA-VN 1065 (HNL, FOF, LE). Northeastern Laos, Houaphan province, Hem district, Khon Ngua village, 15 April 2015, N. T. Hiep, L. Averyanov, N. S. Khang et al., LA-VN 1492 (HNL, FOF, LE). Northeastern Laos, Houaphan province, Hem district, Nacoc village, 12 April 2015, N. T. Hiep, L. Averyanov, N. S. Khang et al., LA-VN 1442 (HNL, FOF, LE). Northeastern Laos, Houaphan province, Viengxay district, Vieng Xai village, 7 April 2015, N. T. Hiep, L. Averyanov, N. S. Khang et al., LA-VN 1151 (HNL, FOF, LE). Northeastern Laos, Houaphan province, Viengxay district, Xieng Luong village, 8 April 2015, N. T. Hiep, L. Averyanov, N. S. Khang et al., LA-VN 1181 (HNL, FOF, LE).

Epiphyte and lithophyte. Primary and secondary evergreen and semideciduous forests, secondary shrubs on any kind of rocks, but more common on rocky limestone, $500-1650 \mathrm{~m}$. Fl. October January (April). Very common.

Tropical Asia, tropical islands of western Pacific. Laos: Bolikhamxai, Champasak, Houaphan, Khammouan, Louang Namtha, Louangphrabang, Saravan, Vientiane, Xiangkhouang.

\section{Luisia Gaudich.}

${ }^{\mathrm{L}}$ L. ramosii Ames,

1911, Philipp. Journ. Sci., C 6: 55.

Fig. 8I; 10A.

Central Laos, Xiangkhouang province, Kham district, Huad village, 4 April 2015, N. T. Hiep, L. Averyanov, N. S. Khang et al., LA-VN 1036 (HNL, FOF, LE). Central Laos, Xiangkhouang province, Peak district, Khoang village, 3 April 2015, N. T. Hiep, L. Averyanov, N. S. Khang et al., LA-VN 1098 (HNL, FOF, LE). Northeastern Laos, Houaphan province, Hem district, Khon Ngua village, 15 April 2015, N. T. Hiep, L. Averyanov, N. S. Khang et al., LA-VN 1496 (HNL, FOF, LE). Northeastern Laos, Houaphan province, Hem district, Nacoc village, 12 April 2015, N. T. Hiep, L. Averyanov, N. S. Khang et al., LA-VN 1437 (HNL, FOF, LE). Northeastern Laos, Houaphan province, Viengxay district, Vieng Xai village, 7 April 2015, N. T. Hiep, L. Averyanov, 
N. S. Khang et al., LA-VN 1105 (HNL, FOF, LE). Northeastern Laos, Houaphan province, Xon district, Nong Hua Pa Noi village, 11 April 2015, N. T. Hiep, L. Averyanov, N. S. Khang et al., LA-VN 1247 (HNL, FOF, LE).

Epiphyte. Primary and secondary broad-leaved evergreen and semideciduous dry forests on rocky limestone, commonly on hill and mountain tops, 700-1450 m. Fl. March - May. Locally common.

S. China, Vietnam, Philippines. Laos: Houaphan, Xiangkhouang.

\section{L. zollingeri Rchb. f.,}

1863, Ann. Bot. Syst. 6: 622; Schuit. et al., 2008, Nord. Journ. Bot. 26: 297.

Northeastern Laos, Houaphan province, Viengxay district, Xieng Luong village, 8 April 2015, N. T. Hiep, L. Averyanov, N. S. Khang et al., $L A-V N 1154$ (HNL, FOF, LE).

Epiphyte. Primary and secondary broad-leaved evergreen forests on any kind of soil, preferably on rocky limestone, commonly on hill and mountain tops, 600-900 m. Fl. March - May. Not rare.

Andaman Islands, Thailand, Vietnam, Malacca Peninsula, Sumatra, Java, Borneo. Laos: Houaphan, Louang Namtha.

\section{Malleola Schltr.}

M. dentifera J. J. Sm.,

1927, Bull. Jard. Bot. Buitenzorg 3, 9: 191; Schuit. et al., 2008, Nord. Journ. Bot. 26: 298.

Northeastern Laos, Houaphan province, Viengxay district, Xieng Luong village, 8 April 2015, N. T. Hiep, L. Averyanov, N. S. Khang et al., LA-VN $1174 a /$ TM 1275 (LE).

Miniature epiphyte. Primary and secondary broad-leaved evergreen forests on rocky limestone, commonly on hill and mountain tops, 300-700 m. Fl. June - August.

Thailand, Vietnam, Malacca Peninsula, Sumatra, Borneo. Laos: Houaphan, Vientiane.

Nervilia Gaudich.

\section{N. aragoana Gaudich.}

1829, Voy. Uranie 422; Newman et al., 2007, Checklist Vasc. P1. Lao PDR: 374; Schuit. et al., 2008, Nord. Journ. Bot. 26: 299.

$=N$. fordii (Hance) Schltr., 1911, Bot. Jahrb. Syst. 45: 403.

$=N$. mekongensis $\mathrm{S} . \mathrm{W}$. Gale, Schuit. et Suddee, 2016, Phytotaxa 247, 4: 268.

Central Laos, Xiangkhouang province, Peak district, Khoang village, 3 April 2015, N. T. Hiep,
L. Averyanov, N. S. Khang et al., LA-VN 998 (HNL, FOF, LE).

Terrestrial tuberiferous ephemeroid herb. Open places in primary and secondary broad-leaved evergreen forests and woodlands, secondary scrub, pastures, secondary grasslands along agricultural fields and roads, preferably on lime soil, 170-1450 m. Fl. March - May. Not rare.

Tropical and subtropical Asia, tropical islands of western Pacific. Laos: Bolikhamxai, Champasak, Saravan, Vientiane, Xiangkhouang.

Widespread and extremely variable species expanding easy into various secondary anthropogenic habitats.

Oberonia Lindl.

o. cavaleriei Finet,

1908, Bull. Soc. Bot. France 55: 334, pl. 10, 1120; Aver., 2013, Turczaninowia 16, 4: 40.

Central Laos, Xiangkhouang province, Peak district, Khoang village, 3 April 2015, N. T. Hiep, L. Averyanov, N. S. Khang et al., LA-VN 964 (HNL, FOF, LE). Northeastern Laos, Houaphan province, Hem district, Na Puok village, 11 April 2015, N. T. Hiep, L. Averyanov, N. S. Khang et al., LA-VN 1308 (HNL, FOF, LE). Northeastern Laos, Houaphan province, Viengxay district, Vieng Xai village, 7 April 2015, N. T. Hiep, L. Averyanov, N. S. Khang et al., LA-VN 1130 (HNL, FOF, LE). Northeastern Laos, Houaphan province, Viengxay district, Xieng Luong village, 8 April 2015, N. T. Hiep, L. Averyanov, N. S. Khang et al., LA-VN 1159 (HNL, FOF, LE).

Lithophyte. Primary and secondary broad-leaved and mixed evergreen forests on rocky limestone, commonly on shady vertical cliffs near hill and mountain tops, 500-1450 m. Fl. January - May. Not common.

NE. India, Nepal, Myanmar, S. China, Thailand, Vietnam. Laos: Houaphan, Vientiane, Xiangkhouang.

O. ensiformis Lindl.,

1859, Fol. Orch. Oberonia: 4; Newman et al., 2007, Checklist Vasc. Pl. Lao PDR: 275; Schuit. et al., 2008, Nord. Journ. Bot. 26: 300.

Central Laos, Xiangkhouang province, Peak district, Khoang village, 3 April 2015, N. T. Hiep, L. Averyanov, N. S. Khang et al., LA-VN 969 (HNL, FOF, LE). Northeastern Laos, Houaphan province, Hem district, Nacoc village, 12 April 2015, N. T. Hiep, $L$. Averyanov, N. S. Khang et al., LA-VN 1400 (HNL, FOF, LE). Northeastern Laos, Houaphan province, 
Viengxay district, Vieng Xai village, 7 April 2015, N. T. Hiep, L. Averyanov, N. S. Khang et al., LA-VN 1102 (HNL, FOF, LE).

Lithophyte and rarely epiphyte. Primary and secondary broad-leaved and mixed evergreen forests on rocky limestone, commonly on shady vertical cliffs near hill and mountain tops, 800-1450 m. Fl. September - April. Locally common.

India, Nepal, Bhutan, Myanmar, S. China, Thailand, Vietnam. Laos: Champasak, Houaphan, Vientiane, Xiangkhouang.

\section{O. gammiei King et Pantl.,}

1897, Journ. As. Soc. Bengal 66, 2: 578; Schuit. et al., 2008, Nord. Journ. Bot. 26: 300.

Central Laos, Vientiane province, Kasi town area, 25 November 2014, E. Konstantinov, K-291 (LE). Central Laos, Vientiane province, Vang Vieng district, Nathong village, 14 March 2013, N. T. Hiep, L. Averyanov, N. S. Khang et al., LA-VN 424 (HNL, FOF, LE). Northeastern Laos, Houaphan province, Hem district, Nacoc village, 12 April 2015, N. T. Hiep, L. Averyanov, N. S. Khang et al., LA-VN 1423 (HNL, FOF, LE).

Miniature epiphyte. Primary and secondary broad-leaved evergreen and deciduous forests on any kind of soil, preferably on rocky limestone, 3001200 m. Fl. September - November. Not common.

NE. India, Myanmar, S. China, Hainan, Thailand, Vietnam. Laos: Houaphan, Khammouan, Louangphrabang, Vientiane.

${ }^{\mathrm{L}} \boldsymbol{O}$. kwangsiensis Seidenf., 1968, Dansk Bot. Ark., 25, 3: 31, fig. 14.

Central Laos, Xiangkhouang province, Peak district, Khoang village, 3 April 2015, N. T. Hiep, L. Averyanov, N. S. Khang et al., LA-VN 990 (HNL, FOF, LE). Northeastern Laos, Houaphan province, Xon district, Nong Hua Pa Noi village, 11 April 2015, N. T. Hiep, L. Averyanov, N. S. Khang et al., $L A-V N 1252 a$ (LE).

Lithophyte and epiphyte. Primary and secondary broad-leaved evergreen forests on rocky limestone, commonly in shady mossy places near hill and mountain tops, 1200-1450 m. Fl. October - November. Rare.

S. China, Thailand, Vietnam. Laos: Houaphan, Xiangkhouang.

${ }^{\mathrm{L}}$ O. maxima Hook. f.,

1888, Fl. Brit. India 5: 677.

Fig. 10B, C.

Northeastern Laos, Houaphan province, Xon district, Nong Hua Pa Noi village, 11 April 2015,
N. T. Hiep, L. Averyanov, N. S. Khang et al., LA-VN 1246 / TM 1284, LA-VN 1274 (HNL, FOF, LE).

Epiphyte. Primary and secondary broad-leaved evergreen dry forests on rocky shale and limestone, 1100-1200 m. Fl. August - October. Rare.

NE. India, Myanmar, N. Thailand. Laos: Houaphan.

O. mucronata (D. Don) Ormerod et Seidenf.,

1997, Contrib. Orch. Fl. Thailand 13: 20; Newman et al., 2007, Checklist Vasc. P1. Lao PDR: 275; Schuit. et al., 2008, Nord. Journ. Bot. 26: 300.

$=$ Oberonia iridifolia Lindl., 1830, Gen. Sp. Orch. Pl.: 15.

Northeastern Laos, Houaphan province, Hem district, Khon Ngua village, 15 April 2015, N. T. Hiep, L. Averyanov, N. S. Khang et al., LA-VN 1468 (HNL, FOF, LE).

Epiphyte. Primary and secondary broad-leaved evergreen and deciduous open forests on any kind of soil, often on rocky limestone, 170-1050 m. Fl. September - October. Not common.

India, Nepal, Bhutan, Bangladesh, Myanmar, S. China, Thailand, Malacca Peninsula, Philippines, Indonesia, islands of western Pacific. Laos: Bolikhamxai, Champasak, Houaphan, Khammouan, Phongsali, Vientiane.

\section{O. simplex Aver., sp. nov.}

Described from southern Cambodia ("Kampot province, Phnom Bokor national park, $10^{\circ} 37.923 \mathrm{~N}$, $104^{\circ} 01.143 \mathrm{E}$, epiphyte in cloud short forest along small stream at elevation about $1000 \mathrm{~m}$ a.s.l."). Type ("16 December 2014, E. Konstantinov K-369) LE (holotype).

Fig. 9; 10D, E.

Species epithet refers simple, unlobed, rectangular-ligulate flower lip.

Perennial sympodial epiphytic miniature herb 1-2(2.5) $\mathrm{cm}$ tall. Stem abbreviate, very short, 1.52(3) $\mathrm{mm}$ long, completely covered by (3)4-6(7) imbricate leaves, with many thin, flexuose, dull yellowish gray roots at the base. Leaves distichous, fleshy, with more or less distinct articulation at the base, lanceolate, slightly oblique, triangular obtuse at apex, (0.6)1-1.8(2.2) cm long, (1.2)1.5-2.2(2.6) $\mathrm{mm}$ wide. Inflorescence subterminal, arching to almost straight raceme, (1.5)2-4(4.5) cm long, 2-3 $\mathrm{mm}$ in diam.; scape stout, erect, straight or slightly arching, (0.4)0.6-1.5(2) cm long, covered by many spirally arranged, overlapping, narrowly lanceolate to almost filiform aristate sterile bracts $1.5-2 \mathrm{~mm}$ long; rachis arching to almost straight, more or less 
rigid, finely longitudinally ribbed, $(0.8) 1-2(2.5) \mathrm{cm}$ long, $0.4-0.5 \mathrm{~mm}$ in diam. Floral bracts in basal part of raceme narrowly triangular, (0.8)1-1.2(1.4) $\mathrm{mm}, 0.2-0.3 \mathrm{~mm}$ wide, to the apex broadly ovate triangular, concave, (0.5)0.6-0.8(1) $\mathrm{mm}$ long by $0.5-0.7 \mathrm{~mm}$, shortly acuminate, incise irregularly denticulate along the margin, during anthesis more or less recurved. Pedicel and ovary $0.4-0.5 \mathrm{~mm}$ long; pedicel shortly cylindric $0.2-0.25 \mathrm{~mm}$ long, about $0.1 \mathrm{~mm}$ in diam.; ovary narrowly obovoid, $0.2-0.25$ $\mathrm{mm}$ long, $0.2-0.3 \mathrm{~mm}$ broad; developing ovary and ripening fruit obovoid, (0.5)1-1.2 mm long, 0.6-0.8 $\mathrm{mm}$ in diam., longitudinally ribbed. Flowers $(0.9) 1-$ $1.2(1.3) \mathrm{mm}$ across, white to slightly yellowish.
Sepals broadly ovate, $0.6-0.7 \mathrm{~mm}$ long, $0.4-0.5$ $\mathrm{mm}$ wide, concave, obtuse, slightly cucullate, with entire, often revolute margin. Petals narrowly lanceolate to almost linear, acute, straight and entire along the margin. Lip rather fleshy, simple, unlobed, oblong rectangular, slightly broadening to the base, truncate at apex, $0.6-0.7 \mathrm{~mm}$ long, $0.2-0.3 \mathrm{~mm}$ wide, straight along margin, at the base with shallow hemi orbicular fovea.

Miniature canopy and branch epiphyte. Submontane broad-leaved primary and old secondary humid cloud forest on granite, usually along small streams, 1000 m. Fl. February December. Very rare.
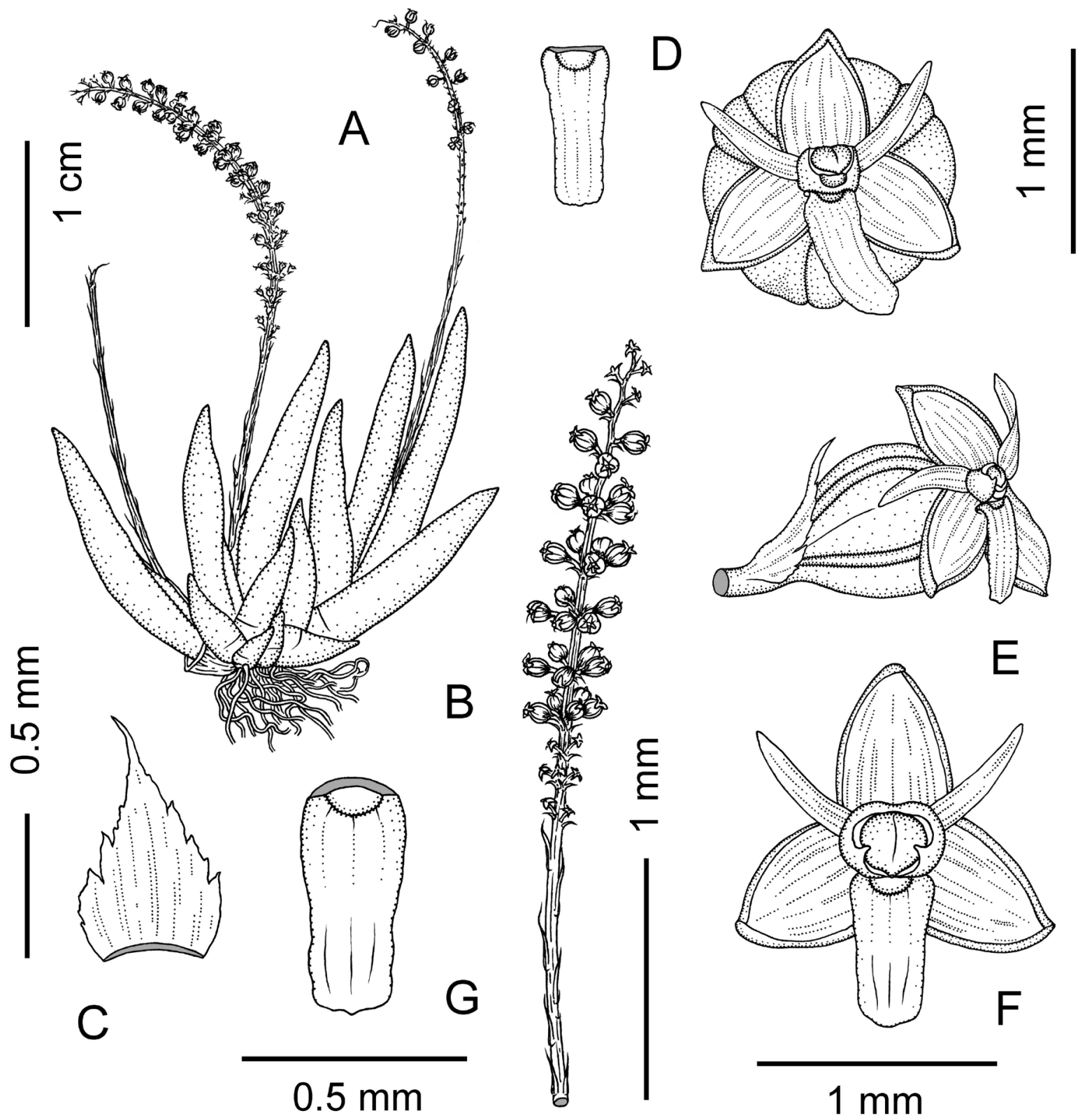

Fig. 9. New and rare orchids in the flora of Cambodia and Laos. Oberonia simplex Aver. A - flowering and fruiting plant; B - inflorescence; C - floral bract; D - flower and lip at fruiting stage, half side and frontal view; E - flower at fruiting stage, side view; $\mathrm{F}$ - flower, frontal view; $\mathrm{G}$ - lip, frontal view. All drawn from the type specimen $(E$. Konstantinov K-369) by L. Averyanov and T. Maisak. 
Cambodia: Kampot. Endemic.

Species differs from all its known congeners by ligulate unlobed lip, which represents unique character in the genus.

\section{Odontochilus Blume}

O. tortus King et Pantl.,

1896, Journ. Asiat. Soc. Bengal, Pt. 2, Nat. Hist. 65: 125.

$\equiv$ Anoectochilus tortus (King et Pantl.) King et Pantl., 1898, Ann. Roy. Bot. Gard. (Calcutta) 8: 298; Schuit. et al., 2008, Nord. Journ. Bot. 26: 264.

= Odontochilus repens Downie, 1925, Bull.

Misc. Inform. Kew 1925: 413.

$\equiv$ Pristiglottis torta (King et Pantl.) Aver., 1996, Bot. Journ. (Moscow \& St. Petersburg) 81, 10: 78.

Central Laos, Vientiane province, Kasi town area, Nam Pong Noi River along new road to Louangphrabang town, $13 \mathrm{~km}$ from Kasi town, 1 December 2014, E. Konstantinov, K-308 (LE).

Terrestrial creeping herb. Primary and secondary evergreen broad-leaved shady forests, commonly on humid river valley slopes, 800-1300. Fl. NovemberDecember. Rare.

N. India, Bhutan, S. China, Thailand, Vietnam. Laos: Champasak, Vientiane.

Panisea (Lindl.) Lindl.

P. tricallosa Rolfe,

1901, Bull. Misc. Inform. Kew 1901: 148; Newman et al., 2007, Checklist Vasc. P1. Lao PDR: 276; Schuit. et al., 2008, Nord. Journ. Bot. 26: 301.

Central Laos, Xiangkhouang province, Peak district, Khoang village, 3 April 2015, N. T. Hiep, L. Averyanov, N. S. Khang et al., LA-VN 956 (HNL, FOF, LE).

Epiphyte and lithophyte. Primary and secondary broad-leaved evergreen forests, usually on rocky limestone, 1200-1500 m. Fl. March - May. Not common.

NE. India, Nepal, Thailand, S. China, Vietnam. Laos: Xaisomboun.

\section{P. uniflora (Lindl.) Lindl.,}

1854, Fol. Orchid. 5: 2; Newman et al., 2007, Checklist Vasc. Pl. Lao PDR: 276; Schuit. et al., 2008, Nord. Journ. Bot. 26: 301; Aver., 2013, Turczaninowia 16, 4: 40.

Laos, Khammouan province, Laksao town area, 31 November 2013, E. Konstantinov, K-219 (LE).

Epiphyte and lithophyte. Primary and secondary broad-leaved evergreen and deciduous forests, usually in gallery forests and in forests on rocky limestone, 400-1500 m. Fl. March - May, August, October. Not rare.

NE. India, Nepal, Bhutan, Myanmar, Thailand, Cambodia, Vietnam. Laos: Bolikhamxai, Bolikhamxai, Khammouan, Vientiane.

\section{Paphiopedilum Pfitzer}

P. concolor (Bateman) Pfitzer,

1888, Nat. Pflanzenfam. 2, 6: 84; Newman et al., 2007, Checklist Vasc. Pl. Lao PDR: 276; Schuit. et al., 2008, Nord. Journ. Bot. 26: 302; Aver., 2013, Turczaninowia 16, 4: 41.

Central Laos, Xiangkhouang province, Kham district, Huad village, 4 April 2015, N. T. Hiep, L. Averyanov, N. S. Khang et al., LA-VN 1048 (HNL, FOF, LE). Central Laos, Xiangkhouang province, Peak district, Khoang village, 3 April 2015, N. T. Hiep, L. Averyanov, N. S. Khang et al., LA-VN 967 (HNL, FOF, LE). Northeastern Laos, Houaphan province, Hem district, Na Puok village, 11 April 2015, N. T. Hiep, L. Averyanov, N. S. Khang et al., $L A-V N 1310$ (HNL, FOF, LE). Northeastern Laos, Houaphan province, Hem district, Nacoc village, 12 April 2015, N. T. Hiep, L. Averyanov, N. S. Khang et al., LA-VN 1401 (HNL, FOF, LE). Northeastern Laos, Houaphan province, Xon district, Nong Hua Pa Noi village, 11 April 2015, N. T. Hiep, L. Averyanov, N. S. Khang et al., LA-VN 1277 (HNL, FOF, LE).

Lithophyte. Primary and secondary broad-leaved evergreen and semideciduous forests on rocky limestone, commonly near hill and mountain tops, (200)500-1450 m. Fl. March - May, occasionally flowers throughout the year. Locally common. Ornamental.

Myanmar, S. China, Thailand, Vietnam, Cambodia. Laos: Bolikhamxai, Champasak, Houaphan, Khammouan, Vientiane, Xiangkhouang.

P. dianthum Tang et F. T. Wang,

1940, Bull. Fan Mem. Inst. Biol. 10: 24; Schuit. et al., 2008, Nord. Journ. Bot. 26: 302; Aver., 2013, Turczaninowia 16, 4: 41.

Northeastern Laos, Houaphan province, Hem district, Nacoc village, 12 April 2015, N. T. Hiep, L. Averyanov, N. S. Khang et al., LA-VN 1443 (HNL, FOF, LE).

Lithophyte. Primary and secondary broad-leaved evergreen forests on rocky limestone, commonly near hill and mountain tops, $1000-1750 \mathrm{~m}$. Fl. September - November. Not common. Ornamental.

S. China, Vietnam. Laos: Bolikhamxai, Houaphan, Vientiane. 


\section{Pelatantheria Ridl.}

P. ctenoglossum Ridl.,

1896, Journ. Linn. Soc., Bot. 32: 372; Newman et al., 2007, Checklist Vasc. P1. Lao PDR: 277; Schuit. et al., 2008, Nord. Journ. Bot. 26: 303; Aver., 2013, Turczaninowia 16, 4: 41.

Central Laos, Khammouan province, Laksao town area, 31 October 2013, E. Konstantinov, $K-221$ (LE). Central Laos, Khammouan province, Thakhek town area, Pha In Cave, 3 November 2013, E. Konstantinov, $K-235, K-240$ (LE). Northeastern Laos, Houaphan province, Hem district, Khon Ngua village, 15 April 2015, N. T. Hiep, L. Averyanov, N. S. Khang et al., LA-VN 1472 (HNL, FOF, LE).

Epiphyte and lithophyte. Primary and secondary broad-leaved evergreen open dry forests on rocky limestone, commonly on hill and mountain tops, 200-950 m. Fl. May - August. Not rare.

Thailand, Vietnam, Cambodia. Laos: Champasak, Houaphan, Khammouan, Phongsali, Savannakhet, Vientiane.

\section{${ }^{\mathrm{L}}$ P. insectifera Ridl.,}

1896, Journ. Linn. Soc., Bot. 32: 373.

= Sarcanthus rivesii Guillaumin, 1930, Bull. Soc. Bot. France 77: 330.

$=S$. tonkinensis Guillaumin, 1930, Bull. Soc. Bot. France 77: 3111930.

$=S$. simondianus Gagnep., 1951, Bull. Mus. Natl. Hist. Nat. 2, 22: 630.

$=$ Pelatantheria rivesii (Guillaumin) Tang et $\mathrm{F} . \mathrm{T}$. Wang, 1951, Acta Phytotax. Sin. 1: 101; Newman et al., 2007, Checklist Vasc. P1. Lao PDR: 277; Schuit. et al., 2008, Nord. Journ. Bot. 26: 303.

Fig. 10, F.

Northeastern Laos, Houaphan province, Viengxay district, Vieng Xai village, 7 April 2015, N. T. Hiep, L. Averyanov, N. S. Khang et al., LA-VN 1142 (HNL, FOF, LE). Northeastern Laos, Houaphan province, Viengxay district, Xieng Luong village, 8 April 2015, N. T. Hiep, L. Averyanov, N. S. Khang et al., LA-VN 1165 (HNL, FOF, LE).

Epiphyte and lithophyte. Primary and secondary broad-leaved evergreen and semideciduous dry open forests on rocky limestone, commonly on hill and mountain tops, often on mossy exposed rocks, 600-1100 m. Fl. October - November. Locally abundant.

NE. India, Bangladesh, Andaman Islands, Myanmar, Thailand, S. China, Vietnam. Laos: Champasak, Houaphan, Louang Namtha, Phongsali.

The differences between $P$. insectifera and $P$. rivesii remains very unclear. It is obvious that last one hardly desires species status and may be recognized only as an "eastern" variety of even form of widespread and variable $P$. insectifera.

\section{Phaius Lour.}

P. flavus Lindl.,

1831, Gen. Sp. Orch. Pl.: 128; Newman et al., 2007, Checklist Vasc. P1. Lao PDR: 277; Schuit. et al., 2008, Nord. Journ. Bot. 26: 304; Aver., 2013, Turczaninowia 16, 4: 41.

Northeastern Laos, Houaphan province, Hem district, Na Puok village, 11 April 2015, N. T. Hiep, L. Averyanov, N. S. Khang et al., LA-VN 1350 (HNL, FOF, LE).

Terrestrial and occasionally lithophytic herb. Primary and secondary broad-leaved evergreen forests on any kind of soil, preferably on rocky limestone, usually in shady humid depressions on rich soil, 600-1200 m. Fl. March - June. Not common.

Tropical Asia and tropical islands of western Pacific. Laos: Houaphan, Louangphrabang, Vientiane.

\section{Phalaenopsis Blume}

${ }^{\mathrm{L}} \boldsymbol{P}$. braceana (Hook. f.) Christenson, 1986, Selbyana 9: 1691986.

Central Laos, Xiangkhouang province, Kham district, Huad village, 4 April 2015, N. T. Hiep, L. Averyanov, N. S. Khang et al., LA-VN 1054 (HNL, FOF, LE).

Epiphyte. Primary and secondary broadleaved evergreen open forests on rocky limestone, commonly on hill and mountain tops, $1200-1400 \mathrm{~m}$. Fl. April - June. Rare. Ornamental.

Bhutan, SW. China (Yunnan), N. Vietnam. Laos: Xiangkhouang.

\section{Pholidota Lindl.}

\section{P. articulata Lindl.,}

1830, Gen. Sp. Orch. P1.: 38; Newman et al., 2007, Checklist Vasc. P1. Lao PDR: 277; Schuit. et al., 2008, Nord. Journ. Bot. 26: 305; Aver., 2013, Turczaninowia 16, 4: 41.

Central Laos, Xiangkhouang province, Peak district, Khoang village, 3 April 2015, N. T. Hiep, L. Averyanov, N. S. Khang et al., LA-VN 984 (HNL, FOF, LE). Northeastern Laos, Houaphan province, Hem district, Na Puok village, 11 April 2015, N. T. Hiep, L. Averyanov, N. S. Khang et al., LA-VN 1298 (HNL, FOF, LE). Northeastern Laos, Houaphan province, Hem district, Nacoc village, 12 April 2015, N. T. Hiep, L. Averyanov, N. S. Khang et al., LA-VN 1434 (HNL, FOF, LE). Northeastern Laos, Houaphan province, Viengxay district, Vieng Xai 


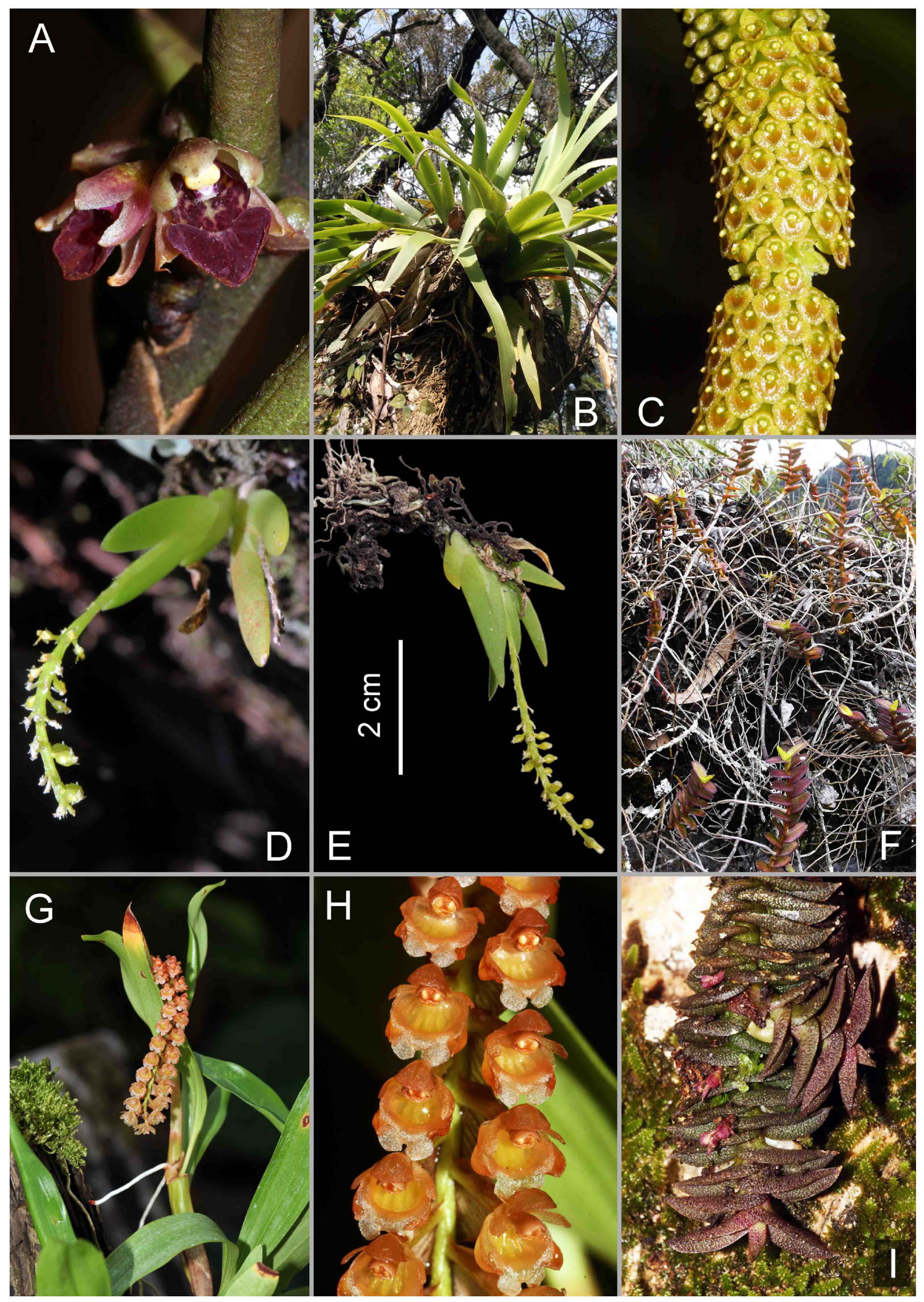

Fig. 10. New and rare orchids in the flora of Cambodia and Laos. A - Luisia ramosii Ames (LA-VN 1098). B, C Oberonia maxima Hook. f. (LA-VN 1246 / TM 1284). F - Pelatantheria insectifera Ridl. (LA-VN 1142). G, H P. recurva Lindl. (T. Maisak et al., 907). I - Schoenorchis scolopendria Aver. (LA-VN 1174). Photos by L. Averyanov (A, C, F-I), T. Maisak (B) and E. Konstantinov (D, E). 
village, 7 April 2015, N. T. Hiep, L. Averyanov, N. S. Khang et al., LA-VN 1143 (HNL, FOF, LE). Northeastern Laos, Houaphan province, Xon district, Nong Hua Pa Noi village, 11 April 2015, N. T. Hiep, L. Averyanov, N. S. Khang et al., LA-VN 1248 (HNL, FOF, LE).

Creeping epiphyte and occasionally lithophyte. Primary and secondary broad-leaved, mixed and coniferous evergreen and deciduous forests and woodlands on any kind of soil, preferably on rocky limestone, mostly on hill and mountain tops, (450) 500-1750 m. Fl. June - August. Not rare.

India, Nepal, Bhutan, Myanmar, SW. China (Yunnan), Thailand, Vietnam, Cambodia, Malacca Peninsula, Sumatra, Java, Kalimantan, Sulawesi, Philippines. Laos: Attapu, Bolikhamxai, Champasak, Houaphan, Khammouan, Louang Namtha, Louangphrabang, Phongsali, Saravan, Vientiane, Xiangkhouang.

\section{P. imbricata Lindl.,}

1825, Exot. Fl. 2, t. 138; Newman et al., 2007, Checklist Vasc. P1. Lao PDR: 278; Schuit. et al., 2008, Nord. Journ. Bot. 26: 306; Aver., 2013, Turczaninowia 16, 4: 41

Northeastern Laos, Houaphan province, Hem district, Na Puok village, 11 April 2015, N. T. Hiep, L. Averyanov, N. S. Khang et al., LA-VN 1302 (HNL, FOF, LE). Northeastern Laos, Houaphan province, Hem district, Nacoc village, 12 April 2015, N. T. Hiep, L. Averyanov, N. S. Khang et al., LA-VN 1394 (HNL, FOF, LE). Northeastern Laos, Houaphan province, Viengxay district, Vieng Xai village, 7 April 2015, N. T. Hiep, L. Averyanov, N. S. Khang et al., LA-VN 1136 (HNL, FOF, LE). Northwestern Laos, Xiangkhouang province, Ban Thathom town area, 26 October 2013, E. Konstantinov, K-169(LE).

Epiphyte and lithophyte. Primary and secondary broad-leaved, mixed and coniferous evergreen and deciduous forests and woodlands on any kind of soil, preferably on rocky limestone, mostly on hill and mountain tops, 200-1200 m. Fl. June - August. Not rare.

Tropical Asia, Australia, tropical Pacific islands. Laos: Attapu, Bolikhamxai, Champasak, Houaphan, Houaphan, Khammouan, Louang Namtha, Phongsali, Vientiane, Xaisomboun, Xiangkhouang.

\section{P. pallida Lindl.,}

1835, Bot. Reg. 21, t. 1777; Newman et al., 2007, Checklist Vasc. Pl. Lao PDR: 278; Schuit. et al., 2008, Nord. Journ. Bot. 26: 306; Aver., 2013, Turczaninowia 16, 4: 41.
Central Laos, Xiangkhouang province, Kham district, Huad village, 4 April 2015, N. T. Hiep, L. Averyanov, N. S. Khang et al., LA-VN 1066 (HNL, FOF, LE). Central Laos, Xiangkhouang province, Peak district, Khoang village, 3 April 2015, N. T. Hiep, L. Averyanov, N. S. Khang et al., LA-VN 959 (HNL, FOF, LE).

Epiphyte and lithophyte. Primary and secondary broad-leaved and mixed evergreen and semideciduous forests on rocky limestone, mostly on hill and mountain tops, $1200-1750 \mathrm{~m}$. Fl. June August. Rare.

India, Nepal, Bhutan, Myanmar, SW. China (Yunnan), Thailand, Vietnam. Laos: Xiangkhouang, Vientiane.

${ }^{\mathrm{C}} \boldsymbol{P}$. recurva Lindl., 1830, Gen. Sp. Orch. P1.: 37.

Fig. 10G, H.

Southern Cambodia, about 16 kilometers to the N of Sihanoukville town, Khan Prey Nup district, Kbal Chhay Waterfall, 5 December 2012, T. Maisak, L. Osinovetz, M. Telepova, 907 (LE).

Creeping epiphyte. Primary and secondary evergreen, broad-leaved dry lowland forests on low hills. Fl. March - April. Not common.

NE. India, Nepal, Bhutan, Myanmar, Thailand, Vietnam, Laos. Cambodia: Sihanoukville.

\section{${ }^{\mathrm{L}} \boldsymbol{P}$. yunnanensis Rolfe,}

1903, Journ. Linn. Soc., Bot. 36: 24.

Northeastern Laos, Houaphan province, Viengxay district, Vieng Xai village, 7 April 2015, N. T. Hiep, L. Averyanov, N. S. Khang et al., LA-VN 1140 (HNL, FOF, LE).

Creeping lithophyte and epiphyte. Primary and secondary broad-leaved evergreen open forests on rocky limestone, commonly on hill and mountain tops, 800-950 m. Fl. September - October. Not common.

S. China, N Vietnam. Laos: Houaphan.

\section{Podochilus Blume}

\section{P. oxystophylloides Ormerod,}

2003, Taiwania 48: 143; Aver., 2013, Turczaninowia 16, 4: 42.

Central Laos, Xiangkhouang province, Peak district, Khoang village, 3 April 2015, N. T. Hiep, L. Averyanov, N. S. Khang et al., LA-VN 963 (HNL, FOF, LE). Northeastern Laos, Houaphan province, Hem district, Nacoc village, 12 April 2015, N. T. Hiep, L. Averyanov, N. S. Khang et al., LA-VN 1397 (HNL, FOF, LE). 
Lithophyte. Primary and secondary broad-leaved and mixed evergreen forests on rocky limestone, commonly on shady vertical cliffs near hill and mountain tops, $1200-1750$ m. Fl. August - September. Rare.

S. China, Vietnam. Laos: Houaphan, Vientiane, Xiangkhouang.

\section{Polystachya Hook.}

P. concreta (Jacq.) Garay et H. R. Sweet, 1974, Orquideologia 9: 206; Newman et al., 2007, Checklist Vasc. Pl. Lao PDR: 278; Schuit. et al., 2008, Nord. Journ. Bot. 26: 306.

Central Laos, Xiangkhouang province, Kham district, Huad village, 4 April 2015, N. T. Hiep, L. Averyanov, N. S. Khang et al., LA-VN 1071 (HNL, FOF, LE). Central Laos, Xiangkhouang province, Peak district, Khoang village, 3 April 2015, N. T. Hiep, L. Averyanov, N. S. Khang et al., LA-VN 999 (HNL, FOF, LE). Northeastern Laos, Houaphan province, Xon district, Nong Hua Pa Noi village, 11 April 2015, N. T. Hiep, L. Averyanov, N. S. Khang et al., LA-VN 1284 (HNL, FOF, LE).

Epiphyte. Primary and secondary broad-leaved and coniferous evergreen and deciduous forests, woodlands and thickets, on any kind of soil, preferably on rocky limestone, 400-1450 m. Fl. June September. Occasional.

Tropical areas all over the World. Laos: Champasak, Houaphan, Khammouan, Louang Namtha, Louangphrabang, Vientiane, Xiangkhouang.

\section{Pomatocalpa Breda}

\section{P. spicatum Breda,}

1829, Gen. Sp. Orch. Asclep. 3, t. 15; Newman et al., 2007, Checklist Vasc. P1. Lao PDR: 279; Schuit. et al., 2008, Nord. Journ. Bot. 26: 307.

Central Laos, Xiangkhouang province, Peak district, Khoang village, 3 April 2015, N. T. Hiep, L. Averyanov, N. S. Khang et al., LA-VN 965 (HNL, FOF, LE). Northeastern Laos, Houaphan province, Hem district, Na Puok village, 11 April 2015, N. T. Hiep, L. Averyanov, N. S. Khang et al., LA-VN 1292 (HNL, FOF, LE).

Epiphyte. Primary and secondary broad-leaved and mixed evergreen forests on any kind of soil, often along streams and on rocky limestone, 200-1450 m. Fl. February - April. Occasional.

Thailand, Vietnam, Malacca Peninsula, Sumatra, Java, Borneo, Lesser Sunda Islands, Philippines. Laos: Champasak, Houaphan, Houaphan, Louangphrabang, Phongsali, Vientiane, Xaisomboun, Xiangkhouang.

\section{Porpax Lindl.}

P. capuccinorum Aver., sp. nov.

Described fromsouthwesternLaos("Khammouan prov., Thakhek district, Thakhek town area, near Pha Cave, branch epiphyte in canopy of lone tree 8-10 м tall, remnants of lowland broad-leaved woodlands at elev. about $156 \mathrm{~m}$ a.s.l. around point $17^{\circ} 29.540 \mathrm{~N}, 105^{\circ} 09.917 \mathrm{E}$ "). Type ("3 November 2013, E. Konstantinov K-239.") - LE (holotype). Epitype - d-EXSICCATES OF VIETNAMESE FLORA $0248 / K 239$.

Fig. $11 ; 12$ (d-EXSICCATES OF VIETNAMESE FLORA 0248/K 239).

Species epithet refers resemblance of the flowers with traditional capuchin monk wear.

Perennial short creping or clustering sympodial miniature epiphytic herb. Rhizome short, tiny, negligible. Stems pseudobulbous, densely touching each other, 2-leaved; young stems, grassy-green, enveloped by whitish or greenish, scarious, hyaline leaf sheaths; mature pseudobulbs fleshy, dull olive-green, flattened, oblate to discoid-lenticular, (5)7-1(1.2) cm across, (2)2.5-3(3.5) mm tall, early deciduous, covered by light brownish scarious sheath in form of membrane with prominent nerves disintegrating later into persistent fibrous remnants. Leaves 2, appearing in individual stem before flowering, subsessile, sheathed; sheath tubular, later much broadening, enveloping pseudobulb; leaf blade conduplicate, with distinct median vein, horizontally recurved, bright green, rather thin, herbaceous, narrowly obovate, (1) $1.5-2(2.2) \mathrm{cm}$ long, (4.5)5-7(7.5) mm wide, obtuse, narrowing into short petiole-like base 1.5-2 mm long. Inflorescence one per stem, hysteranthous, arising from the apex of mature leafless pseudobulb, 1-flowered; scape, pedicel and ovary, light dull green, (3.5)4-5(5.5) $\mathrm{mm}$ long, 0.8-1(1.2) $\mathrm{mm}$ in diam., bent at apex; peduncle with 3-4 sterile and 1 floral bracts. Scape and floral bracts, large, scarious, hyaline, light brownish or yellowish, broadly triangular, tubular at the base, shortly acuminate at apex, (2)3-5(5.5) mm long, being flattened (2)2.5-6(6.5) mm wide. Pedicel and ovary shortly cylindrical, (0.8)1-1.2(1.4) mm long. Flowers horizontally spreading, not widely opening, entirely bright red to red-brown, glabrous, (4)5-7(8) $\mathrm{mm}$ across. Sepals forward directed; median sepal narrowly ovate to ovate, (5)5.5-6(6.5) $\mathrm{mm}$ long, (2.8)3-3.2(3.5) mm wide, apically recurved, shortly attenuate, joined in lower half with lateral sepals; lateral sepals oblique falcate, strongly bent down, concave, (7)7.5-8(8.5) mm long, (2.4)2.6-2.8(3) $\mathrm{mm}$ wide, round and apiculate at apex, joined each 

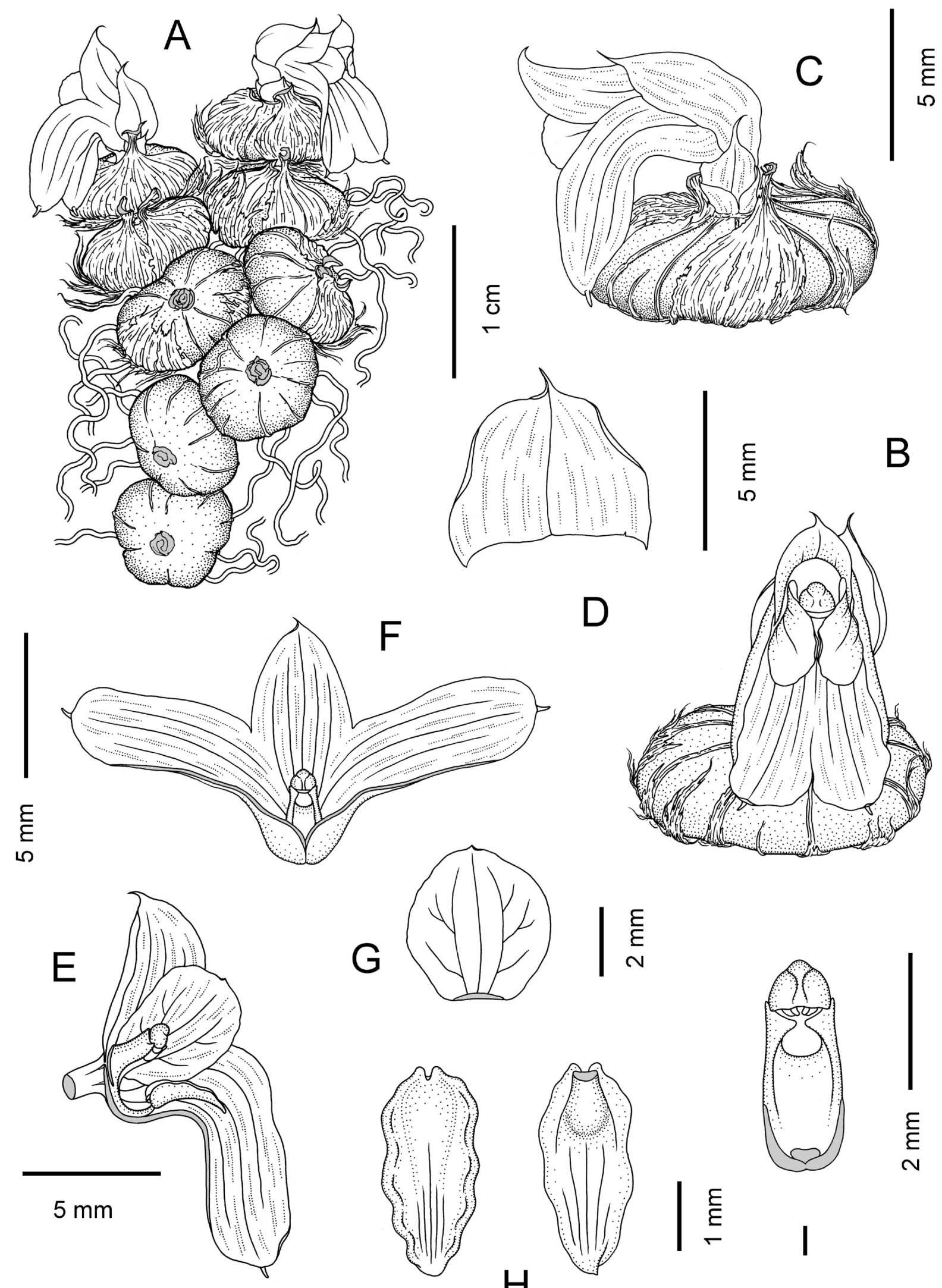

D
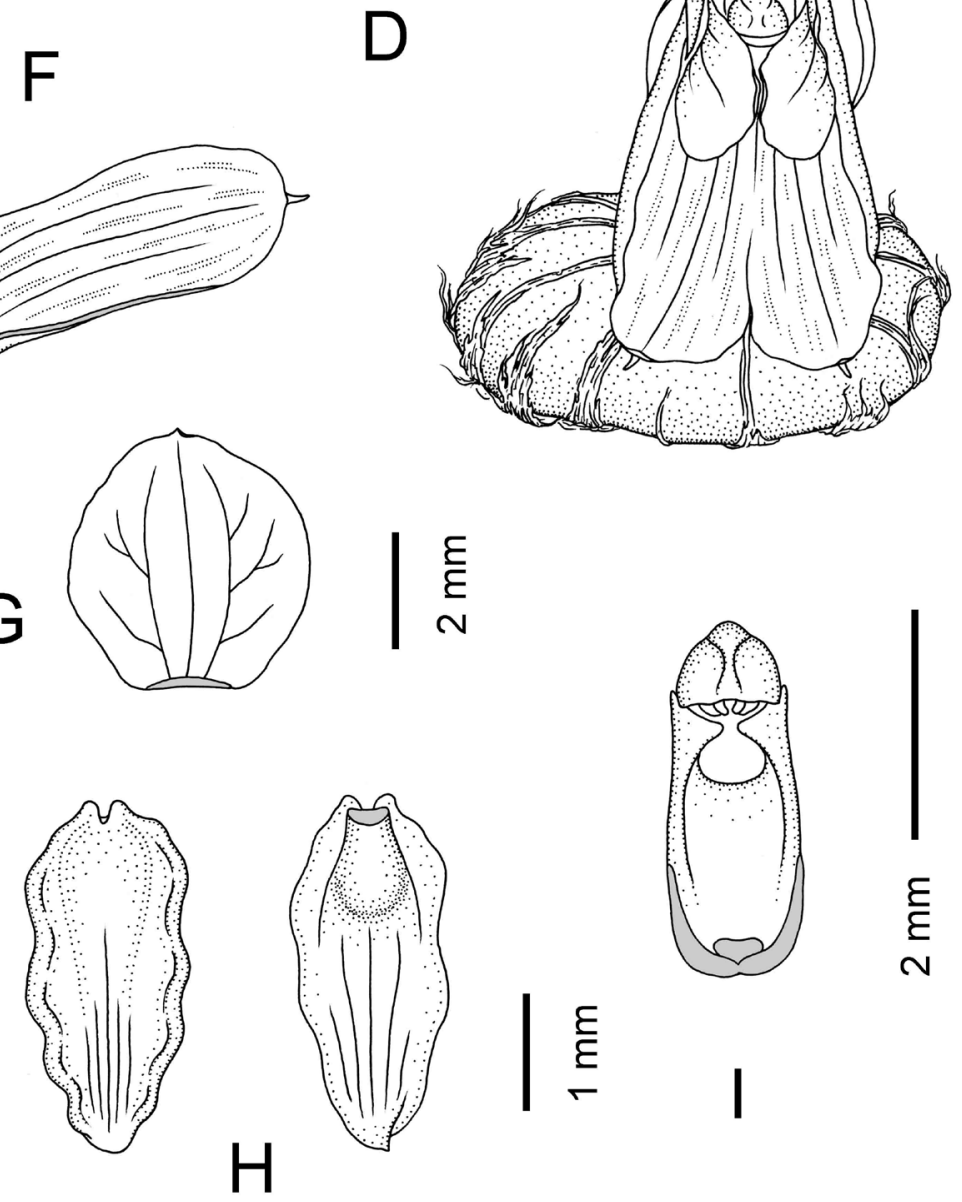

Fig. 11. New and rare orchids in the flora of Cambodia and Laos. Porpax capuccinorum Aver. A - Flowering plant. B Flower and flowering pseudobulb, frontal view. C - Flower and flowering pseudobulb, side view. D - Floral bract. E Flower with lateral sepal and petal removed, side view. F - Flattened sepals and column, frontal view. G - Flattened petal. $\mathrm{H}-$ Flattened lip, view from above and from below. I - Column, frontal view. All drawn from the type $-K-239$ by L. Averyanov and T. Maisak. 


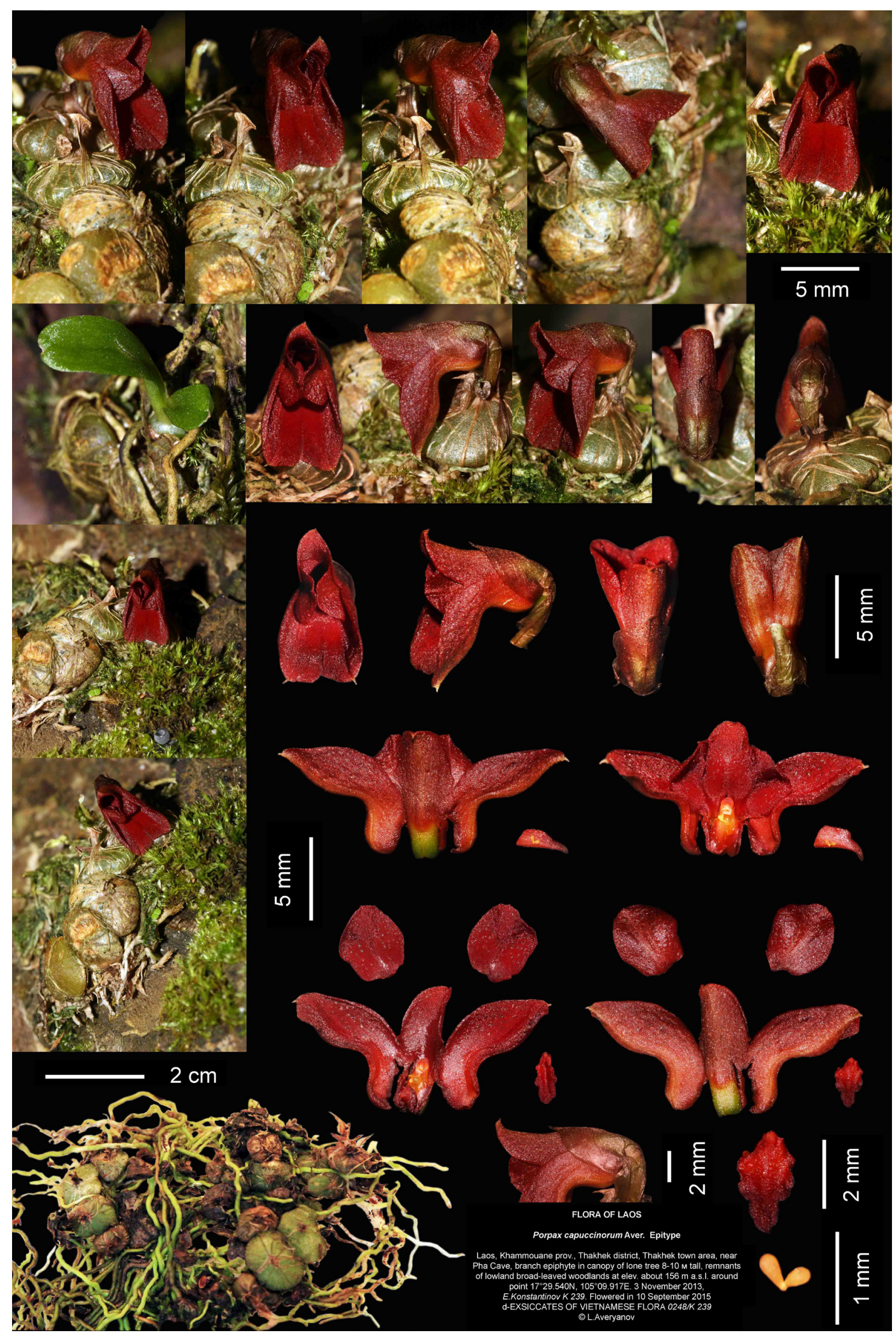

Fig. 12. New and rare orchids in the flora of Cambodia and Laos. Porpax capuccinorum Aver. Digital epitype - dEXSICCATES OF VIETNAMESE FLORA $0248 / K$ 248. Photos of E. Konstantinov and L. Averyanov, design and image correction by L. Averyanov. 
other almost along all their length, forming at the base short, broad mentum (1.8)2-2.2(2.4) $\mathrm{mm}$ long and broad. Petals free, broadly obovate to almost round, finely irregularly crenulate along the margin, (3.8)4-4.2(4.4) $\mathrm{mm}$ across, somewhat incurved closing entrance to the flower. Lip simple, somewhat movable, entire, narrowly ovate, (2.8)3-3.2(3.4) $\mathrm{mm}$ long, (1.3)1.5-1.6(1.7) $\mathrm{mm}$ wide, glabrous, slightly recurved, narrowing to blunt apex, with no ornamentation, straight or slightly undulate along the margin, at the base with flat fleshy callus notched adaxially. Column red to orange-red, broad, erect, stout, shortly cylindric, (2.6)2.8-3(3.2) mm tall, (1.4)1.6-1.8(2) mm wide, on sides with small, insignificant, erect, acute stelidia; stigma concave; rostellum in form of small, thin, truncate plate; column foot short, thick, concave, $1 \mathrm{~mm}$ long, as wide as column base, slightly forward curved forming at apex short narrow stalk joined with lip base. Anther cap bright red, hemispheric, smooth, $0.8-1 \mathrm{~mm}$ tall and wide, truncate at front. Pollinia 8 , yellow, in two groups, clavate, $0.2-0.3 \mathrm{~mm}$ long.

Miniature branch and trunk epiphyte. Primary and secondary dry semideciduous broad-leaved lowland forests on alluvial soils derived from limestone, 150-200 m. Fl. August - September. Rare.

Laos: Khammouan. Endemic.

Newly discovered plant belongs to group of species with hysteranthous inflorescence appearing from the apex of mature pseudobulb. From all representatives of this group new species strikingly differs in disproportionally long and broad lateral sepals (twice longer than median sepal) strongly bent down, almost round petals connivent with their apices at front of column and simple lip slightly undulate along the margin and notched at the base. Small population of this curious plant was found in canopy of lone remnant expiring tree remaining in almost completely deforested area, hence species should be regarded as crotically endangered.

P. elwesii (Rchb. f.) Rolfe,

1908, Orchid Rev. 16: 8; Newman et al., 2007, Checklist Vasc. Pl. Lao PDR: 279; Schuit. et al., 2008, Nord. Journ. Bot. 26: 307; Aver., 2013, Turczaninowia 16, 4: 42.

Central Laos, Vientiane province, Phou Khao Khouay National Protected Area to about $40 \mathrm{~km} \mathrm{NE}$ of Vientiane, 9 December 2015, L. Averyanov, T. Maisak, AL 153 (LE).

Miniature lithophyte and occasionally epiphyte. Primary and secondary evergreen broad-leaved forests along rocky streams and rivers, commonly in shady places on mossy boulders, 350-1500 m. Fl. October - December. Locally common.

India, Nepal, Bhutan, Vietnam, Malacca Peninsula. Laos: Bolikhamxai, Champasak, Khammouan, Vientiane.

\section{P. laosensis Aver., sp. nov.}

Described from central Laos ("Vientiane province, Phou Khao Khouay National Protected Area to about $40 \mathrm{~km}$ NE of Vientiane, dry evergreen primary and secondary forest along rocky stream on sandstone at elevation about $350 \mathrm{~m}$ a.s.1. ..."). Type ("9 December 2015, L. Averyanov, T. Maisak, AL 159") - LE (holotype). Epitype - d-EXSICCATES OF VIETNAMESE FLORA 0247/AL 159.

Fig. 13, 14 (d-EXSICCATES OF VIETNAMESE FLORA 0247/AL 159).

Species epithet refers country of its origin.

Perennial short creping or clustering sympodial miniature epiphytic or lithophytic herb. Rhizome short, tiny, negligible. Stems pseudobulbous, densely touching each other, 2-leaved; young shoot, arising from the base of mature pseudobulb, during anthesis grassy-green, (2)2.5-3(3.5) $\mathrm{mm}$ long, 1.5-2 $\mathrm{mm}$ in diam., enveloped by 3-4 white, scarious, hyaline, triangular-tubular bracts (3)3.5-5(5.5) $\mathrm{mm}$ long and wide (being flattened), bearing 2 juvenile sessile, cordate leaves (1.5)1.8-2.2(2.4) $\mathrm{mm}$ long and wide, and apical inflorescence; mature pseudobulbs fleshy, dull green, flattened, oblate to discoid-lenticular, broadly obovate of flabelliform in outline, (0.7)9$1.4(1.6) \mathrm{cm}$ across, (2.2)2.5-3.5(4) mm thick, early deciduous, covered by brownish scarious sheath in form of reticulate, cage-like membrane with prominent nerves disintegrating later into reticular fibrous remnants, oldest pseudobulbs almost naked. Leaves 2, sessile, developing in individual shoot after flowering, caducous to the end of pseudobulb formation. Inflorescence proteranthous, one per stem, arising from the apex of young shoot, 1-flowered; scape, pedicel and ovary, white to greenish, (2)2.5-3(3.5) $\mathrm{mm}$ long, $0.8-1 \mathrm{~mm}$ in diam., straight or slightly ascending. Floral bract, large, scarious, hyaline, white, broadly obovate to almost subquadrate, (3.6)3.8-4.2(4.4) mm long, (4) 4.2-4.5(4.7) mm wide, tubular, finely incise or denticulate along truncated apex, with more or less prominent central acute dent. Pedicel and ovary shortly cylindrical, (1.4)1.6-1.8(2) mm long, 1.2$1.5 \mathrm{~mm}$ in diam., glabrous or with few scarce scurfy hairs. Flowers horizontally spreading, entirely red-brown, glabrous, not widely opening, tubular, (1.8)2-2.2(2.4) $\mathrm{mm}$ across, (5)5.5-7(7.5) $\mathrm{mm}$ long. Sepals (5)5.5-6(6.5) long, forward directed, 

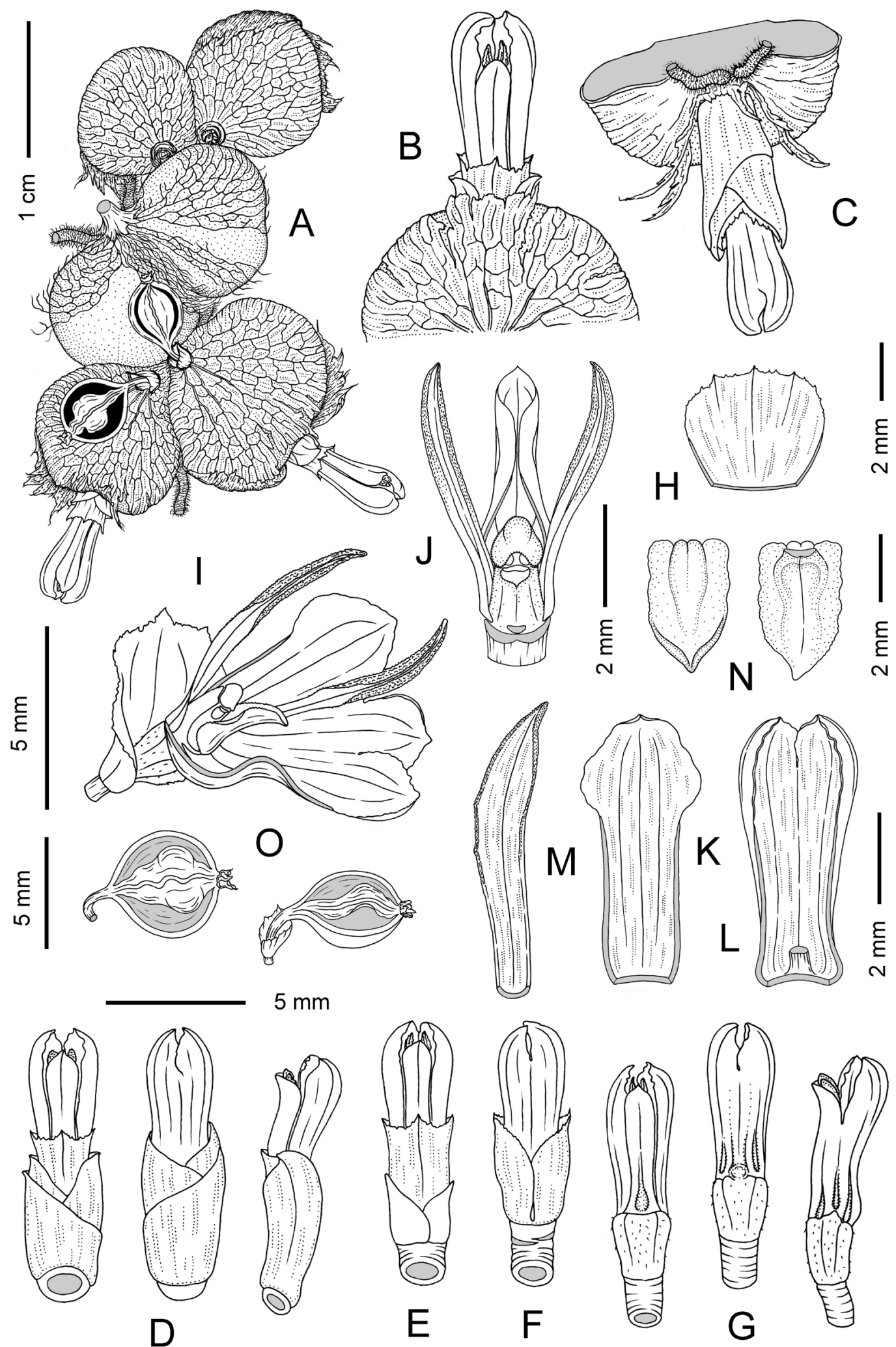

Fig. 13. New and rare orchids in the flora of Cambodia and Laos. Porpax laosensis Aver. A - Flowering and fruiting plant. B - Flower, view from above. C - Flower, view from below. D - Peduncle and flower with intact scape and floral bracts, views from below, from above and side view. E - Peduncle and flower with intact juvenile leaves and floral bract (scape bracts removed), view from below. F - Peduncle and flower with intact floral bract (scape bracts and juvenile leaves removed). G - Peduncle, pedicel, ovary and flower (scape bracts, juvenile leaves and floral bract removed), views from above, from below and side view. $\mathrm{H}$ - Flattened floral bract. I - Flattened flower, side view with section between median and lateral sepals. J - Flattened flower, with lateral sepals and lip removed, frontal view. K Flattened median sepal. L - Lateral sepals. M - Flattened petal. N - Flattened lip, views from above and from below. $\mathrm{O}-$ Fruit, view from above and half side view. All drawn from the type - AL 159 by L. Averyanov and T. Maisak. 


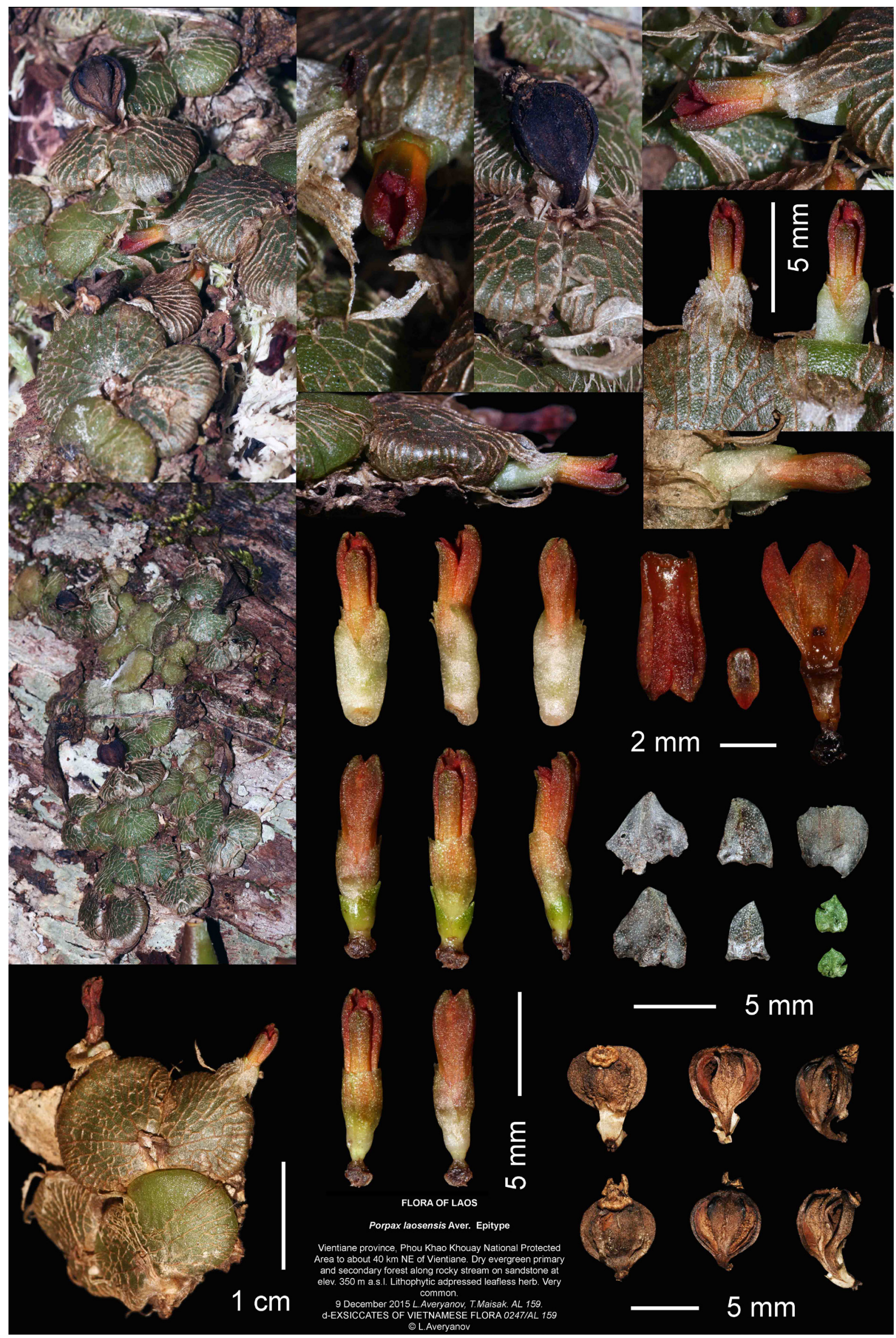

Fig. 14. New and rare orchids in the flora of Cambodia and Laos. Porpax laosensis Aver. Digital epitype - d-EXSICCATES OF VIETNAMESE FLORA 0247/AL 159. Photos, design and image correction by L. Averyanov. 
concave, united, forming tube; median sepal joined with lateral sepals on $2 / 3$ from the base, free part broadening, broadly ovate, (2)2.2-2.8(3) $\mathrm{mm}$ long and wide, obtuse, straight or finely undulate along the margin; lateral sepals cymbiform, straight, joined each other almost along all their length, with free lobes (0.8)1-1.5(1.6) mm long, (1.4)1.6-1.8(2) $\mathrm{mm}$ wide, finely undulate and cucullate at apex. Petals free, broadly lanceolate, oblique falcate, as long as sepals or little longer, $0.8-1.2 \mathrm{~mm}$ wide, acute, in apical half finely papillose and involute along the margin. Lip simple, somewhat movable, entire, rectangular with fleshy triangular apex, (3.2)2.5-3(3.2) mm long, (1.3)1.4-1.5(1.6) $\mathrm{mm}$ wide, glabrous, slightly recurved, at the base with flat insufficient callus obscurely notched adaxially, entire or finely crenulate along the margin, placed on short, narrow claw 0.5-0.6 mm long. Column red-brown, broad, erect, stout, shortly cylindric, (1.4)1.6-1.8(2) mm tall, (0.7)0.8-1(1.2) mm wide; at the apex from both sides with small triangular stelidia; stigma concave; rostellum in form of small, thin, truncate plate; column foot insufficient, in form of forward curved stalk joined to the lip base. Anther cap red-brown, broadly conoid, globular at the apex, smooth, $0.5-0.6 \mathrm{~mm}$ tall and wide, at front broadly truncate. Pollinia 8 , yellowish, in two groups, oblate, about $0.25 \mathrm{~mm}$ across. Fruit dark dirty brown to almost black, oblique, half-globular capsule (4.5)5-5.5(6) $\mathrm{mm}$ long and wide, ribbed on sides, opening by two splits on ventral flattened surface, placed on bent pedicel 1.5-2.5 mm long enveloped by scarious remnant of floral bract.

Miniature trunk epiphyte and lithophyte. Primary and secondary dry evergreen broad-leaved lowland forests on sandstone, commonly along streams and small rivers, 350-400 m. Fl. March - April. Locally common.

Laos: Vientiane. Endemic.

New plant belongs to group of species with proteranthous inflorescence appearing from the base of mature pseudobulb. From all species of this group new plant well differs in glabrous narrow flowers, free narrowly lanceolate finely papillulate petals involute along the margin and in short simple rectangular lip with acute-triangular apex. New species in its locus classicus was observed as common plant with large visually stable population.

Robiquetia Gaudich.

R. spathulata (Blume) J.J. Sm.,

1912, Natuurw. Tijdschr. Ned.-Indie 72: 114; Seidenf., 1992, Opera Bot.: 422.
Southern Cambodia, to the N of Sihanoukville town, Kaoh Rong Samloem Khong Island in Siam Gulf, 21 November 2011, T. Maisak, L. Osinovetz, M. Telepova, 721 (LE).

Epiphyte. Primary and secondary evergreen broad-leaved lowland forests on low hills, commonly in humid shady places along streams. Fl. JanuaryFebruary, May - June. Not common.

NE. India, Burma, S. China, Thailand, Vietnam, Laos, Malacca Peninsula, Sumatra, Java, Borneo, Moluccas, Philippines. Cambodia: Kampong Speu, Sihanoukville.

Schoenorchis Blume

${ }^{\mathrm{L}} \mathrm{S}$. scolopendria Aver.,

2012, Taiwania 57, 2: 134.

Fig. 10I.

Northeastern Laos, Houaphan province, Viengxay district, Xieng Luong village, 8 April 2015, $N$. T. Hiep, L. Averyanov, N. S. Khang et al., LA-VN 1174 (HNL, FOF, LE).

Creeping miniature epiphyte. Primary and secondary broad-leaved evergreen dry forests on rocky limestone, commonly on shady cliffs near hill and mountain tops, 600-700 m. Fl. May - June. Very rare. Ornamental.

NW. Vietnam. Laos: Houaphan.

${ }^{\text {L }}$ S. tixieri (Guillaumin) Seidenf.,

1975, Contrib. Revis. Orchid Fl. Cambodia. Laos Vietnam 102.

Fig. 16A, B.

Central Laos, Xiangkhouang province, Peak district, Khoang village, 3 April 2015, N. T. Hiep, L. Averyanov, N. S. Khang et al., LA-VN 993 / TM 1249 (HNL, FOF, LE).

Miniature epiphyte. Primary and secondary broad-leaved evergreen dry forests on rocky limestone, commonly on shady cliffs near hill and mountain tops, 1200-1450 m. Fl. May - June. Very rare. Ornamental.

SW. China (Yunnan), NW. Vietnam. Laos: Xiangkhouang.

\section{Smitinandia Holttum}

S. micrantha (Lindl.) Holttum,

1969, Gard. Bull. Singapore 25: 106; Newman et al., 2007, Checklist Vasc. Pl. Lao PDR: 280; Schuit. et al., 2008, Nord. Journ. Bot. 26: 309; Aver., 2013, Turczaninowia 16, 4: 42.

Central Laos, Xiangkhouang province, Ban Thathom town area, 26 October 2013, E. Konstantinov, $K-175$ (LE). 
Epiphyte. Primary and secondary broad-leaved evergreen forests on rocky limestone, commonly on hill and mountain tops, 200-600 m. Fl. January June, October. Occasional.

India, Bangladesh, Nepal, Thailand, Cambodia, Vietnam, Malacca Peninsula, Kalimantan. Laos: Bolikhamxai, Champasak, Khammouan, Louangphrabang, Vientiane, Xiangkhouang.

\section{Spathoglottis Blume}

\section{S. affinis de Vriese,}

1855, Ill. Orchid. Ind. Orient. 3, t. 15; Newman et al., 2007, Checklist Vasc. P1. Lao PDR: 281; Schuit. et al., 2008, Nord. Journ. Bot. 26: 309; Aver., 2013, Turczaninowia 16, 4: 42.

Fig. 16C, D.

Central Laos, Vientiane province, Phou Khao Khouay National Protected Area to about $40 \mathrm{~km} \mathrm{NE}$ of Vientiane, 9 December 2015, L. Averyanov, T. Maisak, AL 154b (LE - photo).

Terrestrial and lithophytic tuberiferous ephemeroid herb. Open grassy places in any kind of habitat, particularly along streams and rivers, coniferous woodlands, open secondary scrub along agricultural fields, pastures and roadsides, 100-1000 m. Fl. August - December. Common. Ornamental.

Myanmar, Thailand, Vietnam, Cambodia, Malacca Peninsula, Java. Laos: Bolikhamxai, Champasak, Vientiane.

\section{Sunipia Lindl.}

S. scariosa Lindl.,

1833, Gen. Sp. Orch. Pl.: 179; Newman et al., 2007, Checklist Vasc. Pl. Lao PDR: 281; Schuit. et al., 2008, Nord. Journ. Bot. 26: 310; Aver., 2013, Turczaninowia 16, 4: 42.

Northeastern Laos, Houaphan province, Xon district, Nong Hua Pa Noi village, 11 April 2015, N. T. Hiep, L. Averyanov, N. S. Khang et al., LA-VN 1232 (HNL, FOF, LE).

Creeping epiphyte. Primary and secondary broad-leaved evergreen forests on rocky limestone, commonly on hill and mountain tops, $1100-1750 \mathrm{~m}$. Fl. April - May. Occasional.

NE. India, Bhutan, Myanmar, S. China (Yunnan), Thailand, Vietnam. Laos: Houaphan, Louangphrabang, Vientiane, Xiangkhouang.

\section{Taeniophyllum Blume}

T. filiferum Aver., sp. nov.

Described from NE. Laos ("Houphan province, Viengxay district, Xieng Luong village, around point $20^{\circ} 22^{\prime} 06.1^{\prime \prime} \mathrm{N} 104^{\circ} 17^{\prime} 18.6$ ' $\mathrm{E}, 600-700 \mathrm{~m}$ a.s.l."). Type ("viscidium narrowly ovate, twice shorter.

Miniature canopy and branch epiphyte. Primary and old secondary lowland broad-leaved evergreen dry forests on rocky limestone, usually on old mossy trees in shady, humid places near mountain tops, 600-700 m. Fl. August - September. Rare.

Laos: Houphan. Endemic.

Species may be close to $T$. gracillimum Schltr. distributed in Thailand, Kalimantan and Malacca Peninsula and T. intermedium described from Malacca Peninsula, but distinctly differs in numerous fasciculate, rather leathery roots, numerous persistent scapes, few-flowered racemes with only 1-3(4) white flowers and very large apical appendage of the lip apex. New species in its habit and appearance somewhat resembles also T. fasciculatum Aver., known from southern Vietnam, but differs in fleshy roots and lip forming prominent apical seta.

\section{Tainia Blume}

${ }^{\mathrm{L}}$ T. cordifolia Hook.f., 1889, Icon. Pl. 19, t. 1861.

$\equiv$ Mischobulbum cordifolium (Hook. f.) Schltr., 1911, Repert. Spec. Nov. Regni Veg. Beih. 1: 98.

Fig. 16G-I.

Northeastern Laos, Houaphan province, Hem district, Nacoc village, 12 April 2015, N. T. Hiep, L. Averyanov, N. S. Khang et al., LA-VN 1377 (HNL, FOF, LE).

Terrestrial herb. Primary and secondary broadleaved evergreen forest, sometime bamboo thickets on rocky limestone, usually in shady depressions, 700-900 m. Fl. April - May. Rare. Ornamental.

SE. China, Hong Kong, Taiwan, Thailand, Vietnam, Malacca Peninsula, Kalimantan. Laos: Houaphan.

\section{Thelasis Blume}

${ }^{\mathrm{C}}$ T. pygmaea (Griff.) Lindl.,

1858, Journ. Proc. Linn. Soc., Bot. 3: 63; Schuit. et al., 2008, Nord. Journ. Bot. 26: 311; Aver., 2013, Turczaninowia 16, 4: 43.

Southern Cambodia, to the N of Sihanoukville town, Kaoh Rong Island in Siam Gulf, 19 November 2011, T. Maisak, M. Telepova, L. Osinovetz, 912 (LE). Northeastern Laos, Houaphan province, Hem district, Nacoc village, 12 April 2015, N. T. Hiep, L. Averyanov, N. S. Khang et al., LA-VN 1444 (HNL, FOF, LE).

Miniature epiphyte and occasionally lithophyte. Primary and secondary broad-leaved evergreen forests on any kind of soil, often on rocky limestone 
near mountain tops, 50-1200 m. Fl. April - August. Not rare.

India, Nepal, Myanmar, S. China, Thailand, Vietnam, Malacca Peninsula, Sumatra, Java, Kalimantan, Sulawesi, Lesser Sunda Islands, Philippines, New Guinea, tropical Pacific islands. Laos: Bolikhamxai, Houaphan, Khammouan, Vientiane. Cambodia: Sihanoukville.

Thrixspermum Lour.

${ }^{\mathrm{L}}$ T. calceolum (Lind1.) Rchb. f.,
1868, Xenia Orchid. 2: 122.

Northeastern Laos, Houaphan province, Viengxay district, Vieng Xai village, 7 April 2015, N. T. Hiep, L. Averyanov, N. S. Khang et al., LA-VN 1148 a (HNL, FOF, LE).

Epiphyte and lithophyte. Primary and secondary broad-leaved evergreen open forests on rocky limestone, commonly on hill and mountain tops, 800-950 m. Fl. April - July. Rare.

Thailand, Vietnam, Malacca Peninsula, Sumatra, Borneo. Laos: Houaphan.
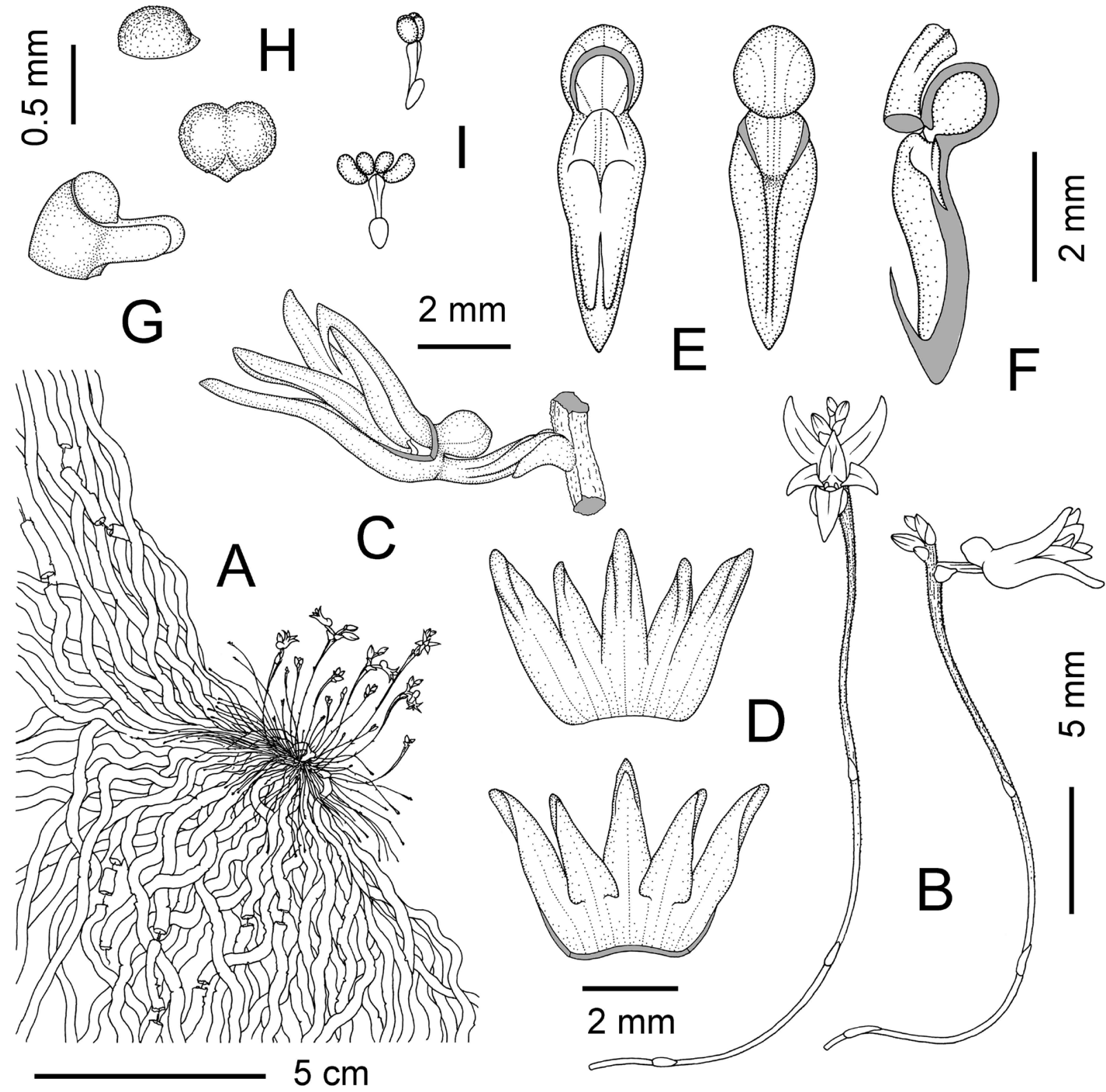

Fig. 15. New and rare orchids in the flora of Cambodia and Laos. Taeniophyllum filiferum Aver. A - Flowering plant. B - Inflorescences; C - Flower with lateral sepal and petal removed, side view. D - Flattened sepals and petals, abaxial and adaxial surface. E - lip, view from above and from below. F - Lip, sagittal section. G - Column, side view. $\mathrm{H}$ - Operculum. I - Pollinarium, frontal and side views. All drawn from the type specimen - LA-VN 1174b / TM 1273 by L. Averyanov. 

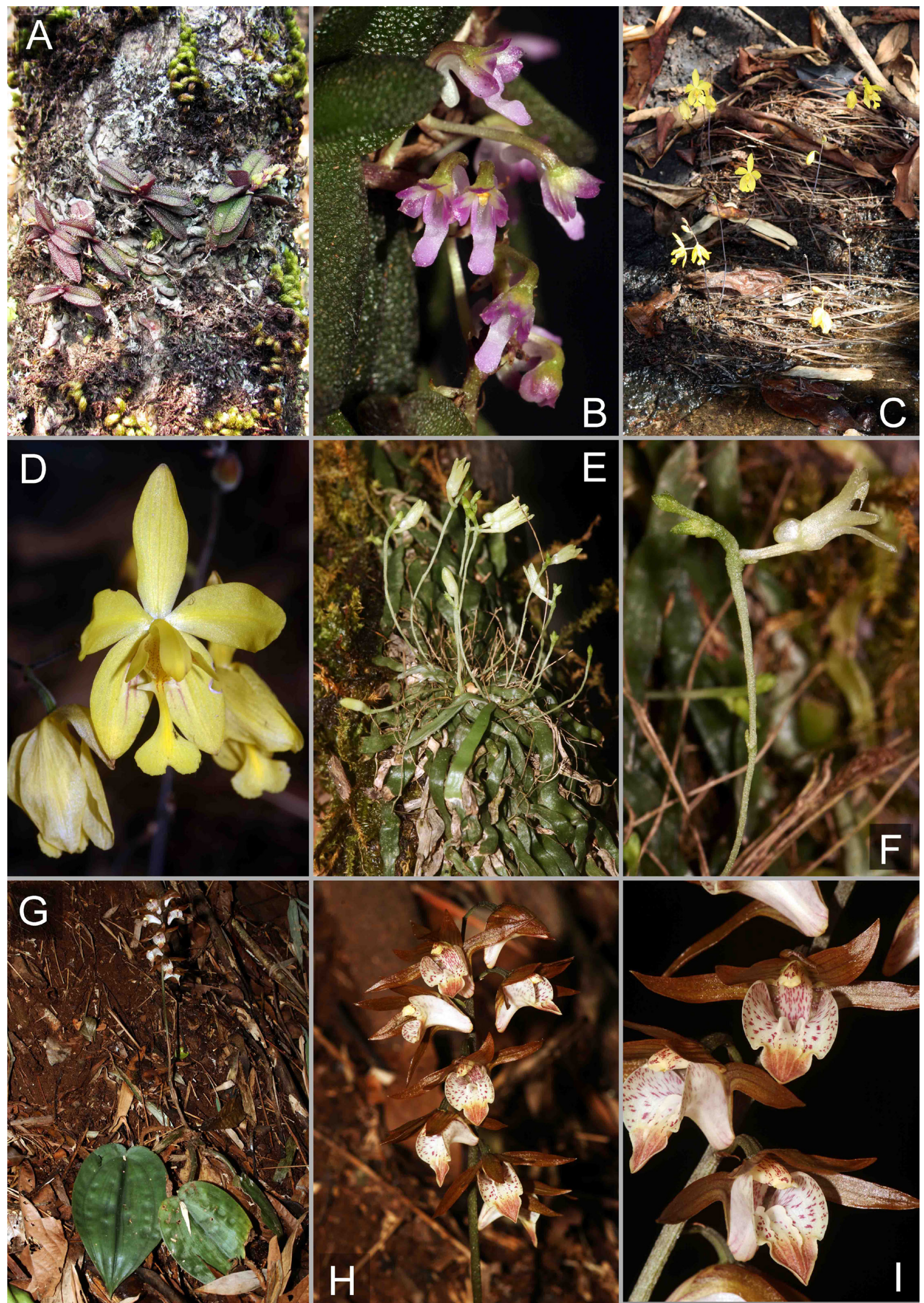

Fig. 16. New and rare orchids in the flora of Cambodia and Laos. A, B - Schoenorchis tixieri (Guillaumin) Seidenf. (LA-VN 993 / TM 1249). C, D - Spathoglottis affinis de Vriese (AL 154b). E, F - Taeniophyllum filiferum Aver. (LA-VN $1174 b$ / TM 1273). G-I - Tainia cordifolia Hook.f. (LA-VN 1377). Photos by K. S. Nguyen (A) and L. Averyanov (B-I). 
T. centipeda Lour.,

1790, F1. Cochinch. 520; Newman et al., 2007, Checklist Vasc. Pl. Lao PDR: 282; Schuit. et al., 2008, Nord. Journ. Bot. 26: 311; Aver., 2013, Turczaninowia 16, 4: 43.

$=T$. arachnites (Blume) Rchb. f., 1868, Xenia Orchid. 2: 121.

Central Laos, Vientiane province, Kasi town area, 25 October 2014, E. Konstantinov, K-292 (LE). Northeastern Laos, Houaphan province, Hem district, Khon Ngua village, 15 April 2015, N. T. Hiep, L. Averyanov, N. S. Khang et al., LA-VN 1501 (HNL, FOF, LE). Northeastern Laos, Houaphan province, Xon district, Nong Hua Pa Noi village, 11 April 2015, N. T. Hiep, L. Averyanov, N. S. Khang et al., LA-VN 1267 (HNL, FOF, LE).

Epiphyte. Primary and secondary broad-leaved and mixed evergreen and semideciduous forests on any kind of soil, preferably on rocky limestone, particularly along streams and on hill and mountain tops, 400-1200 m. Fl. May - June, September December. Not rare.

NE. India, Myanmar, S. China, Thailand, Vietnam, Cambodia, Malacca Peninsula, Sumatra, Java, Kalimantan, Philippines. Laos: Bolikhamxai, Champasak, Houaphan, Khammouan, Louang Namtha, Savannakhet, Vientiane.

Trias Lindl.

T. disciflora (Rolfe) Rolfe,

1896, Hand-List Orch. Cult. Roy. Gard. Kew: 35; Newman et al., 2007, Checklist Vasc. Pl. Lao PDR: 282; Schuit. et al., 2008, Nord. Journ. Bot. 26: 312.

Laos, Champasak province, Bolaven Plateau, Tad Gneuang Waterfall, 9 December 2014, E. Konstantinov, K-358 (LE).

Epiphyte. Primary and secondary broad-leaved and mixed evergreen and semideciduous forests, coniferous woodlands on any kind of soil, 500-1200 m. Fl. July - December. Not common.

NE. India, Thailand, Vietnam. Laos: Champasak, Khammouan, Vientiane.

\section{Trichoglottis Blume}

T. lorata (Downie) Schuit.,

2007, Orchideen Journ. 14: 62; Schuit. et al., 2008, Nord. Journ. Bot. 26: 312.

三Staurochilus loratus (Rolfe ex Downie) Seidenf., 1988, Opera Bot. 95: 95; Aver., 2015, Wulfenia 22: 182.

Northeastern Laos, Houaphan province, Hem district, Nacoc village, 12 April 2015, N. T. Hiep, L.
Averyanov, N. S. Khang et al., LA-VN 1410 (HNL, FOF, LE).

Epiphyte. Primary and secondary broad-leaved evergreen forests preferably on rocky limestone, 700-1250 m. Fl. April - June. Rare.

Thailand, Vietnam. Laos: Houaphan, Louang Namtha.

T. orchidea (J. Koenig) Garay,

1972, Bot. Mus. Leafl. 23: 209; Newman et al., 2007, Checklist Vasc. P1. Lao PDR: 282; Schuit. et al., 2008, Nord. Journ. Bot. 26: 313; Aver., 2013, Turczaninowia 16, 4: 43.

$\equiv$ Epidendrum orchideum J. Koenig, 1791, Observ. Bot. 6: 48.

= Trichoglottis cirrhifera Teijsm. et Binn., 1853, Natuurk. Tijdschr. Ned.-Indië 5: 493.

Northeastern Laos, Houaphan province, Hem district, Nacoc village, 12 April 2015, N. T. Hiep, L. Averyanov, N. S. Khang et al., LA-VN 1375 (HNL, FOF, LE). Northeastern Laos, Houaphan province, Hem district, Nacoc village, 12 April 2015, N. T. Hiep, L. Averyanov, N. S. Khang et al., LA-VN 1409 (HNL, FOF, LE).

Epiphyte. Primary and secondary broad-leaved evergreen forests preferably on rocky limestone, 200-1200 m. Fl. January - May. Occasional.

Thailand, Nicobar Islands, Malacca Peninsula, Java. Laos: Bolikhamxai, Champasak, Houaphan, Louangphrabang, Vientiane.

Trichotosia Blume

T. dasyphylla (Par. et Rchb.f.) Kraenzl.,

1911, Pflanzenr. IV, 50, 50: 138; Newman et al., 2007, Checklist Vasc. P1. Lao PDR: 282; Schuit. et al., 2008, Nord. Journ. Bot. 26: 313; Aver., 2013, Turczaninowia 16, 4: 43.

Northeastern Laos, Houaphan province, Xon district, Nong Hua Pa Noi village, 11 April 2015, N. T. Hiep, L. Averyanov, N. S. Khang et al., LA-VN 1236 (HNL, FOF, LE).

Creeping epiphyte. Primary and secondary broad-leaved and coniferous evergreen forests on any kind of soil, preferably on rocky limestone, 800-1350 m. Fl. April - July. Not rare.

NE. India, Nepal, Myanmar, Thailand, SW. China (Yunnan), Vietnam. Laos: Bolikhamxai, Houaphan, Khammouan, Louang Namtha, Saravan, Vientiane, Xaignabouri, Xiangkhouang.

T. pulvinata (Lindl.) Kraenzl.,

1911, Pflanzenr. IV, 50, 50: 138; Seidenf., 1992, Opera Bot. 114: 164. 
Southern Cambodia, to the $\mathrm{N}$ of Sihanoukville town, Kaoh Rong Samloem Khong Island in Siam Gulf, 21 November 2011, T. Maisak, L. Osinovetz, M. Telepova s.n. (LE).

Epiphyte. Primary and secondary evergreen broad-leaved lowland forests on low hills, commonly in shady humid places along streams. Fl. August September. Occasional.

NE. India, Myanmar, S. China, Thailand, Laos, Vietnam. Cambodia: Kampong Speu, Sihanoukville, "Kuang Krepeuh".

\section{Tropidia Lindl.}

\section{T. angulosa Blume,}

1859, Coll. Orchid.: 122; Schuit. et al., 2008, Nord. Journ. Bot. 26: 313; Aver., 2013, Turczaninowia 16, 4: 43.

Central Laos, Xiangkhouang province, Kham district, Huad village, 4 April 2015, N. T. Hiep, L. Averyanov, N. S. Khang et al., LA-VN 1078 (HNL, FOF, LE). Northeastern Laos, Houaphan province, Hem district, Khon Ngua village, 15 April 2015, N. T. Hiep, L. Averyanov, N. S. Khang et al., LA-VN 1469 (HNL, FOF, LE). Northeastern Laos, Houaphan province, Hem district, Nacoc village, 12 April 2015, N. T. Hiep, L. Averyanov, N. S. Khang et al., LA-VN 1411 (HNL, FOF, LE).

Terrestrial herb. Primary and secondary broadleaved evergreen forests on rocky limestone, commonly on shady slopes and in humid depressions, 200-1400 m. Fl. April - July. Not rare.

India, Bangladesh, Bhutan, Myanmar, S. China, Taiwan, Thailand, Vietnam, Malacca Peninsula, Sumatra, Java. Laos: Houaphan, Khammouan, Louang Namtha, Vientiane, Xiangkhouang.

\section{T. curculigoides Lindl.,}

1840, Gen. Sp. Orch. P1.: 497; Newman et al., 2007, Checklist Vasc. Pl. Lao PDR: 282; Schuit. et al., 2008, Nord. Journ. Bot. 26: 313; Aver., 2013, Turczaninowia 16, 4: 45.

Central Laos, Xiangkhouang province, Kham district, Huad village, 4 April 2015, N. T. Hiep, L. Averyanov, N. S. Khang et al., LA-VN 1070 (HNL, FOF, LE). Northeastern Laos, Houaphan province, Xon district, Nong Hua Pa Noi village, 11 April 2015, N. T. Hiep, L. Averyanov, N. S. Khang et al., $L A-V N 1276$ (HNL, FOF, LE).

Terrestrial herb. Primary and secondary broadleaved evergreen forests on rocky limestone, commonly on shady slopes and in humid depressions, 900-1400 m. Fl. April - July. Not rare.
India, Myanmar, China, Taiwan, Thailand, Vietnam, Cambodia, Malacca Peninsula, Java, Borneo, Lesser Sunda Islands, Australia, New Guinea. Laos: Bolikhamxai, Houaphan, Khammouan, Louangphrabang, Xaignabouri, Xiangkhouang.

Vanda $\mathrm{R}$. Br.

V. brunnea Rchb. f.,

1868, Xenia Orchid. 2: 138; Newman et al., 2007, Checklist Vasc. Pl. Lao PDR: 283; Schuit. et al., 2008, Nord. Journ. Bot. 26: 313; Aver., 2012, Taiwania 57, 2: 137; id., 2013, Turczaninowia 16, 4: 45.

Northeastern Laos, Houaphan province, Xon district, Nong Hua Pa Noi village, 11 April 2015, N. T. Hiep, L. Averyanov, N. S. Khang et al., LA-VN 1270 (HNL, FOF, LE).

Epiphyte. Primary and secondary broad-leaved and coniferous evergreen forests on any kind of soil preferably on rocky limestone, 1100-1400 m. Fl. March - May. Occasional. Ornamental.

Myanmar, Thailand, NW. Vietnam. Laos: Houaphan, Oudomxai, Phongsali.

V. denisoniana Benson et Rchb. f.,

1869, Gard. Chron. 1869: 528; Newman et al., 2007, Checklist Vasc. Pl. Lao PDR: 283; Schuit. et al., 2008, Nord. Journ. Bot. 26: 314.

Northeastern Laos, Houaphan province, Hem district, Na Puok village, 11 April 2015, N. T. Hiep, L. Averyanov, N. S. Khang et al., LA-VN 1325 (HNL, FOF, LE).

Epiphyte. Primary and secondary broad-leaved evergreen dry forests on any kind of soil, preferably on rocky limestone, (600)1000-1200 m. Fl. December - February. Not common. Ornamental.

Myanmar, S. China, Thailand, Vietnam. Laos: Champasak, Houaphan, Louangphrabang, Xiangkhouang.

\section{V. flavobrunnea Rchb. f.,}

1886, Flora 69: 552; Schuit. et al., 2008, Nord. Journ. Bot. 26: 314.

$=V$. pumila Hook.f., 1890, Fl. Brit. India 6: 53; Newman et al., 2007, Checklist Vasc. Pl. Lao PDR: 283.

Central Laos, Xiangkhouang province, Peak district, Khoang village, 3 April 2015, N. T. Hiep, L. Averyanov, N. S. Khang et al., LA-VN 971 (HNL, FOF, LE). Northeastern Laos, Houaphan province, 
Hem district, Na Puok village, 11 April 2015, N. T. Hiep, L. Averyanov, N. S. Khang et al., LA-VN 1303 (HNL, FOF, LE). Northeastern Laos, Houaphan province, Hem district, Nacoc village, 12 April 2015, N. T. Hiep, L. Averyanov, N. S. Khang et al., LA-VN 1454 (HNL, FOF, LE). Northeastern Laos, Houaphan province, Xon district, Nong Hua Pa Noi village, 11 April 2015, N. T. Hiep, L. Averyanov, N. S. Khang et al., LA-VN 1273 (HNL, FOF, LE).

Epiphyte. Primary and secondary broad-leaved and mixed evergreen forests on any kind of soil, preferably on rocky limestone, commonly on hill and mountain tops, 700-1450 m. Fl. March - May. Not common. Ornamental.

India, Nepal, Myanmar, SW. China (Yunnan), Thailand, Vietnam. Laos: Houaphan, Louang Namtha, Phongsali, Xiangkhouang.

\section{Acknowledgements}

Field works, results of which are presented in this paper were financially supported in part by U.S.A. National Geographic Society, grants "Flora of relict karstic formation of central Laos (Vientiane province, Vang Vieng and Kasi districts" (\# 9141-12), "Assessment of plant diversity in main limestone areas of central Laos" (\#9906-16) and Russian Foundation for Basic Research "Plant taxonomy, geography and biology in local floras of eastern Indochina" (15-04-00419A), and were carried out in the framework of institutional research project of the Komarov Botanical Institute of the Russian Academy of Sciences. The authors cordially thanks Dr. Philip Thomas and Mr. Robert Timmins for his data and photos on Calanthe lyroglossa and C. sylvatica used in this paper.

\section{REFERENCES}

Averyanov, L. V. (2012) New orchid taxa and records in the flora of Vietnam. Taiwania 57(2): 127-152.

Averyanov, L. V. (2013) New and rare orchids (Orchidaceae) in the flora of Cambodia and Lao PDR. Turczaninowia 16, 4: 26-46.

Averyanov, L. V., Averyanova A. L. (2003) Updated checklist of the orchids of Vietnam. Vietnam Nat. Univ. Publ. House, Hanoi, 102 pp.

Averyanov, L. V., Khang Sinh Nguyen, Nguyen Thien Tich, Phi Tam Nguyen, Van Duy Nong, Van Canh Nguyen, Canh Chu Xuan. (2015) New orchids in the flora of Vietnam. Wulfenia 22: 137-188.

Barretto, G., Cribb, P., Gale, S. (2011) The wild orchids of Hong Kong. Natural History Publications (Borneo), Kota Kinabalu, 697 pp.

Callaghan, M. (2004) Checklist of Lao Plant Names. Vientiane, 210 pp.

Gagnepain, F., Guillaumin, A. (1932-1934) Orchidaces and Apostasiaces. In: H. Lecomte (ed.). Flore Generale de l'Indochine 6. Masson et Cie, Paris, 142-652 pp.

Leti, M., Hul, S., Fouché, J.-G., Chéng, S. K., David, B. (2013) Flore Photographique du Cambodge. Editions Privat, France, 592 pp.

Newman, M. F., Ketphanh S., Svengsuksa B., Thomas P., Sengdala K., Lamxay V., Armstrong K. (2007) A checklist of the vascular plants of Lao PDR. Royal Botanic Garden, Edinburgh, 394 pp.

Orchids of Cambodia. URL: http://www.orchidcambodia.com (Accessed at 20 July 2013).

Pedersen, H. A., Kurzweil, H., Suddee, S., Cribb, P. J. (2011) Flora of Thailand. 12(1). Orchidaceae (Cypripedioideae, Orchidoideae, Vanilloideae). Forest Herb, Bangkok, 302 pp.

Schuiteman, A. (2016) Porpax verrucosa (Orchidaceae), a new species from Cambodia. Kew Bulletin 71: 1-5.

Schuiteman, A., Bonnet, P., Svengsuksa, B., Barthelemy, D. (2008) An annotated checklist of the Orchidaceae of Laos. Nordic Journal of Botany 26: 257-316.

Schuiteman, A., Ryani, C., Nut, M. (2015) New records of Orchidaceae from Cambodia I. Cambodian Journal of Natural History 2: 131-138.

Schuiteman, A., de Vogel, E. F. (2000) Orchid genera of Thailand, Laos, Cambodia and Vietnam. Nat. Herb. Nederlands, Leiden, 118 pp.

Seidenfaden, G. (1966) On a small collection of orchids from Laos. Nat. Hist. Bull. Siam. Soc. 21(1-2): 63-67.

Seidenfaden, G. (1973) An enumeration of Laotian orchids. Bull. Mus. Nat. Hist. (Paris), 3 ser., 71, Bot. Issue 5: 101-152.

Seidenfaden, G. (1975) Contributions to a revision of the Orchid flora of Cambodia, Laos, and Vietnam, 1. A preliminary enumeration of all orchids hitherto recorded. Kai Olsen, Fredensborg, 117 pp.

Seidenfaden, G. (1988) Orchid genera in Thailand 14. Fifty-nine vandoid genera. Opera Bot. 95: 1-398.

Seidenfaden, G. (1992) The orchids of Indochina. Opera Bot. 114: 1-502.

Svengsuksa, B., Lamxay, V. (2005) Field guide to the Orchids of Lao PDR. National University of Lao PDR, Vientiane, $185 \mathrm{pp}$. 
Tagane, S., Yukawa, T., Chhang, P., Ogura-Tsujita, Y., Toyama, H., Yahara, T. (2015) Anew record of Aphyllorchis pallida (Orchidaceae) from Cambodia. Cambodian Journal of Natural History 2: 128-130.

Vidal, J. (1959) Noms vernaculaires de plantes (Lao, Meo, Kha) en usage au Laos. Extrait du Bulletin de l'Ecole Francaise d'Etreme-Orient, Paris 49(2): 435-608. 